\title{
Homogeneous Molecular Systems are Positively Cooperative but Charged Molecular Systems are Negatively Cooperative
}

\author{
Chunying Rong, ${ }^{\dagger}$ Dongbo Zhao, ${ }^{\ddagger}$ Tianjing Zhou, ${ }^{\dagger}$ Siyuan Liu, ${ }^{\dagger}$ Donghai Yu, ${ }^{\dagger}$ and Shubin Liu,${ }^{*}$ \\ ${ }^{+}$College of Chemistry and Chemical Engineering, Hunan Normal University, \\ Changsha Hunan 410081, People's Republic of China \\ ${ }^{\ddagger}$ School of Chemistry and Chemical Engineering, Nanjing University, \\ Nanjing 210093, People's Republic of China \\ "Research Computing Center, University of North Carolina, \\ Chapel Hill, North Carolina 27599-3420, USA \\ *Corresponding Author. E-mail: shubin@email.unc.edu
}

\section{COMPUTATIONAL DETAILS}

All optimized molecular structures and electronic wave functions were obtained from the Gaussian $16^{1}$ package, version A03, with tight SCF convergence and ultrafine integration grids. Because of the different nature of interactions within these six systems studied in this work, different computational methods have been employed. The theory level for $(\mathrm{HF})_{n},\left(\mathrm{CO}_{2}\right)_{n}, \mathrm{Cl}_{2} \mathrm{Ar}_{n}, \mathrm{NH}_{3}\left(\mathrm{H}_{2} \mathrm{O}\right)_{n}, \mathrm{~F}^{-}\left(\mathrm{H}_{2} \mathrm{O}\right)_{n}$, and $\mathrm{Li}^{+}\left(\mathrm{H}_{2} \mathrm{O}\right)_{n}$ clusters is B3LYP/6-311+G(p,d), M062X/6-311+G(p,d), MP2/aug-cc-pVDZ, wB97XD/aug-cc-PVDZ, B3LYP/aug-cc$\mathrm{pVDZ}$, and M062X/6-311+G(d,p), respectively. ${ }^{2-5}$ For all the systems studied in this work, it is required that the optimized structure for a given $n$ (i.e., the number of building blocks) be the global minimum. To make this happen, we either obtained the starting structures from the literature and then re-optimized with our chosen method, or performed molecular dynamics simulations to sample as many local minima as possible before the lowest-energy structure was picked as the representation of the global minimum. It does not guaranty that the cooperativity profile is smooth, however, because there exists the so-called odd-even phenomenon for molecular clusters, where, even for the global minimum, the energy profile fluctuates with respect to the odd or even numbers of the building blocks. This might be the reason why sometimes we see oscillations in the energy profile, which could be indications of the existence of either local minima or odd-even number fluctuation. Notice that, for a given system, what matters is the general trend, so even if oscillations are present, they should not qualitatively invalidate our main conclusions because the general trend is always apparent. A full structure optimization was followed by a single-point frequency calculation to ensure that the final optimized structure has no imaginary frequency. This frequency calculation also obtains the thermochemistry quantities needed to correlate with the 
interaction energy. The Multiwfn 3.4.1 program $^{6}$ was utilized to calculate the information-theoretic quantities aforementioned by using the checkpoint file from the Gaussian calculations as the input file. The energy components were obtained from Gaussian calculations with the keyword of iop $(5 / 33=1)$. The steric energy component was obtained via Fisher information, which differs from the Weizsäcker kinetic energy by a factor of 8 . To obtain the electron density for the isolated state used in evaluating information gain and other relative entropy quantities, we employed the spherically-averaged electron density of the neutral atom at the same level of theory. To perform the atomic partition, we choose the Hirshfeld's stockholder approach to partition atoms in molecules to obtain atomic values. ${ }^{7}$

\section{CARTESIAN COORDINATES OF ALL OPTIIMIZED STRUCTURES STUDIED IN THIS WORK}

(a) $(\mathrm{HF})_{n}$ systems, B3LYP/6-311+G(p,d)

System (HF)1

$\begin{array}{llll}\mathrm{F} & 0.00000 & 0.00000 & 0.09222 \\ \mathrm{H} & 0.00000 & 0.00000 & -0.82999\end{array}$

System (HF)2

$\begin{array}{lrrr}\text { F } & 1.44033 & 0.02366 & -0.00000 \\ \text { H } & 0.52665 & -0.14384 & 0.00001 \\ \text { F } & -1.30461 & -0.08813 & -0.00000 \\ \text { H } & -1.74812 & 0.72406 & -0.00000\end{array}$

System (HF)3

$\begin{array}{llll}\text { F } & -1.50313 & -0.15827 & -0.00001 \\ \text { H } & -0.87015 & -0.85313 & -0.00000 \\ \text { F } & 0.88863 & -1.22260 & -0.00007 \\ \text { H } & 1.17390 & -0.32699 & 0.00003 \\ \text { F } & 0.61450 & 1.38087 & 0.00007 \\ \text { H } & -0.30375 & 1.18014 & 0.00009\end{array}$

System (HF)4

$\begin{array}{llll}\text { F } & -1.24604 & -1.29822 & 0.00005 \\ \text { H } & -0.31139 & -1.47285 & 0.00016 \\ \text { F } & 1.29824 & -1.24603 & 0.00007 \\ \text { H } & 1.47286 & -0.31138 & 0.00007 \\ \text { F } & 1.24604 & 1.29823 & -0.00005 \\ \text { H } & 0.31139 & 1.47285 & -0.00016 \\ \text { F } & -1.29824 & 1.24602 & -0.00007 \\ \text { H } & -1.47287 & 0.31137 & -0.00006\end{array}$

System (HF)5

$\begin{array}{cccc}\mathrm{F} & 2.06411 & 0.55349 & 0.00002 \\ \mathrm{H} & 1.81280 & -0.36840 & 0.00000 \\ \mathrm{~F} & 1.16423 & -1.79199 & -0.00004\end{array}$




$\begin{array}{llll}\mathrm{H} & 0.20980 & -1.83782 & 0.00001 \\ \mathrm{~F} & -1.34455 & -1.66098 & 0.00005 \\ \mathrm{H} & -1.68310 & -0.76744 & 0.00003 \\ \mathrm{~F} & -1.99524 & 0.76547 & -0.00003 \\ \mathrm{H} & -1.25002 & 1.36353 & -0.00001 \\ \mathrm{~F} & 0.11145 & 2.13402 & -0.00000 \\ \mathrm{H} & 0.91054 & 1.61011 & 0.00001\end{array}$

System (HF)6

$\begin{array}{llll}\text { F } & -0.06709 & -2.49882 & -0.01036 \\ \text { H } & -0.90040 & -2.02921 & -0.00255 \\ \text { F } & -2.19809 & -1.19150 & 0.01048 \\ \text { H } & -2.20831 & -0.23504 & 0.00283 \\ \text { F } & -2.13098 & 1.30768 & -0.01049 \\ \text { H } & -1.30755 & 1.79439 & -0.00281 \\ \text { F } & 0.06708 & 2.49882 & 0.01037 \\ \text { H } & 0.90040 & 2.02922 & 0.00278 \\ \text { F } & 2.19809 & 1.19150 & -0.01029 \\ \text { H } & 2.20830 & 0.23504 & -0.00278 \\ \text { F } & 2.13099 & -1.30768 & 0.01027 \\ \text { H } & 1.30755 & -1.79439 & 0.00270\end{array}$

System (HF)7

$\begin{array}{lccc}\text { F } & 1.70355 & 2.13344 & -0.38147 \\ \text { H } & 0.83216 & 2.33060 & -0.03799 \\ \text { F } & -0.57515 & 2.54093 & 0.54873 \\ \text { H } & -1.35172 & 2.06836 & 0.24789 \\ \text { F } & -2.55015 & -1.24533 & -0.29191 \\ \text { H } & -1.81311 & -1.77022 & 0.01950 \\ \text { F } & -0.58756 & -2.53667 & 0.55266 \\ \text { H } & 0.30023 & -2.42040 & 0.21309 \\ \text { F } & 1.69808 & -2.14043 & -0.36106 \\ \text { H } & 2.18066 & -1.33754 & -0.16162 \\ \text { F } & 2.86240 & -0.00348 & 0.18395 \\ \text { H } & 2.45445 & 0.83774 & -0.02185 \\ \text { F } & -2.55385 & 1.25122 & -0.25096 \\ \text { H } & -2.57852 & 0.29438 & -0.25861\end{array}$

System (HF)8

$\begin{array}{llll}\text { F } & 3.04084 & 1.27573 & -0.24138 \\ \text { H } & 2.29532 & 1.78629 & 0.07376 \\ \text { F } & 1.06064 & 2.52485 & 0.62141 \\ \text { H } & 0.21698 & 2.56056 & 0.16948 \\ \text { F } & -3.07620 & 1.21801 & 0.19518 \\ \text { H } & -3.08567 & 0.26043 & 0.20418 \\ \text { F } & -3.04085 & -1.27573 & 0.24134 \\ \text { H } & -2.29532 & -1.78629 & -0.07380 \\ \text { F } & 1.11231 & -2.52762 & 0.59354\end{array}$




$\begin{array}{llll}\mathrm{H} & 1.88274 & -2.04087 & 0.29684 \\ \mathrm{~F} & 3.07621 & -1.21801 & -0.19514 \\ \mathrm{H} & 3.08567 & -0.26043 & -0.20416 \\ \mathrm{~F} & -1.11231 & 2.52763 & -0.59353 \\ \mathrm{H} & -1.88274 & 2.04088 & -0.29682 \\ \mathrm{~F} & -1.06064 & -2.52486 & -0.62142 \\ \mathrm{H} & -0.21698 & -2.56056 & -0.16948\end{array}$

System (HF) 9

$\begin{array}{llll}\text { F } & -2.69203 & -2.17290 & 0.36800 \\ \text { H } & -3.02096 & -1.34392 & 0.01757 \\ \text { F } & -3.47339 & -0.00777 & -0.57920 \\ \text { H } & -3.19741 & 0.84056 & -0.22943 \\ \text { F } & -0.55444 & 3.17495 & -0.39333 \\ \text { H } & 0.28706 & 2.97346 & 0.01743 \\ \text { F } & 3.38020 & 1.24741 & -0.39184 \\ \text { H } & 3.39942 & 0.28966 & -0.39415 \\ \text { F } & 1.60281 & -2.55483 & 0.71849 \\ \text { H } & 0.77669 & -2.82004 & 0.31135 \\ \text { F } & -0.54082 & -3.17373 & -0.37977 \\ \text { H } & -1.38398 & -2.80923 & -0.10568 \\ \text { F } & -2.69803 & 2.15986 & 0.36786 \\ \text { H } & -1.88130 & 2.57721 & 0.09148 \\ \text { F } & 3.38664 & -1.24476 & -0.42636 \\ \text { H } & 2.71365 & -1.77835 & -0.00344 \\ \text { F } & 1.59052 & 2.57163 & 0.71582 \\ \text { H } & 2.29380 & 2.07208 & 0.29792\end{array}$

System (HF)10

$\begin{array}{llll}\text { F } & 2.88006 & 1.79852 & 0.81283 \\ \text { H } & 3.34416 & 1.08191 & 0.37681 \\ \text { F } & 4.04565 & -0.06718 & -0.34393 \\ \text { H } & 3.72144 & -0.96869 & -0.34881 \\ \text { F } & 0.97561 & -2.97454 & 0.64763 \\ \text { H } & 0.14165 & -3.00128 & 0.17638 \\ \text { F } & -4.05286 & 0.15967 & 0.36230 \\ \text { H } & -3.56938 & 0.86069 & -0.07537 \\ \text { F } & -1.09972 & 3.40232 & 0.30952 \\ \text { H } & -0.16837 & 3.40673 & 0.08322 \\ \text { F } & 1.30767 & 3.35999 & -0.31880 \\ \text { H } & 1.94103 & 2.77693 & 0.10139 \\ \text { F } & 3.17092 & -2.40007 & -0.38251 \\ \text { H } & 2.32826 & -2.65198 & -0.00469 \\ \text { F } & -2.73975 & 1.91485 & -0.81958 \\ \text { H } & -2.11506 & 2.50888 & -0.40007 \\ \text { F } & -3.32451 & -2.22276 & 0.35117 \\ \text { H } & -3.61768 & -1.31062 & 0.34391 \\ \text { F } & -1.16321 & -2.97189 & -0.61861\end{array}$




\begin{tabular}{|c|c|c|c|}
\hline $\mathrm{H}$ & -2.00460 & -2.69274 & -0.25288 \\
\hline \multicolumn{4}{|c|}{ System (HF)11 } \\
\hline $\mathrm{F}$ & 2.64768 & 2.48091 & 0.71689 \\
\hline $\mathrm{H}$ & 3.27495 & 1.91321 & 0.26565 \\
\hline $\mathrm{F}$ & 4.24437 & 0.99983 & -0.47828 \\
\hline $\mathrm{H}$ & 4.20140 & 0.04285 & -0.45440 \\
\hline $\mathrm{F}$ & 2.27172 & -2.66413 & 0.75704 \\
\hline $\mathrm{H}$ & 1.53979 & -3.12283 & 0.34074 \\
\hline $\mathrm{F}$ & -3.55769 & -1.97845 & -0.60416 \\
\hline $\mathrm{H}$ & -3.79479 & -1.12913 & -0.22826 \\
\hline $\mathrm{F}$ & -1.50968 & 3.69955 & 0.50362 \\
\hline $\mathrm{H}$ & -0.61042 & 3.73132 & 0.17409 \\
\hline $\mathrm{F}$ & 0.80960 & 3.73152 & -0.39715 \\
\hline $\mathrm{H}$ & 1.54024 & 3.26908 & 0.01563 \\
\hline$F$ & 4.10694 & -1.48684 & -0.44440 \\
\hline $\mathrm{H}$ & 3.41157 & -1.97189 & 0.00058 \\
\hline $\mathrm{F}$ & -3.30542 & 2.35463 & -0.56888 \\
\hline $\mathrm{H}$ & -2.61901 & 2.89249 & -0.17095 \\
\hline$F$ & -1.95939 & -3.55489 & 0.46546 \\
\hline $\mathrm{H}$ & -2.59581 & -2.95739 & 0.06961 \\
\hline $\mathrm{F}$ & 0.37012 & -3.80194 & -0.36473 \\
\hline $\mathrm{H}$ & -0.53584 & -3.72381 & -0.06037 \\
\hline $\mathrm{F}$ & -4.11718 & 0.21976 & 0.41411 \\
\hline $\mathrm{H}$ & -3.82167 & 1.05662 & 0.05203 \\
\hline
\end{tabular}

\begin{tabular}{|c|c|c|c|}
\hline \multicolumn{4}{|c|}{ yystem (HF)12 } \\
\hline $\mathrm{F}$ & 2.01110 & -2.65526 & 0.87557 \\
\hline $\mathrm{H}$ & 1.36425 & -3.23579 & 0.46916 \\
\hline $\mathrm{F}$ & 0.33189 & -4.12067 & -0.21339 \\
\hline $\mathrm{H}$ & -0.60405 & -4.06022 & -0.01295 \\
\hline F & -3.57454 & -2.34476 & -0.85228 \\
\hline $\mathrm{H}$ & -3.95429 & 990 & 5361 \\
\hline 1 & -2.34483 & & 249 \\
\hline $\mathrm{H}$ & -1.54138 & 3.80491 & 0.63578 \\
\hline $\mathrm{F}$ & 3.56261 & 2.23 & 0.3 \\
\hline $\mathrm{H}$ & 4.07323 & 57 & 0.03694 \\
\hline $\mathrm{F}$ & 4.85886 & 0.28690 & -0.48806 \\
\hline $\mathrm{H}$ & 4.63810 & -0.6 & 938 \\
\hline $\mathrm{F}$ & -2.08017 & -3.9 & 139 \\
\hline $\mathrm{H}$ & -2.67909 & -3.3 & -0.09805 \\
\hline $\mathrm{F}$ & 1.57222 & 3.10 & -0.85853 \\
\hline $\mathrm{H}$ & 2.35490 & 2.77991 & -0.40854 \\
\hline $\mathrm{F}$ & -3.83254 & 1.97389 & -0.46590 \\
\hline $\mathrm{H}$ & -3.26838 & 2.54005 & 0.06292 \\
\hline $\mathrm{F}$ & -4.51746 & -0.31457 & 0.22807 \\
\hline $\mathrm{H}$ & -4.27104 & & -0.02330 \\
\hline 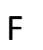 & -0.25086 & 4.46581 & 0.15015 \\
\hline
\end{tabular}




$\begin{array}{llll}\mathrm{H} & 0.47698 & 3.96701 & -0.22160 \\ \mathrm{~F} & 4.26429 & -2.08575 & -0.01592 \\ \mathrm{H} & 3.40567 & -2.34811 & 0.31618\end{array}$

System (HF)13

$\begin{array}{llll}\text { F } & -4.63844 & -0.94405 & -0.32064 \\ \text { H } & -4.21589 & -1.65307 & 0.16636 \\ \text { F } & -3.49784 & -2.72837 & 0.98007 \\ \text { H } & -2.94021 & -3.41218 & 0.60456 \\ \text { F } & 0.16967 & -3.89275 & -0.96764 \\ \text { H } & 1.01898 & -3.94441 & -0.52483 \\ \text { F } & 4.64723 & -0.35400 & 0.76978 \\ \text { H } & 4.54824 & 0.50973 & 0.36551 \\ \text { F } & -1.41246 & 4.38202 & 0.36086 \\ \text { H } & -2.14956 & 3.92580 & -0.04852 \\ \text { F } & -3.28017 & 3.16822 & -0.74112 \\ \text { H } & -3.81130 & 2.49776 & -0.30799 \\ \text { F } & -2.03961 & -4.49132 & 0.00773 \\ \text { H } & -1.17463 & -4.29574 & -0.35406 \\ \text { F } & 2.86561 & 3.61033 & 0.61436 \\ \text { H } & 2.09592 & 3.95594 & 0.15913 \\ \text { F } & 4.22692 & -2.49861 & -0.41495 \\ \text { H } & 4.41201 & -1.66798 & 0.02621 \\ \text { F } & 2.34638 & -3.99311 & 0.21998 \\ \text { H } & 3.08445 & -3.42445 & -0.00747 \\ \text { F } & 4.35525 & 1.85708 & -0.32554 \\ \text { H } & 3.78921 & 2.54941 & 0.02008 \\ \text { F } & -4.61732 & 1.42844 & 0.42563 \\ \text { H } & -4.64171 & 0.50954 & 0.15415 \\ \text { F } & 0.87475 & 4.45693 & -0.60857 \\ \text { H } & -0.01539 & 4.44228 & -0.25284\end{array}$

\begin{tabular}{lccc}
\multicolumn{4}{l}{ System (HF)14 } \\
F & -2.82082 & -4.33038 & -0.15869 \\
H & -1.91150 & -4.12984 & -0.38225 \\
F & -0.49049 & -3.70493 & -0.76378 \\
H & 0.31826 & -4.05049 & -0.38003 \\
F & 3.80301 & -3.59280 & -0.31147 \\
H & 4.18129 & -2.83698 & 0.14070 \\
F & 2.49580 & 3.71199 & -0.99292 \\
H & 1.76375 & 4.23213 & -0.65612 \\
F & -5.08012 & 1.68808 & 0.41684 \\
H & -5.10557 & 0.80353 & 0.04872 \\
F & -5.09477 & -0.59098 & -0.57787 \\
H & -4.83796 & -1.38245 & -0.10191 \\
F & 1.59334 & -4.57375 & 0.26213 \\
H & 2.45482 & -4.20428 & 0.05914 \\
F & -1.40312 & 3.93762 & 0.85428
\end{tabular}




$\begin{array}{lccc}\text { H } & -2.22970 & 3.76025 & 0.40119 \\ \text { F } & 4.27796 & 2.67379 & 0.39250 \\ \text { H } & 3.59717 & 3.10282 & -0.12803 \\ \text { F } & 4.71926 & -1.62459 & 0.89853 \\ \text { H } & 4.96017 & -0.79767 & 0.47696 \\ \text { F } & 0.59521 & 5.05305 & -0.11982 \\ \text { H } & -0.19581 & 4.64938 & 0.23945 \\ \text { F } & -4.39327 & -2.60395 & 0.69819 \\ \text { H } & -3.78994 & -3.27214 & 0.36847 \\ \text { F } & -3.51407 & 3.45463 & -0.35802 \\ \text { H } & -4.13012 & 2.77535 & -0.07731 \\ \text { F } & 5.31209 & 0.50237 & -0.23963 \\ \text { H } & 4.92491 & 1.34903 & -0.01123\end{array}$

System (HF) 15

$\begin{array}{lccc}\text { F } & -0.45401 & -5.49226 & -0.37561 \\ \text { H } & 0.35158 & -5.34286 & 0.12197 \\ \text { F } & 1.60072 & -5.06729 & 0.95892 \\ \text { H } & 2.37636 & -4.60512 & 0.63722 \\ \text { F } & 5.57658 & 0.08875 & 0.01954 \\ \text { H } & 5.09895 & 0.79447 & -0.41833 \\ \text { F } & -0.63123 & 5.51479 & 0.32628 \\ \text { H } & -1.20057 & 4.79397 & 0.59735 \\ \text { F } & -5.37188 & -1.03827 & -0.76649 \\ \text { H } & -4.94790 & -1.87873 & -0.58502 \\ \text { F } & -4.25722 & -3.20271 & -0.25898 \\ \text { H } & -3.37411 & -3.41046 & -0.56684 \\ \text { F } & 3.58028 & -3.84999 & 0.07555 \\ \text { H } & 3.93552 & -3.05894 & 0.48412 \\ \text { F } & -4.32419 & 3.05816 & 0.27777 \\ \text { H } & -4.74956 & 2.22027 & 0.46937 \\ \text { F } & 1.46417 & 5.15079 & -0.96347 \\ \text { H } & 0.65644 & 5.29416 & -0.46733 \\ \text { F } & 4.28155 & 1.85987 & -1.15196 \\ \text { H } & 3.96974 & 2.67881 & -0.76280 \\ \text { F } & -2.02156 & 3.57931 & 1.03876 \\ \text { H } & -2.91774 & 3.38086 & 0.75940 \\ \text { F } & -1.95000 & -3.64037 & -1.07276 \\ \text { H } & -1.37457 & -4.35908 & -0.80239 \\ \text { F } & -5.38276 & 0.87608 & 0.81746 \\ \text { H } & -5.39480 & 0.12553 & 0.22108 \\ \text { F } & 3.45990 & 3.95268 & -0.09663 \\ \text { H } & 2.69335 & 4.43264 & -0.41513 \\ \text { F } & 4.42998 & -1.78952 & 1.17172 \\ \text { H } & 4.87438 & -1.06552 & 0.72640\end{array}$

System (HF)16

$\begin{array}{llll}F & -0.01490 & -0.45169 & 2.86116\end{array}$ 


$\begin{array}{lccc}\text { H } & 0.81640 & -0.56111 & 2.39993 \\ \text { F } & 2.20603 & 3.41204 & 0.22454 \\ \text { H } & 1.36831 & 3.25147 & -0.20501 \\ \text { F } & -2.16744 & 3.42018 & 0.24272 \\ \text { H } & -2.68223 & 2.70497 & 0.61866 \\ \text { F } & -3.41300 & 1.53074 & 1.30546 \\ \text { H } & -2.96039 & 0.69894 & 1.42905 \\ \text { F } & -2.12799 & -0.61279 & 1.56772 \\ \text { H } & -1.31695 & -0.61048 & 2.08194 \\ \text { F } & -0.00459 & 2.87527 & -0.83453 \\ \text { H } & -0.85246 & 3.10602 & -0.44359 \\ \text { F } & 3.40219 & 1.50680 & 1.30279 \\ \text { H } & 2.97923 & 2.24370 & 0.85807 \\ \text { F } & 2.12033 & -0.59496 & 1.56596 \\ \text { H } & 2.64577 & 0.20586 & 1.47436 \\ \text { F } & 2.12015 & 0.59497 & -1.56598 \\ \text { H } & 2.64562 & -0.20585 & -1.47445 \\ \text { F } & -0.01510 & 0.45169 & -2.86116 \\ \text { H } & 0.81621 & 0.56112 & -2.39995 \\ \text { F } & -2.12814 & 0.61278 & -1.56764 \\ \text { H } & -1.31713 & 0.61047 & -2.08190 \\ \text { F } & -3.41311 & -1.53075 & -1.30523 \\ \text { H } & -2.96053 & -0.69895 & -1.42889 \\ \text { F } & -2.16742 & -3.42019 & -0.24264 \\ \text { H } & -2.68225 & -2.70498 & -0.61853 \\ \text { F } & -0.00453 & -2.87526 & 0.83451 \\ \text { H } & -0.85242 & -3.10601 & 0.44361 \\ \text { F } & 2.20603 & -3.41204 & -0.22468 \\ \text { H } & 1.36833 & -3.25147 & 0.20492 \\ \text { F } & 3.40208 & -1.50677 & -1.30298 \\ \text { H } & 2.97918 & -2.24370 & -0.85825\end{array}$

\begin{tabular}{lccc}
\multicolumn{4}{c}{ System (HF)17 } \\
F & 3.76937 & -1.44491 & -1.22239 \\
H & 3.00180 & -1.97161 & -1.44437 \\
F & -0.00855 & 3.11040 & -1.87600 \\
H & 0.75923 & 2.87096 & -1.35808 \\
F & 1.71962 & -2.71687 & -1.88843 \\
H & 0.88493 & -2.60972 & -1.43817 \\
F & -2.54764 & -1.99686 & -2.10855 \\
H & -2.85165 & -1.10179 & -2.27327 \\
F & -3.23159 & 0.35043 & -2.58514 \\
H & -2.87516 & 1.06933 & -2.06591 \\
F & -2.21375 & 2.18092 & -1.20704 \\
H & -1.37684 & 2.58160 & -1.45347 \\
F & -0.50017 & -2.33605 & -0.76392 \\
H & -1.30069 & -2.22899 & -1.28705 \\
F & 3.73206 & 1.01379 & -1.61813
\end{tabular}




\begin{tabular}{|c|c|c|c|}
\hline $\mathrm{H}$ & 3.78451 & 0.06848 & -1.46505 \\
\hline & 1.95028 & 2.34020 & -0.52295 \\
\hline & 2.67128 & 1.84573 & -0.92360 \\
\hline & 1.81046 & -1.95966 & 1.22015 \\
\hline & 2.23319 & -1.12355 & 1.42515 \\
\hline & -0.15104 & -2.86873 & 2.49602 \\
\hline & 0.60415 & -2.54371 & 2.01040 \\
\hline & -2.51134 & -2.46424 & 1.78736 \\
\hline & -1.60482 & -2.65974 & 2.02741 \\
\hline & -2.99265 & -0.31350 & 0.65568 \\
\hline & -2.83864 & -1.15595 & 1.09249 \\
\hline & -2.63895 & 1.81274 & 1.93787 \\
\hline & -2.83772 & 1.02328 & 1.43722 \\
\hline & -0.49975 & 2.95763 & 1.38850 \\
\hline & -1.35548 & 2.56882 & 1.58327 \\
\hline & 1.46764 & 2.06590 & 2.65180 \\
\hline & 0.74267 & 2.45707 & 2.16426 \\
\hline & 2.84775 & 0.26004 & 1.64949 \\
\hline & 2.34350 & 0.98881 & 2.01984 \\
\hline \multicolumn{4}{|c|}{ System (HF)18 } \\
\hline & 3.62658 & 2.77404 & 0.46040 \\
\hline & 2.83187 & 3.25870 & 0.23478 \\
\hline & -0.43722 & -0.51352 & 2.61833 \\
\hline & 0.37454 & -0.74766 & 2.16957 \\
\hline & 1.54643 & 4.06326 & -0.03744 \\
\hline & 0.72164 & 3.66007 & -0.30046 \\
\hline & -2.76469 & 3.41192 & 0.42667 \\
\hline 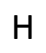 & -3.25802 & 2.66947 & 0.77882 \\
\hline & -3.95299 & 1.44849 & 1.41315 \\
\hline t & -3.48711 & 0.61609 & 1.46217 \\
\hline & -2.64055 & -0.69300 & 1.48618 \\
\hline & -1.78919 & -0.68980 & 1.92930 \\
\hline & -0.60476 & 2.94701 & -0.68431 \\
\hline & -1.45312 & 3.14518 & -0.27473 \\
\hline & 3.52870 & 0.65310 & 1.73653 \\
\hline t & 3.5 & 1.48070 & 1.24965 \\
\hline & 1.70459 & -0.98309 & 1.40782 \\
\hline & 2.42679 & -0.36200 & 1.54320 \\
\hline 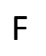 & 1.70451 & 0.98312 & -1.40780 \\
\hline 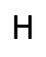 & 2.42674 & 0.36207 & -1.54320 \\
\hline & -0.43728 & 0.51351 & -2.61833 \\
\hline $\mathrm{H}$ & 0.37447 & 0.74766 & -2.16955 \\
\hline$r$ & -2.64063 & 0.69293 & -1.48620 \\
\hline $\mathrm{H}$ & -1.78927 & 0.68976 & -1.92931 \\
\hline $\mathrm{F}$ & -3.95296 & -1.44863 & -1.41311 \\
\hline 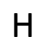 & -3.48713 & -0.61621 & -1.46215 \\
\hline & -2.76456 & -3.41201 & -0.4266 \\
\hline
\end{tabular}




$\begin{array}{llll}\mathrm{H} & -3.25793 & -2.66958 & -0.77878 \\ \mathrm{~F} & -0.60463 & -2.94701 & 0.68432 \\ \mathrm{H} & -1.45299 & -3.14520 & 0.27476 \\ \mathrm{~F} & 1.54658 & -4.06321 & 0.03742 \\ \mathrm{H} & 0.72178 & -3.66005 & 0.30045 \\ \mathrm{~F} & 3.62669 & -2.77393 & -0.46045 \\ \mathrm{H} & 2.83200 & -3.25861 & -0.23482 \\ \mathrm{~F} & 3.52870 & -0.65298 & -1.73656 \\ \mathrm{H} & 3.57619 & -1.48058 & -1.24969\end{array}$

System (HF)19

$\begin{array}{lccc}\text { F } & -4.18430 & -0.37645 & 1.76891 \\ \text { H } & -3.37457 & -0.86678 & 1.64292 \\ \text { F } & 3.01859 & 3.65259 & 0.66189 \\ \text { H } & 2.17116 & 3.89336 & 0.28687 \\ \text { F } & -0.00602 & -0.90284 & 2.57961 \\ \text { H } & 0.83490 & -0.82599 & 2.12968 \\ \text { F } & 0.78872 & 4.32892 & -0.24488 \\ \text { H } & 0.10814 & 3.70891 & -0.49514 \\ \text { F } & -3.39767 & 3.18113 & -0.56766 \\ \text { H } & -4.01769 & 2.51410 & -0.26265 \\ \text { F } & -4.99137 & 1.47250 & 0.28151 \\ \text { H } & -4.68964 & 0.75080 & 0.83066 \\ \text { F } & -2.04685 & -1.63129 & 1.37576 \\ \text { H } & -1.23860 & -1.39977 & 1.84102 \\ \text { F } & -1.00185 & 2.65740 & -0.82225 \\ \text { H } & -1.94060 & 2.86167 & -0.74278 \\ \text { F } & 3.37289 & 1.53562 & 1.90210 \\ \text { H } & 3.23466 & 2.35799 & 1.42413 \\ \text { F } & 2.16598 & -0.55948 & 1.38286 \\ \text { H } & 2.64957 & 0.24638 & 1.58860 \\ \text { F } & 1.78954 & 1.38998 & -1.17935 \\ \text { H } & 2.65453 & 1.01339 & -1.37710 \\ \text { F } & -0.10404 & -0.18413 & -1.57593 \\ \text { H } & 0.58166 & 0.46909 & -1.43124 \\ \text { F } & -2.56198 & 0.01371 & -1.28696 \\ \text { H } & -1.61152 & 0.06278 & -1.39715 \\ \text { F } & -3.28505 & -2.39879 & -1.44661 \\ \text { H } & -3.07448 & -1.46908 & -1.40822 \\ \text { F } & -1.44968 & -3.99520 & -0.83000 \\ \text { H } & -2.17516 & -3.40838 & -1.04231 \\ \text { F } & 0.49983 & -3.17218 & 0.46839 \\ \text { H } & -0.26292 & -3.49350 & -0.02038 \\ \text { F } & 2.85657 & -3.58089 & -0.26538 \\ \text { H } & 1.95433 & -3.44071 & 0.01834 \\ \text { F } & 4.56502 & -1.78781 & -0.55313 \\ \text { H } & 3.90685 & -2.46919 & -0.40887 \\ \text { F } & 3.97913 & 0.35442 & -1.64293\end{array}$




$\begin{array}{lccc}\text { H } & 4.22228 & -0.47988 & -1.22989 \\ & & & \\ \text { System (HF) } 20 & & \\ \text { F } & -3.11489 & 2.49272 & -2.36452 \\ \text { H } & -2.34813 & 2.79048 & -1.87768 \\ \text { F } & 3.11495 & -2.49270 & -2.36449 \\ \text { H } & 2.34817 & -2.79049 & -1.87770 \\ \text { F } & 1.17070 & 2.83108 & -2.06662 \\ \text { H } & 1.84045 & 2.36385 & -1.56840 \\ \text { F } & 1.08179 & -3.21271 & -1.08811 \\ \text { H } & 0.19782 & -3.10696 & -1.44735 \\ \text { F } & -2.86766 & -1.49727 & -0.79575 \\ \text { H } & -3.37969 & -0.81986 & -1.24879 \\ \text { F } & -4.15030 & 0.26475 & -1.96209 \\ \text { H } & -3.77236 & 1.13749 & -2.08893 \\ \text { F } & -1.08177 & 3.21263 & -1.08801 \\ \text { H } & -0.19779 & 3.10697 & -1.44725 \\ \text { F } & -1.17062 & -2.83093 & -2.06677 \\ \text { H } & -1.84040 & -2.36377 & -1.56853 \\ \text { F } & 4.15030 & -0.26471 & -1.96203 \\ \text { H } & 3.77238 & -1.13746 & -2.08887 \\ \text { F } & 2.86765 & 1.49727 & -0.79564 \\ \text { H } & 3.37968 & 0.81987 & -1.24869 \\ \text { F } & 1.17110 & -2.83102 & 2.06662 \\ \text { H } & 1.84082 & -2.36370 & 1.56843 \\ \text { F } & -1.08132 & -3.21267 & 1.08794 \\ \text { H } & -0.19735 & -3.10698 & 1.44719 \\ \text { F } & -3.11449 & -2.49307 & 2.36451 \\ \text { H } & -2.34771 & -2.79072 & 1.87765 \\ \text { F } & -4.15029 & -0.26528 & 1.96203 \\ \text { H } & -3.77221 & -1.13796 & 2.08888 \\ \text { F } & -2.86793 & 1.49694 & 0.79570 \\ \text { H } & -3.37986 & 0.81945 & 1.24873 \\ \text { F } & -1.17112 & 2.83091 & 2.06670 \\ \text { H } & -1.84083 & 2.36363 & 1.56847 \\ \text { F } & 1.08131 & 3.21274 & 1.08809 \\ \text { H } & 0.19733 & 3.10697 & 1.44732 \\ \text { F } & 3.11448 & 2.49305 & 2.36460 \\ \text { H } & 2.34769 & 2.79072 & 1.87776 \\ \text { F } & 4.15023 & 0.26523 & 1.96214 \\ \text { H } & 3.77216 & 1.13792 & 2.08899 \\ \text { F } & 2.86791 & -1.49695 & 0.79572 \\ \text { H } & 3.37981 & -0.81947 & 1.24878\end{array}$

(b) $\left(\mathrm{CO}_{2}\right)_{\mathrm{n}}(\mathrm{n}=1-20)$ systems, M06-2X/6-311+G(p,d) System (CO2) 1
C $\quad 0.00000$
$0.00000 \quad 0.00000$

O $\quad 0.00000$

0.00000

1.15466 


\begin{tabular}{lccc} 
O & 0.00000 & 0.00000 & -1.15466 \\
\multicolumn{4}{c}{ System (CO2)2 } \\
C & -1.14057 & -1.27509 & 0.00000 \\
O & -0.00008 & -1.46950 & 0.00000 \\
O & -2.27816 & -1.08890 & 0.00000 \\
C & 1.14057 & 1.27509 & 0.00000 \\
O & 2.27824 & 1.08937 & 0.00000 \\
O & -0.00000 & 1.46903 & 0.00000
\end{tabular}

System $(\mathrm{CO} 2) 3$

$\begin{array}{lccc}\text { C } & 0.00003 & 1.83911 & -0.00002 \\ \mathrm{O} & 1.06569 & 1.84024 & 0.44533 \\ \mathrm{O} & -1.06563 & 1.84039 & -0.44537 \\ \mathrm{C} & 1.76152 & -0.91726 & -0.35753 \\ \mathrm{O} & 2.58577 & -1.15876 & 0.40924 \\ \mathrm{O} & 0.93455 & -0.68334 & -1.13461 \\ \mathrm{C} & -1.76154 & -0.91722 & 0.35755 \\ \mathrm{O} & -0.93462 & -0.68319 & 1.13464 \\ \mathrm{O} & -2.58576 & -1.15882 & -0.40923\end{array}$

System (CO2)4

$\begin{array}{llll}C & -0.79720 & -0.71979 & 1.76634 \\ O & 0.33000 & -0.97520 & 1.76989 \\ O & -1.92337 & -0.46768 & 1.77085 \\ C & 0.88072 & 2.13999 & 0.22212 \\ O & 1.89690 & 2.67559 & 0.15302 \\ O & -0.15290 & 1.62142 & 0.29968 \\ C & -1.80961 & -0.03784 & -1.29530 \\ O & -1.04645 & -0.87961 & -1.06802 \\ O & -2.57201 & 0.79105 & -1.53372 \\ C & 1.72470 & -1.38337 & -0.69073 \\ O & 1.90931 & -0.24284 & -0.76263 \\ O & 1.55955 & -2.52198 & -0.63089\end{array}$

System (CO2) 5

$\begin{array}{lrrr}C & -0.59980 & -0.82110 & 1.81344 \\ O & -1.61648 & -0.55693 & 2.28838 \\ O & 0.42130 & -1.09997 & 1.34611 \\ C & 0.71159 & -1.38142 & -1.48158 \\ O & 0.57759 & -2.52409 & -1.47121 \\ O & 0.85569 & -0.23110 & -1.50769 \\ C & -0.04225 & 2.19371 & -0.39071 \\ O & -0.73225 & 1.54853 & 0.28116 \\ O & 0.62214 & 2.85047 & -1.06270 \\ C & -2.83132 & -0.20647 & -0.50368 \\ O & -1.99161 & -0.99114 & -0.65305 \\ O & -3.67305 & 0.56659 & -0.36680\end{array}$




$\begin{array}{llll}\mathrm{C} & 2.76316 & 0.21554 & 0.56933 \\ \mathrm{O} & 2.27845 & 1.21184 & 0.89896 \\ \mathrm{O} & 3.25718 & -0.77441 & 0.24175\end{array}$

System $(\mathrm{CO} 2) 6$

$\begin{array}{llll}\text { C } & -0.88511 & 2.02318 & -1.35984\end{array}$

$\begin{array}{llll}\mathrm{O} & -1.98743 & 2.33171 & -1.48453\end{array}$

$\begin{array}{llll}0 & 0.22959 & 1.72890 & -1.24722\end{array}$

$\begin{array}{llll}\text { C } & -2.20612 & 0.25121 & 1.35231\end{array}$

$\begin{array}{llll}0 & -1.39372 & 1.07004 & 1.24562\end{array}$

$\begin{array}{llll}0 & -3.02462 & -0.54996 & 1.47100\end{array}$

$\begin{array}{llll}\text { C } & 2.20607 & -0.25109 & -1.35226\end{array}$

$\begin{array}{llll}\text { O } & 3.02465 & 0.55004 & -1.47065\end{array}$

$\begin{array}{llll}\text { O } & 1.39359 & -1.06987 & -1.24587\end{array}$

$\begin{array}{llll}\text { C } & 0.88515 & -2.02328 & 1.35969\end{array}$

$\begin{array}{llll}\text { O } & -0.22959 & -1.72904 & 1.24732\end{array}$

$\begin{array}{llll}0 & 1.98751 & -2.33178 & 1.48412\end{array}$

$\begin{array}{llll}\text { C } & -1.31196 & -1.78648 & -1.35200\end{array}$

$\begin{array}{llll}\text { O } & -1.02869 & -2.89659 & -1.46735\end{array}$

$\begin{array}{llll}0 & -1.61382 & -0.67286 & -1.24914\end{array}$

$\begin{array}{llll}\text { C } & 1.31197 & 1.78646 & 1.35210\end{array}$

$\begin{array}{llll}\mathrm{O} & 1.02887 & 2.89663 & 1.46722\end{array}$

$\begin{array}{llll}0 & 1.61365 & 0.67277 & 1.24949\end{array}$

System $(\mathrm{CO} 2) 7$

$\begin{array}{llll}\text { C } & 0.25607 & -0.25862 & 1.86517\end{array}$

$\begin{array}{llll}0 & -0.16015 & -0.54843 & 2.89500\end{array}$

$\begin{array}{llll}0 & 0.68474 & 0.04448 & 0.82777\end{array}$

$\begin{array}{llll}\text { C } & 0.05161 & 0.11791 & -1.96516\end{array}$

$\begin{array}{llll}0 & -1.00505 & -0.27754 & -1.70628\end{array}$

$\begin{array}{llll}0 & 1.09867 & 0.51677 & -2.23447\end{array}$

$\begin{array}{llll}\text { C } & -2.86906 & 1.03276 & -0.05993\end{array}$

$\begin{array}{llll}\text { O } & -2.23388 & 0.67119 & 0.84225\end{array}$

$\begin{array}{llll}0 & -3.50826 & 1.40282 & -0.94064\end{array}$

$\begin{array}{llll}\text { C } & -2.12767 & -2.12554 & 0.08146\end{array}$

$\begin{array}{llll}0 & -1.09295 & -2.33381 & 0.55578\end{array}$

$\begin{array}{llll}0 & -3.16533 & -1.92532 & -0.37903\end{array}$

$\begin{array}{llll}\text { C } & 1.66218 & -2.45842 & -0.14356\end{array}$

$\begin{array}{llll}\mathrm{O} & 1.33322 & -2.27807 & -1.23684\end{array}$

$\begin{array}{llll}0 & 1.98465 & -2.64656 & 0.94847\end{array}$

$\begin{array}{llll}\text { C } & -0.01135 & 2.75209 & 0.50049\end{array}$

$\begin{array}{llll}\text { O } & -0.39085 & 2.55689 & -0.57549\end{array}$

$\begin{array}{llll}0 & 0.34043 & 2.94826 & 1.58060\end{array}$

$\begin{array}{llll}C & 3.04808 & 0.93497 & -0.29081\end{array}$

$\begin{array}{llll}\text { O } & 3.41877 & -0.15512 & -0.37895\end{array}$

$\begin{array}{llll}0 & 2.68860 & 2.02809 & -0.18892\end{array}$

System (CO2)8 


\begin{tabular}{|c|c|c|c|}
\hline C & 0.30659 & -0.18098 & -1.51600 \\
\hline $\mathrm{O}$ & 0.27871 & 0.21132 & -0.42151 \\
\hline $\mathrm{O}$ & 0.32594 & -0.56666 & -2.59678 \\
\hline C & -1.12607 & 0.21669 & 2.03247 \\
\hline $\mathrm{O}$ & -0.30913 & 0.85614 & 2.53477 \\
\hline $\mathrm{O}$ & -1.95360 & -0.42419 & 1.53865 \\
\hline C & 1.46558 & -1.95204 & 1.00396 \\
\hline $\mathrm{O}$ & 2.14235 & -2.06103 & 0.07352 \\
\hline $\mathrm{O}$ & 0.78914 & -1.85184 & 1.93377 \\
\hline C & 1.99077 & 1.65125 & 1.14566 \\
\hline $\mathrm{O}$ & 1.44120 & 2.61968 & 0.84483 \\
\hline $\mathrm{O}$ & 2.56006 & 0.68905 & 1.44347 \\
\hline C & -3.48779 & 0.34311 & -0.69937 \\
\hline $\mathrm{O}$ & -4.42536 & 0.57237 & -0.07492 \\
\hline $\mathrm{O}$ & -2.54963 & 0.12053 & -1.34597 \\
\hline C & -2.04496 & -2.54223 & -0.33130 \\
\hline 0 & -3.18962 & -2.58211 & -0.20088 \\
\hline $\mathrm{O}$ & -0.89702 & -2.51239 & -0.47406 \\
\hline C & -1.05852 & 2.67256 & -0.60100 \\
\hline $\mathrm{O}$ & -0.46606 & 2.90781 & -1.56123 \\
\hline $\mathrm{O}$ & -1.67616 & 2.43357 & 0.34862 \\
\hline C & 3.95909 & -0.21302 & -1.02092 \\
\hline $\mathrm{O}$ & 4.86336 & -0.78114 & -0.59008 \\
\hline $\mathrm{O}$ & 3.06231 & 0.37241 & -1.46234 \\
\hline
\end{tabular}

\begin{tabular}{|c|c|c|c|}
\hline \multicolumn{4}{|c|}{ System (CO2)9 } \\
\hline$C$ & 0.02599 & -0.38291 & -1.35939 \\
\hline & -0.01673 & -0.25323 & -0.20642 \\
\hline & 0.07782 & -0.52544 & -2.49858 \\
\hline & -1.62862 & 0.09920 & 1.95572 \\
\hline & -0.71799 & 0.32083 & 2.62890 \\
\hline & -2.55490 & -0.11707 & 1.29858 \\
\hline & 1.90927 & 0.14721 & 1.70731 \\
\hline & 1.94708 & -0.97724 & 1.96777 \\
\hline & 1.90031 & 1.27330 & 1.44867 \\
\hline & 0.08661 & -2.82326 & 0.86262 \\
\hline & 0.84097 & -3.06769 & 0.02143 \\
\hline & -0.66723 & -2.58983 & 1.70494 \\
\hline & -0.04246 & 3.19415 & 0.55826 \\
\hline & 0.81090 & 3.96479 & 0.63558 \\
\hline & -0.91478 & 2.43711 & 0.48055 \\
\hline & -3.16475 & -1.91682 & -0.84223 \\
\hline & -2.06048 & -2.25293 & -0.92996 \\
\hline & -4.26952 & -1.59832 & -0.76459 \\
\hline & 3.31751 & -1.83666 & -0.76380 \\
\hline & 2.77368 & -0.81976 & -0.89315 \\
\hline & 3.87203 & -2.83600 & -0.63903 \\
\hline C & -3.20474 & 1.47805 & -0.94106 \\
\hline
\end{tabular}




$\begin{array}{llll}\mathrm{O} & -3.87821 & 2.20705 & -0.35748 \\ \mathrm{O} & -2.53513 & 0.75046 & -1.54511 \\ \mathrm{C} & 2.69887 & 2.04024 & -1.17191 \\ \mathrm{O} & 1.57945 & 1.95379 & -1.45637 \\ \mathrm{O} & 3.81446 & 2.13078 & -0.89986\end{array}$

System (CO2)10

$\begin{array}{lccc}\mathrm{C} & -0.03529 & 0.14862 & -0.88766 \\ \mathrm{O} & 0.90998 & 0.51777 & -1.43358 \\ \mathrm{O} & -0.98124 & -0.21750 & -0.32959 \\ \mathrm{C} & -0.61967 & 1.14929 & 2.13372 \\ \mathrm{O} & -0.11438 & 1.95833 & 1.47473 \\ \mathrm{O} & -1.10936 & 0.34644 & 2.79746 \\ \mathrm{C} & 2.45800 & -0.75861 & 1.79738 \\ \mathrm{O} & 1.38207 & -0.64187 & 1.37642 \\ \mathrm{O} & 3.51592 & -0.87205 & 2.23142 \\ \mathrm{C} & 1.20908 & -3.01955 & -0.23605 \\ \mathrm{O} & 0.57915 & -2.54256 & -1.08278 \\ \mathrm{O} & 1.84774 & -3.49902 & 0.59426 \\ \mathrm{C} & -2.02231 & -2.08811 & 1.48390 \\ \mathrm{O} & -3.02990 & -1.52491 & 1.46617 \\ \mathrm{O} & -1.01585 & -2.65536 & 1.51340 \\ \mathrm{C} & 2.25417 & 2.57682 & -0.02067 \\ \mathrm{O} & 2.89139 & 1.74447 & 0.47112 \\ \mathrm{O} & 1.62671 & 3.41133 & -0.50820 \\ \mathrm{C} & -3.47236 & 0.79674 & -0.11030 \\ \mathrm{O} & -3.14248 & 1.48025 & 0.76349 \\ \mathrm{O} & -3.80754 & 0.13115 & -0.99028 \\ \mathrm{C} & 3.58182 & -0.25974 & -1.51606 \\ \mathrm{O} & 3.85940 & 0.61343 & -2.21406 \\ \mathrm{O} & 3.31501 & -1.14324 & -0.81645 \\ \mathrm{C} & -1.25245 & 3.54952 & -0.57500 \\ \mathrm{O} & -1.26310 & 2.59989 & -1.23919 \\ \mathrm{O} & -1.25888 & 4.49836 & 0.07678 \\ \mathrm{C} & -2.10305 & -2.09750 & -2.07244 \\ \mathrm{O} & -1.69287 & -1.44000 & -2.92836 \\ \mathrm{O} & -2.51024 & -2.76303 & -1.22036\end{array}$

System (CO2)11

$\begin{array}{lccc}\text { C } & -0.05455 & -0.05705 & -0.85086 \\ \text { O } & 0.50887 & -0.51716 & 0.04933 \\ \text { O } & -0.60878 & 0.40368 & -1.75084 \\ \text { C } & -0.02262 & -1.72550 & 2.48703 \\ \text { O } & -1.10158 & -1.96773 & 2.15032 \\ \text { O } & 1.05040 & -1.48941 & 2.84111 \\ \text { C } & 2.94165 & -0.19671 & 1.24401 \\ \text { O } & 3.30333 & -1.21886 & 0.84665 \\ \text { O } & 2.60540 & 0.83962 & 1.62974\end{array}$




\begin{tabular}{|c|c|c|c|}
\hline 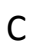 & -2.94146 & 0.20252 & 1.37427 \\
\hline ? & -3.34821 & 0.59206 & 2.37689 \\
\hline b & -2.55350 & -0.19504 & 0.35597 \\
\hline E & 0.07984 & 2.08272 & 2.33492 \\
\hline b & -0.42858 & 1.04472 & 2.27603 \\
\hline o & 0.58041 & 3.11819 & 2.40977 \\
\hline C & -2.26404 & -2.82468 & -0.47546 \\
\hline $\mathrm{O}$ & -1.23744 & -2.58779 & -0.95683 \\
\hline 0 & -3.28320 & -3.07899 & -0.00321 \\
\hline C & 1.50999 & -3.06670 & -0.43547 \\
\hline 0 & 1.86322 & -2.66622 & -1.46053 \\
\hline 0 & 1.16237 & -3.47804 & 0.58546 \\
\hline C & 2.19763 & 2.75907 & -0.43118 \\
\hline 0 & 1.08529 & 2.43453 & -0.41379 \\
\hline 0 & 3.30042 & 3.08756 & -0.45350 \\
\hline C & -1.56969 & 3.08162 & -0.87309 \\
\hline b & -1.77576 & 2.61020 & 0.16571 \\
\hline $\mathrm{O}$ & -1.36278 & 3.56853 & -1.89536 \\
\hline C & 3.51157 & -0.44703 & -2.12548 \\
\hline $\mathrm{O}$ & 4.29399 & -1.15512 & -2.5814 \\
\hline $\mathrm{O}$ & 2.73385 & 0.28392 & -1.66946 \\
\hline C & -3.38568 & 0.18708 & -2.24752 \\
\hline $\mathrm{O}$ & -3.31681 & -0.95312 & -2.41528 \\
\hline 0 & -3.47288 & 1.32795 & -2.0876 \\
\hline
\end{tabular}

\begin{tabular}{|c|c|c|c|}
\hline \multicolumn{4}{|c|}{ System (CO2)12 } \\
\hline$C$ & 0.02663 & 0.07401 & -0.47116 \\
\hline O & -0.33172 & -0.98447 & -0.18359 \\
\hline D & 0.39749 & 1.12736 & -0.76888 \\
\hline C & -2.73594 & 1.29824 & 1.50044 \\
\hline D & -3.27063 & 1.24953 & 2.51704 \\
\hline D & -2.20889 & 1.36426 & 0.46881 \\
\hline C & 2.99253 & -0.38005 & 1.49596 \\
\hline b & 1.95659 & 0.14222 & 1.48917 \\
\hline b & 4.02239 & -0.89073 & 1.51477 \\
\hline C & -2.51179 & -2.28222 & 0.76514 \\
\hline b & -3.18271 & -1.40244 & 0.42812 \\
\hline b & -1.85604 & -3.16612 & 1.11151 \\
\hline C & 0.97233 & -3.24995 & 0.63034 \\
\hline 0 & 0.71325 & -3.79970 & -0.35021 \\
\hline $\mathrm{O}$ & 1.24743 & -2.70874 & 1.61506 \\
\hline C & 0.90925 & 2.62898 & 1.74441 \\
\hline $\mathrm{O}$ & -0.01917 & 2.16335 & 2.25393 \\
\hline 0 & 1.84464 & 3.09146 & 1.25282 \\
\hline C & -0.10129 & -0.66466 & 3.29191 \\
\hline 0 & -0.97394 & -0.64978 & 2.53133 \\
\hline $\mathrm{O}$ & 0.75479 & -0.67856 & 4.06297 \\
\hline C & -0.79142 & -2.53153 & -2.41677 \\
\hline
\end{tabular}




$\begin{array}{lccc}\text { O } & -1.75092 & -2.98776 & -1.96648 \\ \text { O } & 0.16603 & -2.07600 & -2.87852 \\ \text { C } & -3.13797 & 0.44058 & -1.98674 \\ \text { O } & -2.10214 & -0.01518 & -2.23425 \\ \text { O } & -4.17384 & 0.88772 & -1.75635 \\ \text { C } & 2.81185 & -1.16555 & -2.06879 \\ \text { O } & 3.18578 & -0.93339 & -3.13120 \\ \text { O } & 2.43780 & -1.39539 & -0.99513 \\ \text { C } & 2.73501 & 2.40533 & -1.35979 \\ \text { O } & 3.31643 & 1.47997 & -0.97545 \\ \text { O } & 2.16471 & 3.32235 & -1.76090 \\ \text { C } & -1.16494 & 3.42430 & -1.12318 \\ \text { O } & -1.69044 & 2.96721 & -2.04391 \\ \text { O } & -0.65008 & 3.89474 & -0.20203\end{array}$

\begin{tabular}{|c|c|c|c|}
\hline \multicolumn{4}{|c|}{ System (CO2) 13} \\
\hline ( & -0.00008 & -0.00049 & 0.00030 \\
\hline $\mathrm{O}$ & 0.02086 & -0.02008 & -1.15356 \\
\hline 0 & -0.02091 & 0.01979 & 1.15428 \\
\hline 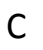 & 0.82021 & -1.85630 & 2.93337 \\
\hline $\mathrm{O}$ & 1.78431 & -2.02596 & 2.31693 \\
\hline $\mathrm{O}$ & -0.14008 & -1.70514 & 3.55476 \\
\hline C & 1.15647 & $1.7 €$ & 2.87358 \\
\hline $\mathrm{O}$ & 1.50000 & 0.88428 & 3.53790 \\
\hline 0 & 0.82870 & 2.65 & 2.21350 \\
\hline C & -2.14475 & 0.2 & 2.83876 \\
\hline O & -2.74094 & -0.51179 & 2.20231 \\
\hline $\mathrm{O}$ & -1.56466 & 1.0 & 3.48026 \\
\hline C & -0.82141 & 1.8 & -2.93238 \\
\hline O & -1.78507 & 2.02595 & -2.31582 \\
\hline $\mathrm{O}$ & 0.13801 & 1.7 & -3 \\
\hline C & 2.1 & -0.2 & $-2 . \varepsilon$ \\
\hline O & 2.74183 & 0.51180 & -2.20310 \\
\hline 0 & 1.56654 & -1.0 & -3.47998 \\
\hline C & -1.15506 & -1.7 & -2.87423 \\
\hline $\mathrm{O}$ & -0.82577 & -2.65392 & -2.21487 \\
\hline $\mathrm{O}$ & -1.50023 & -0.88345 & -3.53723 \\
\hline C & -1.73053 & 3.14121 & 0.38103 \\
\hline $\mathrm{O}$ & -1.56544 & 4.25643 & 0.15177 \\
\hline $\mathrm{O}$ & -1.91223 & 2.02133 & 0.61994 \\
\hline C & 1.73062 & -3.14158 & -0.38312 \\
\hline $\mathrm{O}$ & 1.56161 & -4.25673 & -0.15603 \\
\hline $\mathrm{O}$ & 1.91270 & -2.02127 & -0.61923 \\
\hline C & 3.57049 & -0.06490 & 0.53527 \\
\hline $\mathrm{O}$ & 4.46090 & -0.76849 & 0.34584 \\
\hline $\mathrm{O}$ & 2.68210 & 0.65238 & 0.73608 \\
\hline C & -1.86379 & -3.05155 & 0.48338 \\
\hline $\mathrm{O}$ & -2.91027 & -3.47240 & 0.25622 \\
\hline
\end{tabular}




$\begin{array}{llll}\mathrm{O} & -0.80751 & -2.63792 & 0.72377 \\ \mathrm{C} & 1.86498 & 3.05100 & -0.48530 \\ \mathrm{O} & 0.80822 & 2.63799 & -0.72391 \\ \mathrm{O} & 2.91075 & 3.47343 & -0.25812 \\ \mathrm{C} & -3.57091 & 0.06564 & -0.53282 \\ \mathrm{O} & -4.46143 & 0.76873 & -0.34147 \\ \mathrm{O} & -2.68311 & -0.65225 & -0.73422\end{array}$

\begin{tabular}{|c|c|c|c|}
\hline \multicolumn{4}{|c|}{ System (CO2)14 } \\
\hline r & -0.38072 & -0.00406 & 0.00702 \\
\hline $\mathrm{O}$ & -1.18251 & 0.47114 & 0.68827 \\
\hline b & 0.41972 & -0.48058 & -0.67493 \\
\hline & 2.19473 & 0.70417 & -2.34258 \\
\hline U & 3.02397 & -0.00611 & -1.96438 \\
\hline 0 & 1.37891 & 1.42354 & -2.73086 \\
\hline C & 2.52020 & -2.09205 & -0.03864 \\
\hline $\mathrm{O}$ & 2.25362 & -2.80995 & -0.89999 \\
\hline $\mathrm{O}$ & 2.79508 & -1.38060 & 0.83308 \\
\hline C & 2.42257 & 1.91 & 1.30330 \\
\hline $\mathrm{O}$ & 2.94135 & 2.11708 & 2.30996 \\
\hline $\mathrm{O}$ & 1.89465 & 1.72041 & 0.28894 \\
\hline C & -0.89697 & 2.2 & 2.70345 \\
\hline 0 & -0.17125 & 2.89347 & 2.05255 \\
\hline D & -1.61352 & 1.65709 & 3.36745 \\
\hline C & -3.32894 & 2.01769 & 0.03049 \\
\hline $\mathrm{O}$ & -3.08470 & 2.74037 & 0.89610 \\
\hline $\mathrm{O}$ & -3.58264 & 1.30757 & -0.84659 \\
\hline C & -2.93889 & -0.70785 & 2.39754 \\
\hline $\mathrm{O}$ & -3.78588 & -0.0 & 2.01139 \\
\hline D & -2.10260 & -1.4 & 2.78936 \\
\hline C & 1.03448 & -1.0 & 3.15685 \\
\hline 0 & 0.82627 & -0.0 & 2.58210 \\
\hline 0 & 1.24677 & -1.9 & 3.73963 \\
\hline C & -3.24480 & -1.8 & -1.25518 \\
\hline 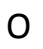 & -2.65096 & -1.72205 & -0.26249 \\
\hline $\mathrm{O}$ & -3.84515 & -1.88670 & -2.23293 \\
\hline C & -1.82842 & 1.02930 & -3.14655 \\
\hline $\mathrm{O}$ & -2.08142 & 1.99498 & -3.71863 \\
\hline $\mathrm{O}$ & -1.57651 & 0.04777 & -2.58323 \\
\hline C & 0.09822 & -2.23997 & -2.73074 \\
\hline $\mathrm{O}$ & 0.84601 & -1.63113 & -3.36480 \\
\hline $\mathrm{O}$ & -0.65837 & -2.85628 & -2.11031 \\
\hline C & 0.06435 & 3.42068 & -1.02681 \\
\hline $\mathrm{O}$ & -0.75670 & 2.60414 & -1.08771 \\
\hline 0 & 0.86792 & 4.24230 & -0.97364 \\
\hline C & -0.86989 & -3.41456 & 1.09976 \\
\hline $\mathrm{O}$ & -1.69171 & -4.21966 & 1.10806 \\
\hline 0 & -0.03109 & -2.61407 & 1.09663 \\
\hline
\end{tabular}




\begin{tabular}{|c|c|c|c|}
\hline & 5.15420 & -0.08711 & -0.15908 \\
\hline b & 5.45965 & -1.13300 & -0.53919 \\
\hline & 4.86099 & 0.96207 & 0.22707 \\
\hline \multicolumn{4}{|c|}{ System (CO2)15 } \\
\hline & -0.69268 & -0.02705 & 0.01611 \\
\hline & -0.04470 & 0.58898 & -0.71447 \\
\hline & -1.33969 & -0.64488 & 0.74542 \\
\hline & 2.17204 & -0.15835 & -2.09152 \\
\hline & 1.67471 & -1.17235 & -2.33412 \\
\hline b & 2.66942 & 0.85706 & -1.85373 \\
\hline & 2.43646 & -0.31447 & 1.80603 \\
\hline b & 2.05361 & -0.64132 & 0.75871 \\
\hline 0 & 2.81745 & 0.00722 & 2.84120 \\
\hline & 1.29500 & 2.92646 & -0.40078 \\
\hline o & 0.87147 & 3.32567 & -1.39545 \\
\hline b & 1.72329 & 2.53867 & 0.60340 \\
\hline & 1.16703 & -3.10170 & -0.28285 \\
\hline b & 2.24858 & -3.46056 & -0.11423 \\
\hline 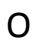 & 0.07576 & -2.75435 & -0.45490 \\
\hline & -1.26664 & -2.09123 & -2.86169 \\
\hline ) & -1.49904 & -1.01035 & -2.51194 \\
\hline כ & -1.04061 & -3.16013 & -3.22199 \\
\hline & -2.49231 & 3.11122 & 0.23848 \\
\hline ) & -3.53807 & 3.52326 & -0.00721 \\
\hline $\mathrm{O}$ & -1.43348 & 2.71115 & 0.48987 \\
\hline & -0.62686 & -1.71285 & 3.14591 \\
\hline 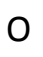 & 0.35567 & -2.10531 & 2.67896 \\
\hline 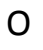 & -1.59900 & -1.31956 & 3.62746 \\
\hline E & -0.77986 & 1.68815 & -3.10694 \\
\hline D & 0.18891 & 1.29361 & -3.59364 \\
\hline כ & -1.75906 & 2.08371 & -2.63506 \\
\hline C & -3.58546 & 0.10289 & 2.08914 \\
\hline 0 & -3.11851 & 1.13384 & 2.32837 \\
\hline כ & -4.06792 & -0.91792 & 1.85181 \\
\hline C & -0.18579 & 2.06629 & 2.89568 \\
\hline . & -0.41633 & 3.12476 & 3.28372 \\
\hline . & 0.05444 & 0.99722 & 2.51741 \\
\hline C & -2.64905 & -2.99887 & 0.41707 \\
\hline $\mathrm{O}$ & -2.26214 & -3.36642 & 1.44002 \\
\hline $\mathrm{O}$ & -3.03819 & -2.64769 & -0.61399 \\
\hline C & -3.85505 & 0.28995 & -1.71193 \\
\hline $\mathrm{O}$ & -4.32708 & -0.02158 & -2.71396 \\
\hline $\mathrm{O}$ & -3.39321 & 0.60916 & -0.69759 \\
\hline C & 4.48012 & 2.00089 & -0.10039 \\
\hline $\mathrm{O}$ & 4.41983 & 3.00015 & -0.67160 \\
\hline $\mathrm{O}$ & 4.55435 & 1.00430 & 0.48340 \\
\hline 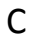 & 4.58232 & -1.78749 & -0.05550 \\
\hline
\end{tabular}




\begin{tabular}{|c|c|c|c|}
\hline & 4.73596 & -2.01051 & 1.06610 \\
\hline & 4.43416 & -1.56121 & -1.17958 \\
\hline \multicolumn{4}{|c|}{ System (CO2)16 } \\
\hline${ }^{\circ}$ & 0.92331 & -0.02968 & 0.00475 \\
\hline & 0.28849 & -0.14276 & 0.96274 \\
\hline & 1.55659 & 0.07937 & -0.95390 \\
\hline & -2.36149 & -0.04461 & -1.44219 \\
\hline 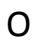 & -1.72665 & -0.84971 & -0.89866 \\
\hline 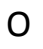 & -2.99367 & 0.75129 & -1.98100 \\
\hline 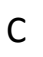 & -1.45156 & -2.11277 & 1.68453 \\
\hline & -2.18896 & -1.32453 & $2.0967 \varepsilon$ \\
\hline $\mathrm{O}$ & -0.71284 & -2.90138 & 1.27624 \\
\hline E & -1.57729 & 1.46960 & 2.05199 \\
\hline 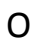 & -2.08536 & 1.57763 & 1.01534 \\
\hline $\mathrm{O}$ & -1.07888 & 1.37614 & 3.08570 \\
\hline 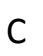 & -0.18842 & -3.10061 & -1.53751 \\
\hline כ & 0.81074 & -2.61696 & -1.20705 \\
\hline $\mathrm{O}$ & -1.17504 & -3.59131 & -1.8725 \\
\hline 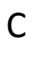 & -0.22244 & 3.17998 & -1.24709 \\
\hline כ & -0.03508 & 4.30203 & -1.07781 \\
\hline O & -0.40987 & 2.04877 & -1.42483 \\
\hline C & 1.94683 & 3.04700 & 1.60736 \\
\hline b & 0.96903 & 2.58085 & 1.19309 \\
\hline $\mathrm{O}$ & 2.90829 & 3.52053 & 2.02552 \\
\hline C & 2.38912 & -3.10060 & 1.11214 \\
\hline $\mathrm{O}$ & 2.34437 & -1.99615 & 1.46311 \\
\hline $\mathrm{O}$ & 2.44011 & -4.19989 & 0.77712 \\
\hline E & 0.65723 & 0.42815 & -3.50516 \\
\hline b & -0.14931 & -0.38379 & -3.34038 \\
\hline 0 & 1.45165 & 1.24705 & -3.68035 \\
\hline . & 3.37055 & 1.96717 & -1.64534 \\
\hline b & 2.67030 & 2.78874 & -1.22932 \\
\hline 0 & 4.08183 & 1.15813 & -2.05788 \\
\hline 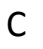 & 1.16475 & -0.40804 & 3.53663 \\
\hline $\mathrm{O}$ & 0.40 & -1.2 & 3.71238 \\
\hline $\mathrm{O}$ & 1.94058 & 0.43414 & 3.37315 \\
\hline C & 3.31786 & -1.63540 & -2.12301 \\
\hline $\mathrm{O}$ & 3.80392 & -1.85474 & -1.09686 \\
\hline $\mathrm{O}$ & 2.83776 & -1.43248 & -3.1525 \\
\hline C & 4.19171 & 0.12031 & 1.58765 \\
\hline $\mathrm{O}$ & 4.86982 & -0.53825 & 2.24371 \\
\hline $\mathrm{O}$ & 3.51949 & 0.79355 & 0.92510 \\
\hline C & -4.51613 & 0.04818 & 1.38153 \\
\hline $\mathrm{O}$ & -4.46848 & -0.49056 & 0.3582 \\
\hline $\mathrm{O}$ & -4.56858 & 0.58050 & 2.4024 \\
\hline C & -3.77722 & -2.89358 & -0.8907 \\
\hline 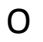 & -3.47894 & -3.33133 & 0.1368 \\
\hline
\end{tabular}




$\begin{array}{llll}\mathrm{O} & -4.07852 & -2.45775 & -1.91581 \\ \mathrm{C} & -3.86776 & 3.05784 & -0.58096 \\ \mathrm{O} & -3.00522 & 3.65347 & -1.06585 \\ \mathrm{O} & -4.73700 & 2.47866 & -0.08857\end{array}$

System (CO2)17

\begin{tabular}{|c|c|c|c|}
\hline & -1.13247 & 0.02052 & -0.00164 \\
\hline & -0.77349 & -0.67941 & 0.84443 \\
\hline & -1.48894 & 0.71586 & -0.85051 \\
\hline & 2.30008 & 0.19830 & -0.94583 \\
\hline & 3.17886 & 0.54657 & -0.93885 \\
\hline & 1.42173 & -0.95406 & -0.95424 \\
\hline & 0.35568 & -3.16870 & 0.53185 \\
\hline & 1.11365 & -2.94494 & 1.37461 \\
\hline ) & -0.40341 & -3.39411 & -0.30936 \\
\hline & 1.08082 & -0.47213 & 2.80138 \\
\hline & 1.85801 & -0.03426 & 2.06124 \\
\hline ) & 0.31972 & -0.90386 & 3.55025 \\
\hline & 0.84909 & 2.93150 & 0.71227 \\
\hline & 0.89502 & 3.93914 & 1.26396 \\
\hline ) & 0.80521 & 1.91303 & 0.15782 \\
\hline & 0.12781 & 2.16587 & -2.56512 \\
\hline & -0.47362 & 3.11527 & -2.30315 \\
\hline b & 0.73563 & 1.21869 & -2.83190 \\
\hline- & -0.29524 & -2.12839 & -2.85981 \\
\hline ) & -1.23042 & -1.57633 & -2.45885 \\
\hline ) & 0.63044 & -2.67848 & -3.26972 \\
\hline & -3.37038 & -2.60485 & -0.89607 \\
\hline D & -3.19483 & -1.87034 & -0.01566 \\
\hline ) & -3.55404 & -3.34310 & -1.75874 \\
\hline 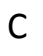 & -2.25310 & -1.95193 & 2.73261 \\
\hline כ & -1.72938 & -2.94983 & 2.48552 \\
\hline 0 & -2.79199 & -0.96055 & 2.98656 \\
\hline 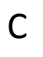 & -2.70424 & 3.14149 & -0.52412 \\
\hline כ & -3.42037 & 2.90624 & -1.39734 \\
\hline b & -1.99742 & 3.38795 & 0.35799 \\
\hline : & -1.91478 & 2.04200 & 2.96732 \\
\hline $\mathrm{O}$ & -2.88867 & 2.43099 & 3.44186 \\
\hline $\mathrm{O}$ & -0.92536 & 1.65511 & 2.50353 \\
\hline C & -4.57207 & 0.28369 & 1.12737 \\
\hline $\mathrm{O}$ & -3.65338 & 0.96889 & 0.95335 \\
\hline $\mathrm{O}$ & -5.49169 & -0.38588 & 1.3008 \\
\hline C & -3.14025 & 0.47128 & -2.95555 \\
\hline 0 & -2.37820 & 1.03949 & -3.61010 \\
\hline $\mathrm{O}$ & -3.90982 & -0.10647 & -2.3143 \\
\hline C & 3.79176 & -2.11136 & 1.68976 \\
\hline $\mathrm{O}$ & 3.76267 & -2.23786 & 2.83452 \\
\hline ? & 3.82647 & -1.99042 & 0.5388 \\
\hline
\end{tabular}




\begin{tabular}{|c|c|c|c|}
\hline & 4.17652 & 1.53045 & 1.50771 \\
\hline J & 3.56825 & 2.48406 & 1.26478 \\
\hline & 4.78856 & 0.58703 & 1.76277 \\
\hline & 3.01705 & -3.22799 & -1.82276 \\
\hline & 2.43719 & -4.01440 & -1.20487 \\
\hline & 3.59874 & -2.44376 & -2.43786 \\
\hline & 3.68363 & 3.27387 & -1.50639 \\
\hline & 2.54469 & 3.46850 & -1.57656 \\
\hline & 4.82027 & 3.09348 & -1.44556 \\
\hline \multicolumn{4}{|c|}{ System (CO2)18 } \\
\hline 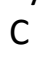 & -0.95314 & -0.49576 & 0.12460 \\
\hline & -0.31492 & -0.14407 & -0.77129 \\
\hline $\mathrm{O}$ & -1.58843 & -0.85045 & 1.02033 \\
\hline & 1.73864 & 1.59637 & -0.90143 \\
\hline & 2.21720 & 1.06348 & 0.00765 \\
\hline b & 1.26743 & 2.12188 & -1.81328 \\
\hline & 2.27167 & -1.52022 & 1.27270 \\
\hline ) & 1.58983 & -1.90006 & 0.41353 \\
\hline 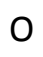 & 2.94950 & -1.14530 & 2.12306 \\
\hline & 1.26697 & -1.60924 & -2.45730 \\
\hline J & 2.08100 & -0.79011 & -2.42412 \\
\hline b & 0.45062 & -2.42578 & -2.49493 \\
\hline & -1.80588 & 3.02063 & 0.27101 \\
\hline 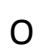 & -2.75830 & 3.64974 & 0.13838 \\
\hline D & -0.83973 & 2.38935 & 0.40561 \\
\hline 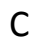 & 0.42902 & 1.57521 & 2.72941 \\
\hline 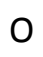 & 0.49250 & 0.47643 & 2.36651 \\
\hline b & 0.36150 & 2.66547 & 3.09394 \\
\hline & -1.11526 & 1.07328 & -3.07180 \\
\hline b & -1.78133 & 1.79522 & -2.45723 \\
\hline ) & -0.45950 & 0.35787 & -3.69307 \\
\hline & -0.15081 & -4.01106 & -0.18240 \\
\hline b & 0.77734 & -4.69281 & -0.17526 \\
\hline כ & -1.09225 & -3.33632 & -0.18898 \\
\hline E & -2.61950 & -2.41928 & -2.40777 \\
\hline 0 & -2.48717 & -1.29943 & -2.13633 \\
\hline 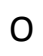 & -2.75766 & -3.52603 & -2.68901 \\
\hline C & -3.26641 & 0.57046 & 2.61373 \\
\hline 0 & -2.49231 & 1.42906 & 2.65439 \\
\hline 0 & -4.05096 & -0.27459 & 2.57472 \\
\hline C & -0.73573 & -1.90930 & 3.37378 \\
\hline 0 & -1.47261 & -1.23835 & 3.95619 \\
\hline 0 & 0.01267 & -2.58071 & 2.80293 \\
\hline C & -3.52615 & -2.73779 & 1.16200 \\
\hline 0 & -3.06089 & -3.14672 & 2.13525 \\
\hline O & -3.99858 & -2.34317 & 0.18248 \\
\hline - & -4.24117 & 0.54510 & -1.0323 \\
\hline
\end{tabular}




$\begin{array}{llll}\mathrm{O} & -5.01188 & 0.29540 & -1.84958 \\ \mathrm{O} & -3.47287 & 0.80839 & -0.20533 \\ \mathrm{C} & 4.51851 & -0.20555 & -1.15041 \\ \mathrm{O} & 4.64538 & 0.75701 & -1.77117 \\ \mathrm{O} & 4.39518 & -1.17629 & -0.53182 \\ \mathrm{C} & 0.94055 & 4.54681 & 1.00332 \\ \mathrm{O} & 0.05010 & 5.18547 & 1.35988 \\ \mathrm{O} & 1.83719 & 3.90869 & 0.64332 \\ \mathrm{C} & 4.01783 & 1.51987 & 2.04299 \\ \mathrm{O} & 3.19080 & 1.83576 & 2.78494 \\ \mathrm{O} & 4.85479 & 1.21643 & 1.30768 \\ \mathrm{C} & 3.45238 & -3.80446 & -0.70632 \\ \mathrm{O} & 3.13569 & -3.60556 & -1.80047 \\ \mathrm{O} & 3.77197 & -4.00418 & 0.38410 \\ \mathrm{C} & -0.21855 & 4.25819 & -2.67859 \\ \mathrm{O} & -0.11275 & 3.93681 & -3.78051 \\ \mathrm{O} & -0.33078 & 4.59253 & -1.57640\end{array}$

System (CO2)19

\begin{tabular}{|c|c|c|c|}
\hline E & -0.09857 & 1.00770 & 0.23842 \\
\hline & -0.00093 & 0.30592 & -0.67355 \\
\hline & -0.19241 & 1.70823 & 1.15059 \\
\hline & 0.44736 & -2.35222 & -0.80254 \\
\hline & -0.40021 & -2.48129 & -1.57368 \\
\hline & 1.30404 & -2.22031 & -0.03552 \\
\hline & -2.89232 & -1.14161 & 0.94120 \\
\hline & -1.74008 & -1.27702 & 0.92097 \\
\hline & -4.03528 & -1.01208 & 0.96141 \\
\hline & 3.19998 & -0.23733 & 0.84318 \\
\hline & 3.64205 & -0.93324 & 1.64521 \\
\hline & 2.75520 & 0.46454 & 0.03256 \\
\hline & 1.81774 & 0.37676 & -2.71457 \\
\hline & 1.70382 & 1.52537 & -2.76423 \\
\hline & 1.92619 & -0.77248 & -2.66894 \\
\hline & -1.77899 & -0.20102 & -2.65798 \\
\hline & -2.62221 & -0.32498 & -1.87186 \\
\hline & -0.95130 & -0.07552 & -3.44844 \\
\hline & 0.14664 & -1.38928 & 2.93159 \\
\hline & 0.81327 & -0.59550 & 2.41322 \\
\hline & -0.51829 & -2.17322 & 3.45052 \\
\hline & 2.66029 & 3.18230 & -0.63607 \\
\hline & 3.79122 & 3.10439 & -0.83909 \\
\hline & 1.52381 & 3.27379 & -0.43204 \\
\hline & -3.39214 & 2.49232 & -0.25050 \\
\hline & -2.90046 & 1.64376 & 0.36978 \\
\hline & -3.88261 & 3.33588 & -0.85910 \\
\hline & -0.61644 & 3.48570 & -2.31570 \\
\hline & -1.16944 & 2.54541 & -1.9213 \\
\hline
\end{tabular}




$\begin{array}{llll}\mathrm{O} & -0.07699 & 4.41865 & -2.71779 \\ \mathrm{C} & -2.07256 & 1.74231 & 3.10300 \\ \mathrm{O} & -2.01911 & 0.58894 & 3.17109 \\ \mathrm{O} & -2.14161 & 2.89239 & 3.03908 \\ \mathrm{C} & 1.54176 & 2.05370 & 3.21454 \\ \mathrm{O} & 0.66572 & 2.02078 & 3.96552 \\ \mathrm{O} & 2.42846 & 2.07989 & 2.47305 \\ \mathrm{C} & -0.44181 & 4.40494 & 1.27166 \\ \mathrm{O} & -1.22280 & 4.36229 & 0.41909 \\ \mathrm{O} & 0.34066 & 4.46160 & 2.11731 \\ \mathrm{C} & 3.62784 & -2.75652 & -1.63313 \\ \mathrm{O} & 3.00191 & -3.58759 & -2.12857 \\ \mathrm{O} & 4.26058 & -1.92123 & -1.14104 \\ \mathrm{C} & -3.05009 & -3.20988 & -1.92366 \\ \mathrm{O} & -3.10051 & -3.35512 & -0.77634 \\ \mathrm{O} & -2.99632 & -3.07251 & -3.06667 \\ \mathrm{C} & -1.63918 & -4.05902 & 1.58813 \\ \mathrm{O} & -0.62691 & -4.15097 & 1.03344 \\ \mathrm{O} & -2.64690 & -3.97033 & 2.13997 \\ \mathrm{C} & 2.76446 & -3.67021 & 1.80600 \\ \mathrm{O} & 3.44462 & -4.00002 & 0.93317 \\ \mathrm{O} & 2.08543 & -3.35567 & 2.68588 \\ \mathrm{C} & 5.16374 & 0.69263 & -1.55826 \\ \mathrm{O} & 5.72343 & 0.67100 & -0.54963 \\ \mathrm{O} & 4.60584 & 0.71299 & -2.57086 \\ \mathrm{C} & -5.38280 & -0.42060 & -1.43729 \\ \mathrm{O} & -5.36256 & -1.53302 & -1.74656 \\ \mathrm{O} & -5.41296 & 0.69577 & -1.14261\end{array}$

System (CO2)20

$\begin{array}{lrrr}C & 1.68152 & -0.04997 & -0.08903 \\ O & 1.64741 & -1.08422 & 0.42414 \\ O & 1.71229 & 0.98436 & -0.60035 \\ C & -1.84271 & -0.02638 & 0.41199 \\ O & -2.77903 & 0.01806 & -0.25979 \\ O & -0.91018 & -0.06721 & 1.09551 \\ C & -0.41931 & 2.36963 & -1.75296 \\ O & -0.98227 & 2.29101 & -0.74269 \\ O & 0.14147 & 2.45396 & -2.75490 \\ C & 0.67964 & -1.66335 & 2.91601 \\ O & 0.18449 & -2.61640 & 2.48957 \\ O & 1.17297 & -0.71079 & 3.34381 \\ C & 0.52582 & -3.50386 & -0.19925 \\ O & -0.47601 & -3.00668 & -0.50261 \\ O & 1.51645 & -4.01334 & 0.09432 \\ C & -0.32516 & -0.90069 & -2.94030 \\ O & -0.31753 & -0.41766 & -1.88538 \\ O & -0.33053 & -1.38169 & -3.98463\end{array}$




\begin{tabular}{lccc} 
C & 0.22346 & 1.85675 & 2.72206 \\
O & 1.15774 & 1.91913 & 2.03967 \\
O & -0.70237 & 1.78492 & 3.40331 \\
C & 2.53221 & 3.45864 & 0.08103 \\
O & 1.55378 & 3.89694 & -0.35192 \\
O & 3.51428 & 3.03814 & 0.51860 \\
C & 3.76367 & 0.98694 & 2.61009 \\
O & 3.75989 & 1.68784 & 3.52168 \\
O & 3.78307 & 0.26983 & 1.69809 \\
C & -3.63536 & 0.60094 & -2.86671 \\
O & -2.55938 & 0.92662 & -3.14065 \\
O & -4.71081 & 0.27446 & -2.60838 \\
C & -2.32154 & -3.51022 & 1.61625 \\
O & -1.95101 & -4.59797 & 1.67543 \\
O & -2.70206 & -2.41604 & 1.56179 \\
C & 3.74121 & -2.61687 & 1.25992 \\
O & 3.24716 & -2.73941 & 2.29529 \\
O & 4.25259 & -2.49710 & 0.22950 \\
C & 2.77384 & 1.51014 & -3.05764 \\
O & 3.18976 & 2.50170 & -2.63900 \\
O & 2.36570 & 0.51672 & -3.48730 \\
C & 3.13511 & -2.17630 & -2.71380 \\
O & 2.10026 & -2.11935 & -2.19426 \\
O & 4.15802 & -2.24852 & -3.23622 \\
C & -3.61555 & 2.77791 & -0.04689 \\
O & -3.97757 & 2.77073 & -1.14322 \\
O & -3.24204 & 2.79328 & 1.04691 \\
C & 5.27855 & -0.01361 & -0.65897 \\
O & 6.25879 & -0.02348 & -0.05640 \\
O & 4.29773 & 0.00161 & -1.27665 \\
C & -3.12544 & -2.96044 & -1.64338 \\
O & -3.39216 & -3.86596 & -0.98091 \\
O & -2.84252 & -2.06677 & -2.32126 \\
C & -2.60348 & -0.37256 & 3.46463 \\
O & -3.38263 & 0.19786 & 2.83119 \\
O & -1.82707 & -0.95284 & 4.09383 \\
O & -5.40963 & -0.46502 & 0.05701 \\
\hline & -5.25081 & -1.57318 & -0.23095 \\
\hline & -1.5641789 & 0.63783 & 0.35423 \\
\hline
\end{tabular}

(c) $\mathrm{Cl}_{2} \mathrm{Ar}_{n}$ ( $\mathrm{n}=1-20$ ) systems, MP2/aug-cc-pVDZ System $\mathrm{Cl} 2 \mathrm{Ar} 1$

$\begin{array}{lllr}\mathrm{Cl} & 0.00000 & 0.00000 & -0.52502 \\ \mathrm{Cl} & 0.00000 & 0.00000 & -2.56500 \\ \mathrm{Ar} & 0.00000 & 0.00000 & 2.91836\end{array}$




\begin{tabular}{|c|c|c|c|}
\hline \multicolumn{4}{|c|}{ System $\mathrm{Cl} 2 \mathrm{Ar} 2$} \\
\hline $\mathrm{Cl}$ & 0.00000 & 1.01913 & 1.63537 \\
\hline $\mathrm{Cl}$ & -0.00000 & -1.01913 & 1.63537 \\
\hline $\mathrm{Ar}$ & 1.95915 & -0.00000 & -1.54452 \\
\hline $\mathrm{Ar}$ & -1.95915 & 0.00000 & -1.54452 \\
\hline \multicolumn{4}{|c|}{ System $\mathrm{Cl} 2 \mathrm{Ar} 3$} \\
\hline $\mathrm{Cl}$ & 1.09396 & -1.54082 & -0.03582 \\
\hline $\mathrm{Cl}$ & 2.59358 & -0.16051 & -0.00363 \\
\hline $\mathrm{Ar}$ & -0.41318 & 1.43920 & -1.90974 \\
\hline $\mathrm{Ar}$ & -2.65607 & -1.18121 & -0.02755 \\
\hline $\mathrm{Ar}$ & -0.41342 & 1.34881 & 1.97454 \\
\hline
\end{tabular}

\begin{tabular}{lrlc}
\multicolumn{5}{l}{ System $\mathrm{Cl} 2 \mathrm{Ar} 4$} \\
$\mathrm{Cl}$ & -1.77562 & 1.42307 & 0.00000 \\
$\mathrm{Cl}$ & 0.07629 & 2.27423 & 0.00000 \\
$\mathrm{Ar}$ & 0.07629 & -0.16598 & 2.99194 \\
$\mathrm{Ar}$ & 0.07629 & -0.16598 & -2.99194 \\
$\mathrm{Ar}$ & -1.01926 & -2.38219 & -0.00000 \\
$\mathrm{Ar}$ & 2.47160 & -0.77774 & -0.00000
\end{tabular}

System $\mathrm{Cl} 2 \mathrm{Ar} 5$

$\begin{array}{lrrr}\mathrm{Cl} & 0.07962 & -1.38291 & -0.78117 \\ \mathrm{Cl} & 1.81960 & -1.46782 & -1.84159 \\ \mathrm{Ar} & -0.72954 & 1.03319 & 2.15601 \\ \mathrm{Ar} & 2.81258 & -0.45121 & 1.74698 \\ \mathrm{Ar} & -2.31996 & 1.56959 & -1.35390 \\ \mathrm{Ar} & -3.02193 & -1.67110 & 0.70258 \\ \mathrm{Ar} & 1.46513 & 2.21188 & -0.77462\end{array}$

System $\mathrm{Cl} 2 \mathrm{Ar} 6$

$\begin{array}{llll}\mathrm{Cl} & -0.07455 & -0.00028 & 1.50874 \\ \mathrm{Cl} & -1.91351 & -0.00511 & 2.39048 \\ \mathrm{Ar} & -2.31255 & -1.94535 & -0.92504 \\ \mathrm{Ar} & 3.27259 & 0.00912 & 0.64430 \\ \mathrm{Ar} & 1.23709 & 3.22366 & -0.11761 \\ \mathrm{Ar} & -2.32335 & 1.93241 & -0.92525 \\ \mathrm{Ar} & 1.25518 & -3.21678 & -0.11756 \\ \mathrm{Ar} & 0.74865 & 0.00203 & -2.24144\end{array}$

System $\mathrm{Cl} 2 \mathrm{Ar} 7$

$\begin{array}{lrrr}\mathrm{Cl} & -0.08207 & -0.11016 & -1.23521 \\ \mathrm{Cl} & -0.69390 & -1.92743 & -1.92856 \\ \mathrm{Ar} & 3.73552 & 0.11002 & -0.97215 \\ \mathrm{Ar} & 1.47436 & 0.78753 & 2.13964 \\ \mathrm{Ar} & -1.89760 & 2.29976 & 1.17481 \\ \mathrm{Ar} & -1.70421 & -1.53341 & 1.83178\end{array}$




\begin{tabular}{|c|c|c|c|}
\hline $\mathrm{Ar}$ & 1.21515 & 3.08854 & -0.94698 \\
\hline $\mathrm{Ar}$ & 1.83580 & -2.76805 & 0.81228 \\
\hline $\mathrm{Ar}$ & -3.92614 & -0.06000 & -1.05139 \\
\hline \multicolumn{4}{|c|}{ System $\mathrm{Cl} 2 \mathrm{Ar} 8$} \\
\hline $\mathrm{Cl}$ & 0.00012 & 0.04452 & -0.84335 \\
\hline $\mathrm{Cl}$ & -0.00149 & -1.22650 & -2.43666 \\
\hline $\mathrm{Ar}$ & 3.25652 & -1.91502 & -0.47465 \\
\hline $\mathrm{Ar}$ & 0.00092 & 3.29151 & 0.57479 \\
\hline $\mathrm{Ar}$ & -3.25768 & -1.91332 & -0.47253 \\
\hline $\mathrm{Ar}$ & 3.25286 & 1.88396 & -1.19722 \\
\hline $\mathrm{Ar}$ & -1.92913 & 0.42315 & 2.27456 \\
\hline $\mathrm{Ar}$ & -3.25161 & 1.88487 & -1.19838 \\
\hline $\mathrm{Ar}$ & 1.92988 & 0.42185 & 2.27409 \\
\hline $\mathrm{Ar}$ & -0.00046 & -2.96069 & 1.31712 \\
\hline \multicolumn{4}{|c|}{ System $\mathrm{Cl} 2 \mathrm{Ar} 9$} \\
\hline $\mathrm{Cl}$ & 0.05519 & 0.01851 & -0.68887 \\
\hline $\mathrm{Cl}$ & -0.01360 & -1.53803 & -2.00318 \\
\hline $\mathrm{Ar}$ & 0.79245 & 0.22489 & 3.01695 \\
\hline $\mathrm{Ar}$ & -3.66407 & -1.53861 & -1.01945 \\
\hline $\mathrm{Ar}$ & -0.08851 & 3.21584 & 0.63890 \\
\hline $\mathrm{Ar}$ & -2.76466 & 0.77681 & 1.78660 \\
\hline $\mathrm{Ar}$ & 3.36129 & -0.00019 & -2.69775 \\
\hline $\mathrm{Ar}$ & -2.80198 & 2.08398 & -1.97747 \\
\hline $\mathrm{Ar}$ & 2.77179 & -2.17577 & 0.61004 \\
\hline $\mathrm{Ar}$ & -1.02385 & -2.80721 & 1.51389 \\
\hline $\mathrm{Ar}$ & 3.37826 & 1.65536 & 0.67079 \\
\hline \multicolumn{4}{|c|}{ System $\mathrm{Cl} 2 \mathrm{Ar} 10$} \\
\hline $\mathrm{Cl}$ & -0.00029 & -0.00004 & -0.35767 \\
\hline $\mathrm{Cl}$ & 0.00066 & -0.00018 & -2.39491 \\
\hline $\mathrm{Ar}$ & -0.00051 & 1.96071 & 2.63640 \\
\hline $\mathrm{Ar}$ & -3.73284 & 0.00025 & -1.92036 \\
\hline $\mathrm{Ar}$ & 3.73401 & -0.00039 & -1.91938 \\
\hline $\mathrm{Ar}$ & 1.94898 & -3.12049 & -0.58580 \\
\hline $\mathrm{Ar}$ & -1.94920 & -3.12065 & -0.58664 \\
\hline $\mathrm{Ar}$ & 1.94950 & 3.12033 & -0.58599 \\
\hline $\mathrm{Ar}$ & 3.12265 & -0.00005 & 1.75613 \\
\hline $\mathrm{Ar}$ & -1.94868 & 3.12083 & -0.58681 \\
\hline $\mathrm{Ar}$ & -0.00071 & -1.96046 & 2.63660 \\
\hline $\mathrm{Ar}$ & -3.12356 & 0.00014 & 1.75551 \\
\hline \multicolumn{4}{|c|}{ System Cl2Ar11 } \\
\hline $\mathrm{Cl}$ & 0.00050 & 0.00090 & -2.43602 \\
\hline $\mathrm{Cl}$ & 0.00007 & 0.00014 & -0.39776 \\
\hline $\mathrm{Ar}$ & 1.37645 & 3.04711 & 1.50890 \\
\hline $\mathrm{Ar}$ & 2.67834 & -2.43626 & -1.60341 \\
\hline
\end{tabular}




$\begin{array}{lrrr}\mathrm{Ar} & -2.47291 & 2.25046 & 1.50951 \\ \mathrm{Ar} & -0.73498 & 3.54574 & -1.60298 \\ \mathrm{Ar} & -2.90499 & -1.65625 & 1.50999 \\ \mathrm{Ar} & 0.67734 & -3.27448 & 1.50881 \\ \mathrm{Ar} & -1.49155 & -3.29836 & -1.60395 \\ \mathrm{Ar} & -0.00026 & -0.00010 & 3.14501 \\ \mathrm{Ar} & -3.59834 & 0.39687 & -1.60341 \\ \mathrm{Ar} & 3.32348 & -0.36781 & 1.51048 \\ \mathrm{Ar} & 3.14687 & 1.79210 & -1.60261\end{array}$

System $\mathrm{Cl} 2 \operatorname{Ar} 12$

$\begin{array}{llll}\mathrm{Cl} & 0.90881 & 0.00082 & -1.90669 \\ \mathrm{Cl} & -0.17060 & 0.00008 & -0.17783 \\ \mathrm{Ar} & -0.22826 & 3.31251 & 1.95685 \\ \mathrm{Ar} & -0.90360 & -3.32605 & -1.87325 \\ \mathrm{Ar} & -1.34888 & -0.00063 & 3.08900 \\ \mathrm{Ar} & -2.81326 & -0.00356 & -2.81741 \\ \mathrm{Ar} & -0.21993 & -3.31119 & 1.95758 \\ \mathrm{Ar} & 4.80836 & 0.00470 & -1.08397 \\ \mathrm{Ar} & 2.57931 & 0.00178 & 2.22711 \\ \mathrm{Ar} & 2.62691 & -2.94949 & -0.31911 \\ \mathrm{Ar} & 2.62056 & 2.95403 & -0.31788 \\ \mathrm{Ar} & -0.91013 & 3.32394 & -1.87451 \\ \mathrm{Ar} & -3.45203 & -1.88366 & 0.51283 \\ \mathrm{Ar} & -3.45626 & 1.87678 & 0.51148\end{array}$

System $\mathrm{Cl} 2 \mathrm{Ar} 13$

$\mathrm{Cl} \quad 0.00002$

$\begin{array}{ll}0.00002-0.0003 & -0.35759\end{array}$

$\begin{array}{llll}\text { Cl } & 0.00002 & -0.00005 & -2.39653\end{array}$

$\begin{array}{llll}\text { Ar } & 0.61147 & 3.61435 & 1.60432\end{array}$

$\begin{array}{llll}\text { Ar } & -2.39284 & -2.89214 & -1.66707\end{array}$

$\begin{array}{llll}\text { Ar } & 3.43549 & 1.27757 & 1.60445\end{array}$

$\begin{array}{llll}\text { Ar } & 1.30828 & -3.51837 & -1.66704\end{array}$

$\begin{array}{llll}\text { Ar } & -0.61143 & -3.61379 & 1.60444\end{array}$

$\begin{array}{llll}\text { Ar } & -3.43590 & -1.27761 & 1.60452\end{array}$

$\begin{array}{llll}\text { Ar } & 2.82436 & -2.33669 & 1.60450\end{array}$

$\begin{array}{llll}\text { Ar } & -2.82408 & 2.33647 & 1.60443\end{array}$

$\begin{array}{llll}\text { Ar } & -1.30826 & 3.51823 & -1.66708\end{array}$

$\begin{array}{llll}\text { Ar } & -3.70108 & 0.62617 & -1.66714\end{array}$

$\begin{array}{llll}\text { Ar } & 3.70113 & -0.62625 & -1.66698\end{array}$

$\begin{array}{llll}\text { Ar } & 0.00001 & 0.00007 & 2.97696\end{array}$

$\begin{array}{llll}\text { Ar } & 2.39282 & 2.89207 & -1.66720\end{array}$

System $\mathrm{Cl} 2 \mathrm{Ar} 14$

$\begin{array}{llll}\mathrm{Cl} & 0.96999 & 0.05030 & 0.31351 \\ \mathrm{Cl} & -0.96904 & 0.05006 & -0.31173 \\ \mathrm{Ar} & -1.01472 & -0.25149 & 3.46218 \\ \mathrm{Ar} & -2.90977 & 2.93078 & -1.70384\end{array}$




\begin{tabular}{|c|c|c|c|}
\hline $\mathrm{Ar}$ & -0.64665 & -3.32070 & -1.80448 \\
\hline 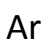 & 3.06671 & -2.71986 & -1.19170 \\
\hline $\mathrm{Ar}$ & 2.91588 & 2.92800 & 1.70206 \\
\hline $\mathrm{Ar}$ & -3.76509 & -0.81029 & -2.10501 \\
\hline $\mathrm{Ar}$ & 3.76633 & -0.81498 & 2.10476 \\
\hline $\mathrm{Ar}$ & -4.12233 & 0.97000 & 1.36679 \\
\hline $\mathrm{Ar}$ & -3.07272 & -2.71508 & 1.19269 \\
\hline $\mathrm{Ar}$ & -0.89826 & 3.16076 & 1.69501 \\
\hline $\mathrm{Ar}$ & 0.90328 & 3.15892 & -1.69629 \\
\hline $\mathrm{Ar}$ & 0.64009 & -3.31948 & 1.80536 \\
\hline $\mathrm{Ar}$ & 4.12363 & 0.96307 & -1.36812 \\
\hline $\mathrm{Ar}$ & 1.01275 & -0.25442 & -3.46112 \\
\hline \multicolumn{4}{|c|}{ System Cl2Ar15 } \\
\hline $\mathrm{Cl}$ & -0.99438 & -0.01972 & 0.22259 \\
\hline $\mathrm{Cl}$ & 0.99448 & -0.01953 & -0.22266 \\
\hline $\mathrm{Ar}$ & 1.87990 & -3.69659 & -0.03217 \\
\hline $\mathrm{Ar}$ & -3.68644 & -1.17027 & -2.19314 \\
\hline $\operatorname{Ar}$ & -3.78213 & 2.30768 & -0.61630 \\
\hline $\mathrm{Ar}$ & -2.48579 & 2.36249 & 2.89641 \\
\hline $\mathrm{Ar}$ & -3.99059 & -0.89542 & 1.64202 \\
\hline $\operatorname{Ar}$ & 3.78218 & 2.30758 & 0.61628 \\
\hline $\mathrm{Ar}$ & 3.68647 & -1.17040 & 2.19318 \\
\hline $\mathrm{Ar}$ & 3.99082 & -0.89554 & -1.64192 \\
\hline $\mathrm{Ar}$ & 1.09666 & 1.49841 & 3.17393 \\
\hline $\mathrm{Ar}$ & 0.00006 & 3.55146 & 0.00004 \\
\hline $\mathrm{Ar}$ & -1.88033 & -3.69672 & 0.03208 \\
\hline $\mathrm{Ar}$ & 2.48590 & 2.36216 & -2.89650 \\
\hline $\mathrm{Ar}$ & -0.04170 & -2.16312 & -3.01399 \\
\hline $\mathrm{Ar}$ & -1.09673 & 1.49862 & -3.17379 \\
\hline $\mathrm{Ar}$ & 0.04163 & -2.16329 & 3.01395 \\
\hline \multicolumn{4}{|c|}{ System $\mathrm{Cl} 2 \mathrm{Ar} 16$} \\
\hline $\mathrm{Cl}$ & 1.01919 & 0.02343 & 0.07115 \\
\hline $\mathrm{Cl}$ & -1.01924 & -0.02367 & 0.07109 \\
\hline $\mathrm{Ar}$ & 0.11528 & -2.00858 & -2.90538 \\
\hline $\mathrm{Ar}$ & -2.55831 & 2.04502 & 2.95501 \\
\hline $\mathrm{Ar}$ & -0.11503 & 2.01335 & -2.90203 \\
\hline $\mathrm{Ar}$ & 3.08956 & 3.43521 & -1.26481 \\
\hline $\mathrm{Ar}$ & 3.27230 & 0.14530 & -3.01194 \\
\hline $\mathrm{Ar}$ & -3.27202 & -0.14030 & -3.01253 \\
\hline $\mathrm{Ar}$ & 1.22287 & 1.48937 & 3.47068 \\
\hline $\mathrm{Ar}$ & 0.04787 & -3.63527 & 0.63656 \\
\hline $\mathrm{Ar}$ & -1.22367 & -1.49578 & 3.46783 \\
\hline $\mathrm{Ar}$ & -0.04753 & 3.63399 & 0.64292 \\
\hline $\mathrm{Ar}$ & -3.08950 & -3.43285 & -1.27018 \\
\hline $\mathrm{Ar}$ & 3.41010 & -2.83006 & -0.75224 \\
\hline $\mathrm{Ar}$ & 2.55796 & -2.05001 & 2.95189 \\
\hline
\end{tabular}




$\begin{array}{llll}\mathrm{Ar} & -3.40946 & 2.83159 & -0.74814 \\ \mathrm{Ar} & 4.30096 & 0.55063 & 0.80478 \\ \mathrm{Ar} & -4.30132 & -0.55138 & 0.80324\end{array}$

System $\mathrm{Cl} 2 \mathrm{Ar} 17$

$\begin{array}{llll}\text { Cl } & 1.02039 & -0.00014 & 0.00003\end{array}$

$\begin{array}{llll}\text { Cl } & -1.02037 & -0.00004 & 0.00003\end{array}$

$\begin{array}{llll}\text { Ar } & -4.67987 & 0.00024 & 0.00020\end{array}$

$\begin{array}{llll}\text { Ar } & -0.00020 & 2.40860 & -2.67299\end{array}$

$\begin{array}{llll}\text { Ar } & -0.00007 & 3.28711 & 1.46397\end{array}$

$\begin{array}{llll}\text { Ar } & 0.00015 & -3.52016 & 0.74694\end{array}$

$\begin{array}{llll}\text { Ar } & -0.00002 & -1.79870 & -3.11658\end{array}$

$\begin{array}{llll}\text { Ar } & 3.07143 & 1.66499 & 2.88546\end{array}$

$\begin{array}{llll}\text { Ar } & -3.07186 & 0.34897 & -3.31292\end{array}$

$\begin{array}{llll}\text { Ar } & -3.07205 & 3.25866 & -0.69164\end{array}$

$\begin{array}{llll}\text { Ar } & -3.07123 & 1.66488 & 2.88553\end{array}$

$\begin{array}{llll}\text { Ar } & 0.00014 & -0.37762 & 3.57859\end{array}$

$\begin{array}{llll}\text { Ar } & 3.07181 & 3.25883 & -0.69180\end{array}$

$\begin{array}{llll}\text { Ar } & -3.07161 & -2.22996 & 2.47493\end{array}$

$\begin{array}{llll}\text { Ar } & 3.07143 & 0.34923 & -3.31286\end{array}$

$\begin{array}{llll}\text { Ar } & 3.07185 & -2.22964 & 2.47464\end{array}$

$\begin{array}{llll}\text { Ar } & 3.07220 & -3.04274 & -1.35587\end{array}$

$\begin{array}{llll}\text { Ar } & -3.07195 & -3.04284 & -1.35559\end{array}$

$\begin{array}{llll}\text { Ar } & 4.67984 & 0.00031 & -0.00009\end{array}$

System $\mathrm{Cl} 2 \mathrm{Ar} 18$

$\begin{array}{llll}\text { Cl } & -0.29315 & 1.02033 & 0.00000\end{array}$

$\begin{array}{llll}\mathrm{Cl} & -0.29317 & -1.02043 & 0.00000\end{array}$

$\begin{array}{llll}\text { Ar } & -1.40169 & 0.00005 & 3.42191\end{array}$

$\begin{array}{llll}\text { Ar } & 3.05168 & 2.97170 & 0.00000\end{array}$

$\begin{array}{llll}\text { Ar } & -0.25553 & 4.66999 & 0.00000\end{array}$

$\begin{array}{llll}\text { Ar } & 5.62616 & -0.00003 & 0.00000\end{array}$

$\begin{array}{llll}\text { Ar } & -0.25575 & -4.67023 & 0.00000\end{array}$

$\begin{array}{llll}\text { Ar } & 0.71903 & -3.10083 & -3.18823\end{array}$

$\begin{array}{llll}\text { Ar } & 0.71903 & -3.10083 & 3.18823\end{array}$

$\begin{array}{llll}\text { Ar } & 2.53320 & -0.00004 & 2.22377\end{array}$

Ar $\quad-2.97858 \quad 3.07856 \quad 1.95050$

$\begin{array}{llll}\text { Ar } & -3.88939 & 0.00011 & 0.00000\end{array}$

$\begin{array}{llll}\text { Ar } & 3.05168 & -2.97182 & 0.00000\end{array}$

$\begin{array}{llll}\text { Ar } & 2.53320 & -0.00004 & -2.22377\end{array}$

$\begin{array}{llll}\text { Ar } & 0.71916 & 3.10081 & 3.18837\end{array}$

Ar $\quad-2.97858 \quad 3.07856 \quad-1.95050$

$\begin{array}{llll}\text { Ar } & 0.71916 & 3.10081 & -3.18837\end{array}$

$\begin{array}{llll}\text { Ar } & -2.97868 & -3.07836 & 1.95045\end{array}$

$\begin{array}{llll}\text { Ar } & -2.97868 & -3.07836 & -1.95045\end{array}$

$\begin{array}{llll}\text { Ar } & -1.40169 & 0.00005 & -3.42191\end{array}$

System $\mathrm{Cl} 2 \mathrm{Ar} 19$ 


\begin{tabular}{|c|c|c|c|}
\hline $\mathrm{Cl}$ & 0.00128 & -1.02026 & 0.17114 \\
\hline $\mathrm{Cl}$ & -0.00108 & 1.02051 & 0.17099 \\
\hline $\mathrm{Ar}$ & -1.93662 & -3.11107 & 2.87813 \\
\hline $\mathrm{Ar}$ & 5.62852 & 0.00605 & -1.64717 \\
\hline $\mathrm{Ar}$ & 0.00553 & -4.66095 & 0.14492 \\
\hline $\mathrm{Ar}$ & -3.17673 & 2.97407 & -0.85695 \\
\hline $\mathrm{Ar}$ & 0.00376 & -3.12158 & -3.18839 \\
\hline $\mathrm{Ar}$ & 3.16992 & 2.98135 & -0.85652 \\
\hline $\mathrm{Ar}$ & -3.16998 & -2.98172 & -0.85583 \\
\hline $\mathrm{Ar}$ & 2.00867 & 0.00183 & -2.81352 \\
\hline $\mathrm{Ar}$ & 1.94438 & -3.10687 & 2.87845 \\
\hline $\mathrm{Ar}$ & -5.62853 & -0.00686 & -1.64795 \\
\hline $\mathrm{Ar}$ & -1.94470 & 3.10856 & 2.87690 \\
\hline $\mathrm{Ar}$ & -3.37130 & -0.00345 & 1.41821 \\
\hline $\mathrm{Ar}$ & -0.00319 & 3.12022 & -3.18913 \\
\hline $\mathrm{Ar}$ & 3.37133 & 0.00392 & 1.41812 \\
\hline $\mathrm{Ar}$ & -0.00028 & 0.00100 & 3.76653 \\
\hline $\mathrm{Ar}$ & -2.00850 & -0.00275 & -2.81366 \\
\hline $\mathrm{Ar}$ & 3.17691 & -2.97457 & -0.85626 \\
\hline $\mathrm{Ar}$ & 1.93587 & 3.11171 & 2.87759 \\
\hline $\mathrm{Ar}$ & -0.00528 & 4.66087 & 0.14340 \\
\hline \multicolumn{4}{|c|}{ System Cl2Ar20 } \\
\hline $\mathrm{Cl}$ & 1.02061 & -0.00036 & 0.00001 \\
\hline $\mathrm{Cl}$ & -1.02091 & -0.00014 & 0.00022 \\
\hline $\mathrm{Ar}$ & -0.00021 & -3.70685 & 0.24699 \\
\hline $\mathrm{Ar}$ & 3.24823 & 0.24575 & 3.68729 \\
\hline $\operatorname{Ar}$ & 0.00032 & 3.70678 & -0.24705 \\
\hline $\mathrm{Ar}$ & 3.24815 & 3.31737 & 1.63114 \\
\hline $\mathrm{Ar}$ & 0.00034 & -1.63960 & 3.33373 \\
\hline $\mathrm{Ar}$ & -3.24785 & 3.07206 & -2.05708 \\
\hline $\operatorname{Ar}$ & 0.00048 & 2.06725 & 3.08665 \\
\hline $\mathrm{Ar}$ & 3.24777 & -3.07147 & 2.05686 \\
\hline $\mathrm{Ar}$ & 4.37779 & -0.00018 & -0.00030 \\
\hline $\mathrm{Ar}$ & -3.24720 & 3.31677 & 1.63128 \\
\hline $\mathrm{Ar}$ & -3.24777 & -3.07071 & 2.05686 \\
\hline $\mathrm{Ar}$ & -0.00026 & 1.63934 & -3.33345 \\
\hline $\mathrm{Ar}$ & -3.24738 & 0.24593 & 3.68862 \\
\hline $\mathrm{Ar}$ & -3.24821 & -3.31715 & -1.63119 \\
\hline $\mathrm{Ar}$ & 3.24724 & -0.24611 & -3.68880 \\
\hline $\mathrm{Ar}$ & -3.24799 & -0.24553 & -3.68706 \\
\hline $\mathrm{Ar}$ & 3.24775 & 3.07063 & -2.05712 \\
\hline $\mathrm{Ar}$ & -0.00046 & -2.06744 & -3.08665 \\
\hline $\mathrm{Ar}$ & -4.37793 & 0.00033 & 0.00030 \\
\hline $\mathrm{Ar}$ & 3.24748 & -3.31670 & -1.63125 \\
\hline
\end{tabular}

(d) $\mathrm{NH}_{3}\left(\mathrm{H}_{2} \mathrm{O}\right)_{n}(\mathrm{n}=1-20)$ systems, wB97XD/aug-cc-PVDZ System NH3(H2O)1 


$\begin{array}{llll}\mathrm{H} & -1.67796 & -0.50057 & -0.81656 \\ \mathrm{O} & 1.54132 & -0.10538 & 0.00001 \\ \mathrm{H} & 1.91092 & 0.77976 & 0.00001 \\ \mathrm{H} & 0.57444 & 0.02229 & -0.00002 \\ \mathrm{~N} & -1.36921 & 0.02010 & -0.00001 \\ \mathrm{H} & -1.87553 & 0.90108 & -0.00024 \\ \mathrm{H} & -1.67799 & -0.50016 & 0.81680\end{array}$

System NH3(H2O)2

$\begin{array}{llll}\mathrm{H} & -0.76444 & -1.17024 & -0.02477 \\ \mathrm{O} & 1.42855 & -0.95727 & 0.08456 \\ \mathrm{H} & 2.19960 & -1.11027 & -0.46466 \\ \mathrm{H} & 1.24278 & -0.00254 & 0.01707 \\ \mathrm{O} & 0.21885 & 1.55860 & -0.09962 \\ \mathrm{H} & 0.17911 & 2.15540 & 0.65059 \\ \mathrm{H} & -0.58104 & 0.98937 & -0.03373 \\ \mathrm{~N} & -1.59481 & -0.57579 & -0.00190 \\ \mathrm{H} & -2.13990 & -0.84576 & 0.81154 \\ \mathrm{H} & -2.15168 & -0.79611 & -0.82227\end{array}$

System NH3(H2O)3

$\begin{array}{cccc}\mathrm{O} & 1.51461 & -1.21984 & -0.01546 \\ \mathrm{H} & 1.86146 & -1.65034 & 0.76881 \\ \mathrm{H} & 0.55799 & -1.44345 & -0.03249 \\ \mathrm{H} & 1.96030 & 1.89568 & -0.63786 \\ \mathrm{O} & 1.34546 & 1.53210 & 0.00180 \\ \mathrm{H} & 1.50818 & 0.56633 & -0.00105 \\ \mathrm{O} & -1.18810 & -1.54574 & 0.00414 \\ \mathrm{H} & -1.45810 & -0.59133 & 0.00195 \\ \mathrm{H} & -1.64004 & -1.95095 & -0.73865 \\ \mathrm{H} & -0.65109 & 1.50519 & 0.00028 \\ \mathrm{~N} & -1.62519 & 1.19124 & 0.06435 \\ \mathrm{H} & -2.14180 & 1.65474 & -0.67765 \\ \mathrm{H} & -1.99633 & 1.54327 & 0.94238\end{array}$

\begin{tabular}{lrrr}
\multicolumn{4}{l}{ System NH3(H2O)4 } \\
$\mathrm{O}$ & 0.01084 & -1.45518 & 1.06445 \\
$\mathrm{H}$ & -0.35774 & -0.67383 & 1.50620 \\
$\mathrm{H}$ & -0.54014 & -1.49665 & 0.25299 \\
$\mathrm{O}$ & 2.20455 & -0.34384 & -0.00999 \\
$\mathrm{H}$ & 2.71233 & -0.98640 & -0.50905 \\
$\mathrm{H}$ & 1.48912 & -0.85640 & 0.44416 \\
$\mathrm{H}$ & -1.03100 & -0.10285 & -1.53248 \\
$\mathrm{O}$ & 0.43610 & 1.42212 & -0.99708 \\
$\mathrm{H}$ & 1.18535 & 0.83299 & -0.74519 \\
$\mathrm{H}$ & 0.82971 & 2.22702 & -1.33831 \\
$\mathrm{O}$ & -1.10971 & 1.17012 & 1.32244 \\
$\mathrm{H}$ & -0.94548 & 1.86063 & 1.96761
\end{tabular}




$\begin{array}{llll}\mathrm{H} & -0.58118 & 1.41125 & 0.53833 \\ \mathrm{~N} & -1.55534 & -0.92819 & -1.24137 \\ \mathrm{H} & -2.43682 & -0.59342 & -0.86120 \\ \mathrm{H} & -1.77096 & -1.47071 & -2.07203\end{array}$

System NH3(H2O) 5

$\begin{array}{llll}\mathrm{O} & 0.69161 & 1.67542 & 0.41495 \\ \mathrm{H} & 0.26593 & 1.11244 & 1.09111 \\ \mathrm{H} & 1.57097 & 1.25219 & 0.30226 \\ \mathrm{O} & -0.73212 & 0.64125 & -1.51916 \\ \mathrm{H} & -0.67929 & 1.10595 & -2.35607 \\ \mathrm{H} & -0.18001 & 1.15650 & -0.85966 \\ \mathrm{H} & 2.38734 & -0.81888 & -0.22628 \\ \mathrm{O} & 0.48246 & -1.70437 & -0.65524 \\ \mathrm{H} & 0.02984 & -0.97979 & -1.12851 \\ \mathrm{H} & 0.05889 & -2.51426 & -0.94710 \\ \mathrm{O} & -2.85528 & -0.11107 & 0.11905 \\ \mathrm{H} & -3.50229 & 0.57891 & 0.28058 \\ \mathrm{H} & -2.26177 & 0.24046 & -0.56938 \\ \mathrm{O} & -0.55825 & -0.43780 & 1.77920 \\ \mathrm{H} & -1.46260 & -0.35669 & 1.43149 \\ \mathrm{H} & -0.14577 & -1.05633 & 1.15789 \\ \mathrm{~N} & 2.94456 & -0.01274 & 0.05973 \\ \mathrm{H} & 3.40650 & -0.25835 & 0.93083 \\ \mathrm{H} & 3.67296 & 0.11954 & -0.63560\end{array}$

\begin{tabular}{llll}
\multicolumn{4}{l}{ System NH3(H2O) } \\
$\mathrm{H}$ & 2.46090 & -0.39441 & -0.77582 \\
$\mathrm{O}$ & -0.18642 & 2.18665 & -0.16732 \\
$\mathrm{H}$ & -0.30677 & 3.13341 & -0.25934 \\
$\mathrm{H}$ & -1.08661 & 1.78763 & -0.14607 \\
$\mathrm{O}$ & -1.08125 & -1.17131 & 1.51565 \\
$\mathrm{H}$ & -1.25000 & -1.64375 & 2.33235 \\
$\mathrm{H}$ & -0.26153 & -0.61611 & 1.66552 \\
$\mathrm{O}$ & 0.98628 & 0.41569 & 1.73302 \\
$\mathrm{H}$ & 1.76878 & 0.05548 & 1.24920 \\
$\mathrm{H}$ & 0.70542 & 1.17922 & 1.20295 \\
$\mathrm{O}$ & -2.45929 & 0.72570 & -0.05941 \\
$\mathrm{H}$ & -2.21432 & 0.14936 & 0.68464 \\
$\mathrm{H}$ & -2.38697 & 0.12477 & -0.81291 \\
$\mathrm{O}$ & 0.92904 & 0.17886 & -1.93998 \\
$\mathrm{H}$ & 0.22966 & -0.47497 & -1.78708 \\
$\mathrm{H}$ & 0.61960 & 0.96726 & -1.46878 \\
$\mathrm{O}$ & -1.19504 & -1.63959 & -1.17658 \\
$\mathrm{H}$ & -1.10663 & -1.72392 & -0.20676 \\
$\mathrm{H}$ & -1.30922 & -2.52616 & -1.52344 \\
$\mathrm{~N}$ & 3.00897 & -0.56764 & 0.07446 \\
$\mathrm{H}$ & 3.24553 & -1.55533 & 0.09303
\end{tabular}




\begin{tabular}{lrrr} 
H & 3.88282 & -0.05702 & -0.01173 \\
\multicolumn{4}{l}{} \\
System NH3(H2O)7 & \\
$\mathrm{O}$ & 1.90358 & -0.98100 & -1.42550 \\
$\mathrm{H}$ & 2.53506 & -1.59706 & -1.80094 \\
$\mathrm{H}$ & 1.80900 & -1.22505 & -0.47074 \\
$\mathrm{O}$ & -1.62188 & 1.48610 & -1.30321 \\
$\mathrm{H}$ & -2.34945 & 1.77574 & -1.85635 \\
$\mathrm{H}$ & -1.39284 & 0.56979 & -1.60062 \\
$\mathrm{O}$ & -1.40565 & -1.87208 & 0.80926 \\
$\mathrm{H}$ & -1.62932 & -0.94833 & 1.09681 \\
$\mathrm{H}$ & -2.04317 & -2.45681 & 1.22244 \\
$\mathrm{H}$ & 0.38024 & 2.05690 & -1.01999 \\
$\mathrm{H}$ & 1.70148 & 1.09388 & -1.12329 \\
$\mathrm{O}$ & -0.87158 & -1.02211 & -1.83696 \\
$\mathrm{H}$ & -1.12118 & -1.49546 & -1.02537 \\
$\mathrm{H}$ & 0.10208 & -1.06162 & -1.84972 \\
$\mathrm{O}$ & 1.36903 & -1.48647 & 1.14544 \\
$\mathrm{H}$ & 0.44548 & -1.79120 & 1.13410 \\
$\mathrm{H}$ & 1.32768 & -0.61302 & 1.57321 \\
$\mathrm{O}$ & 0.98902 & 1.19048 & 1.91468 \\
$\mathrm{H}$ & 1.42170 & 1.74813 & 2.56349 \\
$\mathrm{H}$ & 1.19896 & 1.56673 & 1.00806 \\
$\mathrm{O}$ & -1.72769 & 0.69644 & 1.38950 \\
$\mathrm{H}$ & -0.85177 & 0.96634 & 1.71759 \\
$\mathrm{H}$ & -1.79985 & 1.10993 & 0.50991 \\
$\mathrm{~N}$ & 1.32915 & 1.92728 & -0.66418 \\
$\mathrm{H}$ & 1.88321 & 2.71927 & -0.97499
\end{tabular}

\begin{tabular}{lrrr}
\multicolumn{4}{l}{ System NH3(H2O)8 } \\
$\mathrm{O}$ & -1.86785 & 1.98105 & 0.20198 \\
$\mathrm{H}$ & -1.07079 & 2.02107 & 0.77854 \\
$\mathrm{H}$ & -2.28044 & 2.84581 & 0.23324 \\
$\mathrm{O}$ & 2.74863 & -1.07448 & -0.38851 \\
$\mathrm{H}$ & 1.91857 & -1.42312 & -0.80123 \\
$\mathrm{H}$ & 3.47624 & -1.53247 & -0.81271 \\
$\mathrm{O}$ & -0.39304 & 0.81928 & -2.00207 \\
$\mathrm{H}$ & 0.49910 & 1.15931 & -1.78109 \\
$\mathrm{H}$ & -0.96396 & 1.31340 & -1.39658 \\
$\mathrm{O}$ & 0.45389 & 1.88837 & 1.56926 \\
$\mathrm{H}$ & 0.64850 & 0.93229 & 1.83708 \\
$\mathrm{H}$ & 0.69718 & 2.43746 & 2.31723 \\
$\mathrm{H}$ & 0.21579 & -1.28187 & 1.81768 \\
$\mathrm{H}$ & 1.77225 & -0.92940 & 1.43695 \\
$\mathrm{O}$ & 0.35422 & -1.76935 & -1.33191 \\
$\mathrm{H}$ & -0.20258 & -2.07410 & -0.59753 \\
$\mathrm{H}$ & -0.01509 & -0.90128 & -1.59616 \\
$\mathrm{O}$ & 2.01305 & 1.61856 & -0.87060
\end{tabular}




$\begin{array}{llll}\mathrm{H} & 1.61721 & 1.85430 & -0.01783 \\ \mathrm{H} & 2.42970 & 0.75347 & -0.71164 \\ \mathrm{O} & -1.35377 & -2.26925 & 0.90162 \\ \mathrm{H} & -1.80215 & -3.10101 & 1.06526 \\ \mathrm{H} & -2.02790 & -1.66499 & 0.51621 \\ \mathrm{O} & -2.94605 & -0.50225 & -0.38166 \\ \mathrm{H} & -2.68454 & 0.39111 & -0.09089 \\ \mathrm{H} & -2.65855 & -0.54356 & -1.29937 \\ \mathrm{~N} & 1.00869 & -0.68931 & 2.07306 \\ \mathrm{H} & 1.29796 & -0.96667 & 3.00650\end{array}$

System NH3(H2O) 9

$\begin{array}{lrrr}\mathrm{O} & 0.62306 & 2.40568 & -1.24052 \\ \mathrm{H} & -0.33184 & 2.17802 & -1.29974 \\ \mathrm{H} & 0.74903 & 3.19396 & -1.77193 \\ \mathrm{O} & -1.80992 & -1.54731 & 1.63865 \\ \mathrm{H} & -0.88971 & -1.86914 & 1.46184 \\ \mathrm{H} & -2.14028 & -2.07040 & 2.37098 \\ \mathrm{O} & 0.99023 & 1.94995 & 1.57315 \\ \mathrm{H} & 0.05116 & 1.71707 & 1.74631 \\ \mathrm{H} & 0.96930 & 2.30091 & 0.66958 \\ \mathrm{O} & -1.95877 & 1.60154 & -1.16489 \\ \mathrm{H} & -2.06055 & 0.60693 & -1.31891 \\ \mathrm{H} & -2.71706 & 2.02508 & -1.57151 \\ \mathrm{H} & -1.24603 & -1.47601 & -1.65379 \\ \mathrm{H} & -2.26944 & -1.37274 & -0.37930 \\ \mathrm{O} & 0.64645 & -2.32966 & 0.97546 \\ \mathrm{H} & 0.68076 & -2.40233 & 0.00547 \\ \mathrm{H} & 1.31759 & -1.65844 & 1.19401 \\ \mathrm{O} & -1.65620 & 1.25658 & 1.68966 \\ \mathrm{H} & -1.90364 & 1.46041 & 0.77464 \\ \mathrm{H} & -1.75224 & 0.28924 & 1.75837 \\ \mathrm{O} & 0.69907 & -2.09307 & -1.83949 \\ \mathrm{H} & 1.14607 & -2.70451 & -2.42751 \\ \mathrm{H} & 1.26995 & -1.28505 & -1.79837 \\ \mathrm{O} & 2.20670 & 0.07018 & -1.45083 \\ \mathrm{H} & 1.69904 & 0.89938 & -1.47321 \\ \mathrm{H} & 2.47447 & -0.02677 & -0.51983 \\ \mathrm{O} & 2.45880 & -0.21845 & 1.33452 \\ \mathrm{H} & 3.22650 & -0.18884 & 1.90812 \\ \mathrm{H} & 1.92665 & 0.60253 & 1.52474 \\ \mathrm{~N} & -2.13272 & -1.06910 & -1.34620 \\ \mathrm{H} & -2.86607 & -1.49909 & -1.90222\end{array}$

System NH3(H2O)10

$\begin{array}{lllr}\mathrm{O} & -0.92075 & -1.76923 & 0.30793 \\ \mathrm{H} & -1.87959 & -1.95304 & 0.29641 \\ \mathrm{H} & -0.77630 & -1.15511 & -0.43591\end{array}$




$\begin{array}{llll}\mathrm{O} & 2.75537 & 1.31668 & -0.40688 \\ \mathrm{H} & 2.68128 & 0.60130 & -1.06345 \\ \mathrm{H} & 1.97788 & 1.87841 & -0.55423 \\ \mathrm{O} & -3.68430 & -1.74096 & 0.16078 \\ \mathrm{H} & -3.61403 & -0.85596 & -0.25674 \\ \mathrm{H} & -4.23890 & -2.26311 & -0.42200 \\ \mathrm{O} & -0.34133 & 0.03006 & -1.79756 \\ \mathrm{H} & -0.08625 & 0.92883 & -1.52608 \\ \mathrm{H} & 0.49270 & -0.38145 & -2.08915 \\ \mathrm{O} & -3.02366 & 0.67901 & -0.94979 \\ \mathrm{H} & -2.26718 & 0.41311 & -1.49215 \\ \mathrm{H} & -2.62309 & 1.18521 & -0.22274 \\ \mathrm{O} & -1.53298 & 2.05254 & 1.09736 \\ \mathrm{H} & -1.95870 & 2.60554 & 1.75531 \\ \mathrm{H} & -1.06267 & 1.32259 & 1.60108 \\ \mathrm{O} & -0.30140 & 0.07322 & 2.25139 \\ \mathrm{H} & 0.67258 & 0.10798 & 2.24586 \\ \mathrm{H} & -0.53473 & -0.68061 & 1.67034 \\ \mathrm{O} & 0.25776 & 2.60625 & -0.82018 \\ \mathrm{H} & -0.39045 & 2.58000 & -0.08058 \\ \mathrm{H} & 0.03159 & 3.36592 & -1.36032 \\ \mathrm{O} & 2.48239 & 0.07862 & 1.95734 \\ \mathrm{H} & 3.09788 & 0.42581 & 2.60528 \\ \mathrm{H} & 2.64238 & 0.58444 & 1.12352 \\ \mathrm{H} & 2.29346 & -1.82081 & 1.01022 \\ \mathrm{H} & 0.96526 & -2.53684 & 0.37315 \\ \mathrm{O} & 2.23156 & -0.95616 & -1.98488 \\ \mathrm{H} & 2.76049 & -1.36326 & -2.67314 \\ \mathrm{H} & 2.20505 & -1.59715 & -1.20728 \\ \mathrm{~N} & 1.97562 & -2.43014 & 0.25451 \\ \mathrm{H} & 2.40069 & -3.34103 & 0.39706\end{array}$

\begin{tabular}{lccc}
\multicolumn{4}{l}{ System NH3(H2O) 11} \\
$\mathrm{O}$ & -2.59991 & 1.60877 & 1.52187 \\
$\mathrm{H}$ & -3.19274 & 2.29474 & 1.83514 \\
$\mathrm{H}$ & -2.63424 & 1.63386 & 0.53526 \\
$\mathrm{O}$ & -3.35685 & -1.32155 & -1.37495 \\
$\mathrm{H}$ & -4.06337 & -1.91574 & -1.63352 \\
$\mathrm{H}$ & -3.28598 & -1.37837 & -0.38960 \\
$\mathrm{O}$ & 2.48768 & -2.03348 & 1.10160 \\
$\mathrm{H}$ & 3.03849 & -2.64584 & 1.59213 \\
$\mathrm{H}$ & 2.74075 & -1.11672 & 1.38807 \\
$\mathrm{O}$ & 2.27058 & -1.27166 & -1.63268 \\
$\mathrm{H}$ & 1.31795 & -1.20731 & -1.81049 \\
$\mathrm{H}$ & 2.34129 & -1.72702 & -0.77704 \\
$\mathrm{H}$ & 1.72028 & 2.14725 & -1.28139 \\
$\mathrm{H}$ & 0.46124 & 1.22342 & -1.74716 \\
$\mathrm{O}$ & -0.16496 & -1.28357 & 1.07952
\end{tabular}




$\begin{array}{llll}\mathrm{H} & 0.03332 & -0.34608 & 1.30638 \\ \mathrm{H} & 0.66997 & -1.76428 & 1.21431 \\ \mathrm{O} & 0.22398 & 1.37263 & 1.43708 \\ \mathrm{H} & -0.66989 & 1.64203 & 1.70198 \\ \mathrm{H} & 0.33629 & 1.69497 & 0.50335 \\ \mathrm{O} & -0.55009 & -0.78334 & -1.56462 \\ \mathrm{H} & -1.37564 & -1.21549 & -1.83002 \\ \mathrm{H} & -0.45188 & -1.01912 & -0.61454 \\ \mathrm{O} & 2.92864 & 0.52901 & 1.62815 \\ \mathrm{H} & 2.06779 & 0.94929 & 1.78562 \\ \mathrm{H} & 3.21882 & 0.85864 & 0.75765 \\ \mathrm{O} & -2.92921 & -1.24456 & 1.27465 \\ \mathrm{H} & -1.98393 & -1.46057 & 1.36150 \\ \mathrm{H} & -2.97387 & -0.31327 & 1.54508 \\ \mathrm{O} & 3.52745 & 1.04317 & -1.06281 \\ \mathrm{H} & 4.41151 & 1.05959 & -1.43377 \\ \mathrm{H} & 3.11418 & 0.20106 & -1.36980 \\ \mathrm{O} & -2.50169 & 1.34872 & -1.15518 \\ \mathrm{H} & -1.64689 & 0.90307 & -1.26736 \\ \mathrm{H} & -3.11560 & 0.63301 & -1.38617 \\ \mathrm{~N} & 0.70952 & 2.04396 & -1.19604 \\ \mathrm{H} & 0.27059 & 2.84795 & -1.63451\end{array}$

System NH3(H2O) 12

$\begin{array}{lrrr}\mathrm{O} & -2.42651 & 0.07659 & -2.04798 \\ \mathrm{H} & -1.45873 & 0.12137 & -2.10614 \\ \mathrm{H} & -2.61658 & -0.82669 & -1.73985 \\ \mathrm{O} & 2.55478 & -2.32198 & 0.32431 \\ \mathrm{H} & 2.59716 & -1.79929 & 1.14256 \\ \mathrm{H} & 1.60850 & -2.32111 & 0.09401 \\ \mathrm{O} & 1.01804 & 2.84212 & -0.62183 \\ \mathrm{H} & 1.79284 & 2.35306 & -0.24338 \\ \mathrm{H} & 1.31894 & 3.74214 & -0.76044 \\ \mathrm{O} & -3.09411 & -0.84845 & 1.57307 \\ \mathrm{H} & -3.33480 & 0.01768 & 1.20242 \\ \mathrm{H} & -2.20431 & -0.71169 & 1.94434 \\ \mathrm{O} & -2.71674 & -2.30580 & -0.60531 \\ \mathrm{H} & -3.23655 & -3.11013 & -0.64740 \\ \mathrm{H} & -2.93823 & -1.85292 & 0.25197 \\ \mathrm{O} & -0.31636 & -0.27018 & 1.93749 \\ \mathrm{H} & -0.22308 & -0.72356 & 1.07519 \\ \mathrm{H} & -0.50828 & 0.67357 & 1.71304 \\ \mathrm{H} & 1.54376 & 0.00556 & -2.28188 \\ \mathrm{H} & 0.60658 & 1.29688 & -1.95846 \\ \mathrm{O} & 3.04062 & 1.38718 & 0.30415 \\ \mathrm{H} & 2.85901 & 0.85965 & 1.10090 \\ \mathrm{H} & 3.29183 & 0.73712 & -0.37616 \\ \mathrm{O} & -3.36929 & 1.51320 & 0.01444\end{array}$




$\begin{array}{cccc}\mathrm{H} & -4.13602 & 2.03714 & -0.22454 \\ \mathrm{H} & -3.06236 & 1.06412 & -0.80982 \\ \mathrm{O} & -0.03104 & -1.67636 & -0.44172 \\ \mathrm{H} & -0.89787 & -2.09677 & -0.57972 \\ \mathrm{H} & 0.08636 & -1.02629 & -1.16620 \\ \mathrm{O} & 2.31721 & -0.38441 & 2.39895 \\ \mathrm{H} & 2.59167 & -0.37528 & 3.31771 \\ \mathrm{H} & 1.33013 & -0.32466 & 2.39450 \\ \mathrm{O} & -0.95165 & 2.25021 & 1.25178 \\ \mathrm{H} & -1.81224 & 2.18301 & 0.80732 \\ \mathrm{H} & -0.32167 & 2.56771 & 0.57899 \\ \mathrm{O} & 3.38268 & -0.65627 & -1.58145 \\ \mathrm{H} & 4.20596 & -0.91622 & -1.99850 \\ \mathrm{H} & 3.16670 & -1.36265 & -0.92111 \\ \mathrm{~N} & 0.58378 & 0.35499 & -2.35761 \\ \mathrm{H} & 0.40376 & 0.45655 & -3.35332\end{array}$

System NH3(H2O) 13

$\begin{array}{llll}\mathrm{O} & 3.61150 & -0.25739 & 1.44034 \\ \mathrm{H} & 4.35877 & -0.49409 & 1.99304 \\ \mathrm{H} & 3.04894 & -1.06796 & 1.36440 \\ \mathrm{O} & -3.23581 & 1.61548 & -1.26644 \\ \mathrm{H} & -3.32176 & 1.56397 & -0.27509 \\ \mathrm{H} & -3.85276 & 2.28458 & -1.56795 \\ \mathrm{O} & -0.50397 & 1.34695 & -1.27796 \\ \mathrm{H} & -0.45974 & 0.36929 & -1.34307 \\ \mathrm{H} & -1.43468 & 1.58854 & -1.44014 \\ \mathrm{O} & 2.12560 & 2.16186 & 1.69672 \\ \mathrm{H} & 2.16858 & 2.44635 & 0.77096 \\ \mathrm{H} & 2.66163 & 1.35201 & 1.72304 \\ \mathrm{O} & 2.17252 & -2.02230 & -1.83602 \\ \mathrm{H} & 2.68531 & -1.17492 & -1.74454 \\ \mathrm{H} & 2.56725 & -2.51296 & -2.55897 \\ \mathrm{O} & -3.20469 & -1.20989 & -1.57387 \\ \mathrm{H} & -2.25181 & -1.38204 & -1.65678 \\ \mathrm{H} & -3.29589 & -0.24683 & -1.66688 \\ \mathrm{O} & -3.62441 & -1.53239 & 1.05696 \\ \mathrm{H} & -3.55962 & -1.50201 & 0.07125 \\ \mathrm{H} & -4.41632 & -2.03261 & 1.26195 \\ \mathrm{O} & -0.42337 & -1.37941 & -1.19674 \\ \mathrm{H} & -0.40042 & -1.58933 & -0.24255 \\ \mathrm{H} & 0.42525 & -1.69568 & -1.56025 \\ \mathrm{O} & -0.51013 & 1.37091 & 1.54908 \\ \mathrm{H} & 0.36684 & 1.70954 & 1.81988 \\ \mathrm{H} & -0.47502 & 1.41711 & 0.57731 \\ \mathrm{O} & 1.91021 & 2.50299 & -1.17535 \\ \mathrm{H} & 2.01556 & 3.28768 & -1.71656 \\ \mathrm{H} & 0.98867 & 2.18809 & -1.31101\end{array}$




$\begin{array}{llll}\mathrm{O} & 2.07940 & -2.39499 & 0.95978 \\ \mathrm{H} & 1.13376 & -2.21831 & 1.11803 \\ \mathrm{H} & 2.16008 & -2.49273 & -0.00366 \\ \mathrm{O} & 3.50576 & 0.20176 & -1.32548 \\ \mathrm{H} & 3.00222 & 1.03316 & -1.38294 \\ \mathrm{H} & 3.72551 & 0.11294 & -0.38128 \\ \mathrm{O} & -3.27114 & 1.24718 & 1.34896 \\ \mathrm{H} & -2.35804 & 1.38013 & 1.66121 \\ \mathrm{H} & -3.44394 & 0.29283 & 1.43403 \\ \mathrm{H} & -1.64287 & -1.80245 & 1.57466 \\ \mathrm{H} & -0.55395 & -0.60829 & 1.83332 \\ \mathrm{~N} & -0.64312 & -1.62147 & 1.69583 \\ \mathrm{H} & -0.39143 & -2.06181 & 2.57782\end{array}$

System NH3(H2O)14

$\begin{array}{llll}\mathrm{O} & 3.17843 & -0.85050 & 1.95340 \\ \mathrm{H} & 3.89084 & -1.14519 & 2.52413 \\ \mathrm{H} & 3.24551 & -1.37423 & 1.12509 \\ \mathrm{O} & 0.35429 & -0.92640 & 2.08525 \\ \mathrm{H} & 1.28992 & -0.99259 & 2.32848 \\ \mathrm{H} & 0.23323 & 0.00557 & 1.80162 \\ \mathrm{O} & 3.06148 & -2.15651 & -0.43715 \\ \mathrm{H} & 2.89781 & -1.44082 & -1.10722 \\ \mathrm{H} & 3.74709 & -2.71523 & -0.80914 \\ \mathrm{O} & -2.68291 & -2.34751 & -0.11182 \\ \mathrm{H} & -2.68414 & -1.94878 & 0.78547 \\ \mathrm{H} & -1.77806 & -2.67132 & -0.22942 \\ \mathrm{O} & -0.13289 & 1.84731 & -1.40563 \\ \mathrm{H} & 0.75657 & 2.21836 & -1.52817 \\ \mathrm{H} & -0.13223 & 0.98083 & -1.86820 \\ \mathrm{O} & 0.18289 & -2.51534 & -0.16046 \\ \mathrm{H} & 0.20619 & -1.95144 & 0.64061 \\ \mathrm{H} & 1.11197 & -2.73849 & -0.30619 \\ \mathrm{O} & -3.35873 & -0.52045 & -1.89611 \\ \mathrm{H} & -4.18636 & -0.77121 & -2.31013 \\ \mathrm{H} & -3.15727 & -1.22791 & -1.22623 \\ \mathrm{O} & 2.67986 & 1.90200 & 1.35742 \\ \mathrm{H} & 1.73094 & 1.95170 & 1.57012 \\ \mathrm{H} & 2.94119 & 0.99559 & 1.59126 \\ \mathrm{O} & -0.07243 & 1.64758 & 1.33109 \\ \mathrm{H} & -0.16047 & 1.69071 & 0.35518 \\ \mathrm{H} & -0.96136 & 1.84082 & 1.68106 \\ \mathrm{H} & -1.41735 & -0.58602 & -2.57229 \\ \mathrm{H} & -0.26803 & -1.43408 & -1.78426 \\ \mathrm{O} & -2.48426 & -1.10037 & 2.33251 \\ \mathrm{H} & -2.65990 & -0.15016 & 2.23278 \\ \mathrm{H} & -1.53576 & -1.15724 & 2.52091 \\ \mathrm{O} & -2.92761 & 1.97572 & -0.80106\end{array}$




$\begin{array}{lrrr}\mathrm{H} & -3.16184 & 1.12068 & -1.21373 \\ \mathrm{H} & -2.03338 & 2.16664 & -1.12285 \\ \mathrm{O} & 2.63863 & 2.34010 & -1.25579 \\ \mathrm{H} & 2.73240 & 2.22345 & -0.27326 \\ \mathrm{H} & 3.21622 & 3.06339 & -1.50625 \\ \mathrm{O} & 2.49427 & -0.26208 & -2.19659 \\ \mathrm{H} & 2.68617 & 0.65687 & -1.93276 \\ \mathrm{H} & 1.53544 & -0.27823 & -2.35063 \\ \mathrm{O} & -2.77520 & 1.71266 & 1.82947 \\ \mathrm{H} & -3.36788 & 2.31046 & 2.28861 \\ \mathrm{H} & -2.93868 & 1.83664 & 0.85758 \\ \mathrm{~N} & -0.40765 & -0.74870 & -2.53522 \\ \mathrm{H} & -0.17177 & -1.20746 & -3.41177\end{array}$

System NH3(H2O)15

$\begin{array}{llll}\mathrm{O} & -1.46721 & -1.39837 & -1.37251 \\ \mathrm{H} & -2.38317 & -1.58747 & -1.63993 \\ \mathrm{H} & -1.45795 & -1.51110 & -0.39660 \\ \mathrm{H} & 1.53357 & 1.92489 & -0.29248 \\ \mathrm{H} & 2.55311 & 1.79727 & -1.56050 \\ \mathrm{O} & 4.05314 & -1.45878 & -1.34814 \\ \mathrm{H} & 3.12062 & -1.47084 & -1.61636 \\ \mathrm{H} & 4.32544 & -0.52999 & -1.46916 \\ \mathrm{O} & 1.27010 & -0.94618 & -1.58985 \\ \mathrm{H} & 1.24418 & 0.02773 & -1.65665 \\ \mathrm{H} & 0.37257 & -1.25440 & -1.79794 \\ \mathrm{O} & 1.32092 & 1.32181 & 1.65492 \\ \mathrm{H} & 1.28066 & 0.35040 & 1.55379 \\ \mathrm{H} & 0.41891 & 1.58152 & 1.89062 \\ \mathrm{O} & -4.14276 & -1.47703 & 1.17756 \\ \mathrm{H} & -3.22266 & -1.66821 & 1.42231 \\ \mathrm{H} & -4.28213 & -0.55156 & 1.44292 \\ \mathrm{O} & -4.30049 & 1.34559 & 1.41425 \\ \mathrm{H} & -4.99526 & 1.90175 & 1.77177 \\ \mathrm{H} & -4.32098 & 1.45688 & 0.42963 \\ \mathrm{O} & 4.13714 & -1.69494 & 1.29802 \\ \mathrm{H} & 4.19451 & -1.70711 & 0.30720 \\ \mathrm{H} & 4.73900 & -2.36649 & 1.62376 \\ \mathrm{O} & 4.54856 & 1.27542 & -1.22611 \\ \mathrm{H} & 5.36339 & 1.73291 & -1.44142 \\ \mathrm{H} & 4.45256 & 1.32190 & -0.24224 \\ \mathrm{O} & -4.11230 & 1.40758 & -1.24568 \\ \mathrm{H} & -3.18540 & 1.60412 & -1.45507 \\ \mathrm{H} & -4.23573 & 0.47975 & -1.51110 \\ \mathrm{O} & 1.39693 & -1.40209 & 1.14871 \\ \mathrm{H} & 2.29937 & -1.68045 & 1.38532 \\ \mathrm{H} & 1.40237 & -1.32015 & 0.17359 \\ \mathrm{O} & -1.50305 & 1.36189 & 1.44824\end{array}$




$\begin{array}{llll}\mathrm{H} & -1.44704 & 1.46736 & 0.47612 \\ \mathrm{H} & -2.43694 & 1.52942 & 1.66060 \\ \mathrm{O} & -4.24350 & -1.41802 & -1.47939 \\ \mathrm{H} & -4.91890 & -1.98383 & -1.85825 \\ \mathrm{H} & -4.29582 & -1.52633 & -0.49421 \\ \mathrm{O} & 4.12230 & 1.15553 & 1.41220 \\ \mathrm{H} & 3.21584 & 1.40136 & 1.65970 \\ \mathrm{H} & 4.18401 & 0.20141 & 1.58482 \\ \mathrm{O} & -1.33803 & -1.38533 & 1.36852 \\ \mathrm{H} & -1.34969 & -0.41390 & 1.50472 \\ \mathrm{H} & -0.41703 & -1.64676 & 1.53824 \\ \mathrm{O} & -1.29012 & 1.32195 & -1.31335 \\ \mathrm{H} & -0.36895 & 1.54681 & -1.52970 \\ \mathrm{H} & -1.34122 & 0.34656 & -1.41421 \\ \mathrm{~N} & 1.56988 & 1.94034 & -1.31735 \\ \mathrm{H} & 1.35650 & 2.89204 & -1.60710\end{array}$

System NH3(H2O)16

$\begin{array}{llll}\mathrm{O} & 4.75506 & 0.03144 & 0.72311 \\ \mathrm{H} & 4.27060 & -0.67320 & 1.22658 \\ \mathrm{H} & 5.65086 & 0.05406 & 1.06461 \\ \mathrm{O} & 0.11323 & -1.47822 & -0.44963 \\ \mathrm{H} & -0.57112 & -1.53819 & -1.18725 \\ \mathrm{H} & 0.79128 & -2.16201 & -0.59662 \\ \mathrm{O} & -0.05926 & 3.08501 & -1.95034 \\ \mathrm{H} & 0.03922 & 3.83510 & -2.53934 \\ \mathrm{H} & 0.15504 & 3.40509 & -1.03435 \\ \mathrm{O} & 2.57760 & 1.75776 & 0.62670 \\ \mathrm{H} & 3.47451 & 1.39826 & 0.73621 \\ \mathrm{H} & 2.28377 & 1.44947 & -0.25460 \\ \mathrm{O} & 1.42806 & 0.74976 & -1.65847 \\ \mathrm{H} & 0.87477 & 1.49758 & -1.95025 \\ \mathrm{H} & 0.83625 & 0.06903 & -1.28939 \\ \mathrm{O} & -3.38349 & 0.09221 & 2.37011 \\ \mathrm{H} & -2.94005 & -0.72823 & 2.03475 \\ \mathrm{H} & -3.73674 & -0.12044 & 3.23602 \\ \mathrm{O} & 3.20286 & -1.81397 & 1.85853 \\ \mathrm{H} & 2.94653 & -2.38281 & 1.11299 \\ \mathrm{H} & 2.38605 & -1.33872 & 2.09034 \\ \mathrm{O} & 3.72530 & -0.86845 & -1.75620 \\ \mathrm{H} & 4.23370 & -0.48959 & -1.01834 \\ \mathrm{H} & 3.02704 & -0.21938 & -1.94301 \\ \mathrm{H} & -2.02536 & -0.33827 & -2.06000 \\ \mathrm{H} & -2.66521 & -1.84608 & -2.08707 \\ \mathrm{O} & -4.58159 & 0.44164 & -0.19821 \\ \mathrm{H} & -4.36023 & 0.46120 & 0.74718 \\ \mathrm{H} & -3.84275 & 0.91651 & -0.61947 \\ \mathrm{O} & 2.46130 & -2.93265 & -0.64977\end{array}$




$\begin{array}{llll}\mathrm{H} & 2.97433 & -2.24266 & -1.14482 \\ \mathrm{H} & 2.68754 & -3.78071 & -1.03608 \\ \mathrm{O} & 0.46321 & 3.62612 & 0.59376 \\ \mathrm{H} & -0.20350 & 3.12329 & 1.09282 \\ \mathrm{H} & 1.30432 & 3.18459 & 0.79377 \\ \mathrm{O} & -2.26285 & -2.04056 & 1.21945 \\ \mathrm{H} & -2.90508 & -2.25099 & 0.51655 \\ \mathrm{H} & -1.41291 & -1.91983 & 0.76942 \\ \mathrm{O} & -1.22082 & 1.62285 & 1.61218 \\ \mathrm{H} & -0.51917 & 0.98207 & 1.84536 \\ \mathrm{H} & -2.02004 & 1.29335 & 2.05982 \\ \mathrm{O} & -4.24467 & -2.16004 & -0.74728 \\ \mathrm{H} & -5.06462 & -2.65438 & -0.69917 \\ \mathrm{H} & -4.47929 & -1.21022 & -0.59794 \\ \mathrm{O} & -2.08931 & 1.39898 & -1.02046 \\ \mathrm{H} & -1.61806 & 2.09633 & -1.50177 \\ \mathrm{H} & -1.72148 & 1.43931 & -0.11430 \\ \mathrm{O} & 0.91234 & -0.10425 & 1.81789 \\ \mathrm{H} & 0.60016 & -0.56983 & 1.01866 \\ \mathrm{H} & 1.52852 & 0.57858 & 1.47926 \\ \mathrm{~N} & -1.81744 & -1.31487 & -2.28567 \\ \mathrm{H} & -1.63257 & -1.37532 & -3.28188\end{array}$

System $\mathrm{NH} 3(\mathrm{H} 2 \mathrm{O}) 17$

$\begin{array}{lrrr}\mathrm{O} & 2.00983 & 1.22558 & -1.37037 \\ \mathrm{H} & 2.96730 & 1.19186 & -1.55544 \\ \mathrm{H} & 1.70159 & 0.29588 & -1.37320 \\ \mathrm{O} & -1.29352 & -1.43752 & 1.17666 \\ \mathrm{H} & -0.35301 & -1.58877 & 1.40410 \\ \mathrm{H} & -1.32089 & -1.40622 & 0.19878 \\ \mathrm{O} & 4.73123 & 0.55544 & 1.21464 \\ \mathrm{H} & 3.89400 & 0.94624 & 1.52458 \\ \mathrm{H} & 4.64801 & -0.40296 & 1.36381 \\ \mathrm{O} & 4.32676 & -2.23290 & 1.10742 \\ \mathrm{H} & 4.25332 & -2.24756 & 0.12200 \\ \mathrm{H} & 4.96350 & -2.91041 & 1.34200 \\ \mathrm{O} & -0.08411 & 3.07850 & 1.59188 \\ \mathrm{H} & -0.85842 & 2.50314 & 1.70668 \\ \mathrm{H} & -0.08049 & 3.29757 & 0.64821 \\ \mathrm{O} & 0.13695 & 3.15282 & -1.33443 \\ \mathrm{H} & 0.32336 & 3.90135 & -1.90433 \\ \mathrm{H} & 0.88001 & 2.51903 & -1.44922 \\ \mathrm{O} & 1.23571 & -1.39481 & -1.09545 \\ \mathrm{H} & 1.19109 & -1.53717 & -0.13373 \\ \mathrm{H} & 0.32044 & -1.49122 & -1.41967 \\ \mathrm{O} & -2.04449 & 1.45529 & -1.21131 \\ \mathrm{H} & -1.37552 & 2.12789 & -1.43152 \\ \mathrm{H} & -2.06577 & 1.43361 & -0.22967\end{array}$




$\begin{array}{llll}\mathrm{O} & -3.99923 & -2.15415 & 1.13224 \\ \mathrm{H} & -3.06049 & -2.07971 & 1.37304 \\ \mathrm{H} & -4.39108 & -1.30279 & 1.39236 \\ \mathrm{O} & 2.10658 & 1.41190 & 1.44810 \\ \mathrm{H} & 1.40824 & 2.04623 & 1.70827 \\ \mathrm{H} & 2.04852 & 1.40385 & 0.47650 \\ \mathrm{O} & 3.95635 & -1.97636 & -1.53801 \\ \mathrm{H} & 4.28889 & -1.07681 & -1.69532 \\ \mathrm{H} & 2.99020 & -1.90251 & -1.60454 \\ \mathrm{O} & 4.71252 & 0.75873 & -1.41660 \\ \mathrm{H} & 5.46945 & 1.22674 & -1.77340 \\ \mathrm{H} & 4.81590 & 0.74623 & -0.42538 \\ \mathrm{O} & -2.16034 & 1.14896 & 1.50662 \\ \mathrm{H} & -1.81093 & 0.23456 & 1.54048 \\ \mathrm{H} & -3.11545 & 1.06646 & 1.67407 \\ \mathrm{O} & -4.72612 & 0.66029 & -1.28623 \\ \mathrm{H} & -4.60577 & -0.26585 & -1.55553 \\ \mathrm{H} & -3.87067 & 1.08607 & -1.46817 \\ \mathrm{O} & -4.05655 & -2.08956 & -1.52964 \\ \mathrm{H} & -4.08728 & -2.22210 & -0.54638 \\ \mathrm{H} & -4.52836 & -2.82417 & -1.92639 \\ \mathrm{O} & -1.44605 & -1.17802 & -1.57413 \\ \mathrm{H} & -1.63549 & -0.21473 & -1.58728 \\ \mathrm{H} & -2.29083 & -1.61931 & -1.77407 \\ \mathrm{H} & 2.37572 & -1.93927 & 1.71485 \\ \mathrm{H} & 1.66649 & -0.47638 & 1.91435 \\ \mathrm{O} & -4.87018 & 0.52543 & 1.37148 \\ \mathrm{H} & -5.66724 & 0.90823 & 1.74273 \\ \mathrm{H} & -4.92241 & 0.64309 & 0.38783 \\ \mathrm{~N} & 1.47587 & -1.48491 & 1.89097 \\ \mathrm{H} & 1.22017 & -1.75271 & 2.83886\end{array}$

\begin{tabular}{lrrl}
\multicolumn{4}{l}{ System NH3(H2O) 18} \\
$\mathrm{O}$ & 3.94075 & -0.20435 & 1.21315 \\
$\mathrm{H}$ & 3.49003 & -1.01625 & 1.50765 \\
$\mathrm{H}$ & 3.31476 & 0.51211 & 1.43293 \\
$\mathrm{O}$ & -0.03248 & 1.09265 & 3.31143 \\
$\mathrm{H}$ & 0.74228 & 1.39937 & 2.77521 \\
$\mathrm{H}$ & 0.00672 & 1.55162 & 4.15287 \\
$\mathrm{O}$ & 2.14773 & -2.56292 & -1.06763 \\
$\mathrm{H}$ & 2.82737 & -1.90891 & -1.30426 \\
$\mathrm{H}$ & 2.24905 & -2.70521 & -0.11024 \\
$\mathrm{O}$ & -1.22506 & 3.84458 & -0.15857 \\
$\mathrm{H}$ & -0.26314 & 3.77988 & -0.31150 \\
$\mathrm{H}$ & -1.62750 & 3.47517 & -0.95332 \\
$\mathrm{O}$ & -1.97945 & -1.84077 & 1.36862 \\
$\mathrm{H}$ & -2.13193 & -2.31886 & 0.53373 \\
$\mathrm{H}$ & -2.76627 & -1.29146 & 1.51173
\end{tabular}




$\begin{array}{llll}\mathrm{O} & 1.81804 & 1.56146 & 1.50437 \\ \mathrm{H} & 1.29479 & 0.91535 & 0.93067 \\ \mathrm{H} & 1.83027 & 2.38159 & 0.98267 \\ \mathrm{O} & -0.19408 & -2.35028 & -2.53861 \\ \mathrm{H} & -0.17511 & -1.42180 & -2.82218 \\ \mathrm{H} & 0.63501 & -2.49224 & -2.04153 \\ \mathrm{H} & -0.39870 & -0.61517 & 0.56127 \\ \mathrm{H} & -0.00347 & 0.24192 & -0.76258 \\ \mathrm{O} & 2.09303 & 1.64552 & -2.29633 \\ \mathrm{H} & 1.31502 & 1.19782 & -2.67064 \\ \mathrm{H} & 2.70994 & 0.94418 & -2.01536 \\ \mathrm{O} & -4.09125 & -0.60467 & -1.32415 \\ \mathrm{H} & -3.60112 & -1.44600 & -1.33947 \\ \mathrm{H} & -4.31042 & -0.45816 & -0.39016 \\ \mathrm{O} & -2.39813 & -2.85880 & -1.17947 \\ \mathrm{H} & -2.67498 & -3.74913 & -1.40462 \\ \mathrm{H} & -1.56519 & -2.69087 & -1.69813 \\ \mathrm{O} & 3.84962 & -0.34535 & -1.41478 \\ \mathrm{H} & 4.72904 & -0.36372 & -1.79788 \\ \mathrm{H} & 3.97180 & -0.25287 & -0.42963 \\ \mathrm{O} & -2.14296 & 1.25700 & -1.43766 \\ \mathrm{H} & -2.90089 & 0.62806 & -1.50670 \\ \mathrm{H} & -1.95259 & 1.27358 & -0.48478 \\ \mathrm{O} & -0.29549 & 0.44537 & -3.17893 \\ \mathrm{H} & -1.01020 & 0.78635 & -2.58433 \\ \mathrm{H} & -0.56526 & 0.65790 & -4.07529 \\ \mathrm{O} & 0.20630 & -1.74526 & 3.02177 \\ \mathrm{H} & -0.60522 & -1.90241 & 2.50188 \\ \mathrm{H} & 0.16625 & -0.79933 & 3.23883 \\ \mathrm{H} & 2.39766 & -2.50631 & 1.74414 \\ \mathrm{H} & 1.57082 & -2.22098 & 2.21871 \\ \mathrm{H} & 2.74524 & -3.24009 & 2.25548 \\ \mathrm{O} & -1.98437 & 1.74359 & 1.39626 \\ \mathrm{H} & -1.73159 & 2.61913 & 1.03412 \\ \mathrm{H} & -1.32991 & 1.51737 & 2.08223 \\ \mathrm{O} & -4.06126 & 0.07025 & 1.44117 \\ \mathrm{H} & -4.73841 & 0.25737 & 2.09361 \\ & -3.42143 & 0.81687 & 1.47210 \\ \mathrm{H} & 2.53538 & 3.50725 & -0.50777 \\ \mathrm{H} & -.75900 & 2.83526 & -1.20575 \\ \mathrm{H} & -0.97592 & -0.28332\end{array}$

System NH3(H2O) 19

$\begin{array}{llll}\mathrm{O} & -0.08585 & 0.18069 & -0.94215 \\ \mathrm{H} & -0.13131 & 0.22063 & 0.02622 \\ \mathrm{H} & -0.88227 & 0.67673 & -1.23992\end{array}$




\begin{tabular}{|c|c|c|c|}
\hline 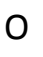 & 2.41914 & 1.10886 & -1.47431 \\
\hline H & 1.49344 & 0.79645 & -1.42201 \\
\hline & 2.34746 & 2.06064 & -1.66626 \\
\hline & 1.77049 & 3.80828 & -1.54148 \\
\hline & 2.09730 & 4.52757 & -2.08538 \\
\hline & 0.78094 & 3.85759 & -1.58102 \\
\hline b & -2.35530 & 1.57079 & -1.35816 \\
\hline - & -2.42771 & 1.61654 & -0.37999 \\
\hline $\mathrm{H}$ & -3.06623 & 0.96498 & -1.63745 \\
\hline 0 & -0.37419 & -2.83690 & 1.29166 \\
\hline H & -1.30655 & -2.95504 & 1.53756 \\
\hline $\mathrm{H}$ & -0.38157 & -2.78197 & 0.31528 \\
\hline 0 & -3.17212 & -2.80150 & 1.46876 \\
\hline $\mathrm{H}$ & -3.52262 & -1.87499 & 1.48135 \\
\hline H & -3.78658 & -3.32732 & 1.98444 \\
\hline b & -4.16173 & -0.33165 & 1.23384 \\
\hline $\mathrm{H}$ & -4.34382 & -0.27340 & 0.28065 \\
\hline 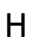 & -3.58383 & 0.42604 & 1.42745 \\
\hline o & 2.73908 & 1.02300 & 1.27720 \\
\hline-1 & 3.32283 & 0.28092 & 1.50910 \\
\hline$y$ & 2.71876 & 1.03144 & 0.29993 \\
\hline H & 0.98843 & 0.34373 & 1.90719 \\
\hline $\mathrm{H}$ & -0.02111 & -0.97491 & 1.91448 \\
\hline $\mathrm{O}$ & 1.74532 & 3.62674 & 1.29955 \\
\hline H & 1.92928 & 3.76804 & 0.35536 \\
\hline $\mathrm{H}$ & 2.15620 & 2.76920 & 1.50590 \\
\hline 0 & 2.40199 & -3.33057 & -1.63406 \\
\hline H & 2.99396 & -2.53261 & -1.64148 \\
\hline $\mathrm{H}$ & 2.79237 & -3.96240 & -2.24096 \\
\hline 0 & 3.96946 & -1.20392 & -1.41000 \\
\hline $\mathrm{H}$ & 4.18778 & -1.19658 & -0.46249 \\
\hline $\mathrm{H}$ & 3.53949 & -0.34730 & -1.58001 \\
\hline 0 & -4.22131 & -0.44712 & -1.61072 \\
\hline $\mathrm{H}$ & -4.98782 & -0.54214 & -2.17928 \\
\hline $\mathrm{H}$ & -3.75577 & -1.32535 & -1.61669 \\
\hline 0 & -0.88067 & 3.97374 & -1.37801 \\
\hline $\mathrm{H}$ & -1.02269 & 4.11658 & -0.42654 \\
\hline $\mathrm{H}$ & -1.44622 & 3.21679 & $-1.6024 \mathrm{C}$ \\
\hline 0 & -0.86345 & 4.06617 & 1.46420 \\
\hline $\mathrm{H}$ & 0.11092 & 3.87521 & 1.49567 \\
\hline $\mathrm{H}$ & -1.00062 & 4.84722 & 2.00383 \\
\hline O & 2.35751 & -3.39339 & 1.21613 \\
\hline $\mathrm{H}$ & 2.34850 & -3.56846 & 0.26004 \\
\hline $\mathrm{H}$ & 1.42026 & -3.29794 & 1.45846 \\
\hline $\mathrm{O}$ & 4.09939 & -1.39733 & 1.42617 \\
\hline $\mathrm{H}$ & 4.85126 & -1.70150 & 1.93792 \\
\hline $\mathrm{H}$ & 3.43271 & -2.13169 & 1.44738 \\
\hline 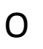 & -2.15841 & 1.62159 & 1.3582 \\
\hline
\end{tabular}




$\begin{array}{llll}\mathrm{H} & -1.37847 & 1.05575 & 1.58246 \\ \mathrm{H} & -1.86096 & 2.52965 & 1.54865 \\ \mathrm{O} & -2.99969 & -2.78672 & -1.37300 \\ \mathrm{H} & -3.07612 & -2.97762 & -0.42268 \\ \mathrm{H} & -2.04399 & -2.80795 & -1.55771 \\ \mathrm{O} & -0.23454 & -2.50415 & -1.45023 \\ \mathrm{H} & -0.12973 & -1.53343 & -1.40277 \\ \mathrm{H} & 0.64257 & -2.86100 & -1.68195 \\ \mathrm{~N} & 0.02568 & 0.03592 & 2.08054 \\ \mathrm{H} & -0.13923 & 0.16772 & 3.07524\end{array}$

(e) $F^{-}\left(\mathrm{H}_{2} \mathrm{O}\right)_{\mathrm{n}}(\mathrm{n}=1-20)$ systems, B3LYP/aug-cc-pVDZ

$\begin{array}{llll}F & 0.04802 & 1.24980 & 0.00000\end{array}$

$\begin{array}{llll}0 & 0.04802 & -1.21319 & 0.00000\end{array}$

$\begin{array}{llll}\mathrm{H} & -0.89891 & -1.38856 & 0.00000\end{array}$

$\begin{array}{llll}\text { H } & 0.08262 & -0.15415 & 0.00000\end{array}$

System F-(H2O)2

$\begin{array}{llll}F & 0.00000 & -0.89656 & 0.00000\end{array}$

$\begin{array}{llll}O & -2.18036 & 0.39705 & -0.07178\end{array}$

$\begin{array}{llll}\mathrm{H} & -2.04105 & 1.00538 & 0.66149\end{array}$

$\begin{array}{llll}\mathrm{H} & -1.31611 & -0.14725 & -0.07459\end{array}$

$\begin{array}{llll}\mathrm{O} & 2.18035 & 0.39705 & 0.07178\end{array}$

$\begin{array}{llll}\mathrm{H} & 1.31610 & -0.14724 & 0.07460\end{array}$

$\begin{array}{llll}\text { H } & 2.04109 & 1.00531 & -0.66156\end{array}$

System F-( $\mathrm{H} 2 \mathrm{O}) 3$

$\begin{array}{llll}\mathrm{F} & -0.00043 & 0.00023 & 0.73406 \\ \mathrm{O} & -2.39562 & 0.34441 & -0.20600 \\ \mathrm{H} & -2.23929 & 1.12142 & -0.75337 \\ \mathrm{H} & -1.49169 & 0.17756 & 0.19799 \\ \mathrm{O} & 0.89957 & -2.24617 & -0.20583 \\ \mathrm{H} & 0.14823 & -2.49982 & -0.75259 \\ \mathrm{H} & 0.59198 & -1.37994 & 0.19808 \\ \mathrm{O} & 1.49654 & 1.90162 & -0.20589 \\ \mathrm{H} & 0.89956 & 1.20268 & 0.19808 \\ \mathrm{H} & 2.09117 & 1.37722 & -0.75290\end{array}$

System F-( $\mathrm{H} 2 \mathrm{O}) 4$

F $\quad 0.00001 \quad-0.00002 \quad-0.03093$

$\begin{array}{llll}\mathrm{O} & 2.10420 & 1.17200 & -1.06927\end{array}$

$\begin{array}{llll}\mathrm{H} & 1.26768 & 0.73205 & -0.74988\end{array}$

$\begin{array}{llll}\mathrm{H} & 2.11694 & 1.99991 & -0.57721\end{array}$

$\begin{array}{llll}\mathrm{H} & -2.10426 & -1.17202 & -1.06923\end{array}$

$\begin{array}{llll}\mathrm{H} & -2.11703 & -1.99989 & -0.57710\end{array}$

$\begin{array}{llll}\mathrm{H} & -1.26773 & -0.73207 & -0.74985\end{array}$

$\begin{array}{llll}\mathrm{O} & 2.06806 & -1.30655 & 1.08561\end{array}$

$\begin{array}{llll}\text { H } & 2.69824 & -0.80051 & 0.55675\end{array}$ 


$\begin{array}{rrrr}\mathrm{H} & 1.21266 & -0.91943 & 0.77853 \\ \mathrm{O} & -2.06800 & 1.30659 & 1.08561 \\ \mathrm{H} & -1.21261 & 0.91944 & 0.77853 \\ \mathrm{H} & -2.69820 & 0.80056 & 0.55675\end{array}$

System F-(H2O) 5

$\begin{array}{llll}\text { F } & 0.81448 & -0.10302 & -0.04577 \\ \mathrm{O} & 3.14798 & -1.13584 & -0.78051 \\ \mathrm{H} & 2.94071 & -2.07629 & -0.77520 \\ \mathrm{H} & 2.28916 & -0.71320 & -0.52021 \\ \mathrm{O} & -0.83254 & -1.72400 & 1.18007 \\ \mathrm{H} & -0.92272 & -1.29847 & 2.03995 \\ \mathrm{H} & -0.17081 & -1.14814 & 0.70452 \\ \mathrm{O} & -3.08895 & -0.58430 & -0.36062 \\ \mathrm{H} & -2.53634 & 0.11015 & -0.76636 \\ \mathrm{H} & -2.43805 & -1.08640 & 0.16372 \\ \mathrm{O} & -1.04719 & 1.30102 & -1.32840 \\ \mathrm{H} & -0.32896 & 0.70830 & -0.99156 \\ \mathrm{H} & -0.98180 & 2.04904 & -0.72046 \\ \mathrm{O} & 0.85283 & 2.20314 & 1.22874 \\ \mathrm{H} & 1.68983 & 2.59715 & 0.96083 \\ \mathrm{H} & 0.87177 & 1.30494 & 0.80244\end{array}$

System F-(H2O)6

$\begin{array}{lrrr}\mathrm{F} & -0.72868 & -0.36808 & -0.20949 \\ \mathrm{O} & 2.78547 & 0.54915 & -0.01745 \\ \mathrm{H} & 2.16132 & 1.00188 & -0.62053 \\ \mathrm{H} & 2.24106 & 0.39090 & 0.77679 \\ \mathrm{O} & 0.80694 & -0.37651 & 1.96286 \\ \mathrm{H} & 0.16936 & -0.37729 & 1.20351 \\ \mathrm{H} & 1.16960 & -1.27099 & 1.92008 \\ \mathrm{O} & -1.62030 & 1.99432 & 0.69420 \\ \mathrm{H} & -1.37292 & 1.09732 & 0.36109 \\ \mathrm{H} & -1.20708 & 2.02881 & 1.56488 \\ \mathrm{O} & 0.57426 & 1.53269 & -1.57158 \\ \mathrm{H} & 0.08527 & 0.74563 & -1.22833 \\ \mathrm{H} & 0.12990 & 2.25482 & -1.10640 \\ \mathrm{O} & -3.25832 & -1.31159 & -0.38601 \\ \mathrm{H} & -3.70652 & -0.50990 & -0.09412 \\ \mathrm{H} & -2.30696 & -1.05447 & -0.35875 \\ \mathrm{O} & 1.51534 & -2.12906 & -0.60333 \\ \mathrm{H} & 0.64229 & -1.69426 & -0.60159 \\ \mathrm{H} & 2.12565 & -1.37170 & -0.56074\end{array}$

System F-(H2O)7

$\begin{array}{cccc}\mathrm{F} & 1.62961 & -0.37220 & -0.21746 \\ \mathrm{O} & 4.16653 & -0.07669 & 0.53621 \\ \mathrm{H} & 3.24290 & -0.22470 & 0.21132\end{array}$




$\begin{array}{rrrr}\mathrm{H} & 4.07037 & -0.10152 & 1.49429 \\ \mathrm{O} & -1.25340 & 2.01141 & 0.43228 \\ \mathrm{H} & -0.55895 & 2.09700 & -0.25474 \\ \mathrm{H} & -0.86601 & 1.33957 & 1.04447 \\ \mathrm{O} & -3.40081 & 0.57825 & -0.36922 \\ \mathrm{H} & -4.08099 & 0.82864 & 0.26558 \\ \mathrm{H} & -2.62308 & 1.15609 & -0.13625 \\ \mathrm{O} & 0.94384 & 1.84991 & -1.35373 \\ \mathrm{H} & 1.23909 & 0.98149 & -0.95059 \\ \mathrm{H} & 1.67658 & 2.44927 & -1.17513 \\ \mathrm{O} & 0.07510 & -2.12432 & -1.36388 \\ \mathrm{H} & 0.72846 & -1.50218 & -0.93645 \\ \mathrm{H} & -0.17312 & -1.67196 & -2.17794 \\ \mathrm{O} & -2.04748 & -1.85202 & 0.52136 \\ \mathrm{H} & -1.37648 & -2.03520 & -0.17025 \\ \mathrm{H} & -2.57125 & -1.10972 & 0.16337 \\ \mathrm{O} & -0.13031 & -0.11607 & 1.82865 \\ \mathrm{H} & 0.59016 & -0.23540 & 1.16616 \\ \mathrm{H} & -0.79200 & -0.78538 & 1.55988\end{array}$

System F-( $\mathrm{H} 2 \mathrm{O}) 8$

$\begin{array}{llll}\mathrm{F} & 0.06976 & -1.13243 & -1.10062 \\ \mathrm{O} & 2.25129 & -2.24550 & -0.22502 \\ \mathrm{H} & 2.83177 & -2.22970 & -0.99429 \\ \mathrm{H} & 1.38057 & -1.90494 & -0.57912 \\ \mathrm{O} & -3.62214 & -0.79919 & 0.58832 \\ \mathrm{H} & -2.69882 & -0.94752 & 0.93366 \\ \mathrm{H} & -3.90395 & -1.66959 & 0.28557 \\ \mathrm{O} & 2.87210 & 0.25876 & 1.04161 \\ \mathrm{H} & 2.68280 & -0.66227 & 0.76509 \\ \mathrm{H} & 2.05547 & 0.56717 & 1.47329 \\ \mathrm{O} & -1.05139 & -1.17130 & 1.30096 \\ \mathrm{H} & -0.66185 & -0.36918 & 1.69234 \\ \mathrm{H} & -0.61141 & -1.22577 & 0.41208 \\ \mathrm{O} & -2.07161 & 0.46755 & -1.57845 \\ \mathrm{H} & -1.32366 & -0.17295 & -1.51862 \\ \mathrm{H} & -2.72217 & 0.12157 & -0.94079 \\ \mathrm{O} & 0.26220 & 1.34320 & 1.92359 \\ \mathrm{H} & -0.02991 & 1.79254 & 1.07443 \\ \mathrm{H} & 0.08508 & 1.97500 & 2.62769 \\ \mathrm{O} & -0.40240 & 2.40944 & -0.42828 \\ \mathrm{H} & 0.39571 & 2.08209 & -0.90583 \\ \mathrm{H} & -1.11664 & 1.84748 & -0.80976 \\ \mathrm{O} & 1.71330 & 0.97859 & -1.54289 \\ \mathrm{H} & 1.12149 & 0.18915 & -1.49561 \\ \mathrm{H} & 2.27684 & 0.86644 & -0.75313\end{array}$

System F-(H2O)9 


$\begin{array}{llll}\mathrm{F} & 1.56141 & -0.38243 & -0.28984 \\ \mathrm{O} & -2.83574 & 0.30331 & 0.64066 \\ \mathrm{H} & -2.24947 & -0.28880 & 1.22059 \\ \mathrm{H} & -3.67798 & 0.36485 & 1.10296 \\ \mathrm{O} & -0.18566 & -2.40838 & -0.31694 \\ \mathrm{H} & -0.82602 & -2.14690 & -0.99480 \\ \mathrm{H} & 0.52534 & -1.71854 & -0.36402 \\ \mathrm{O} & 1.15814 & -0.19537 & 2.41453 \\ \mathrm{H} & 1.14116 & 0.77472 & 2.31311 \\ \mathrm{H} & 1.42466 & -0.45401 & 1.50318 \\ \mathrm{O} & -2.38690 & -0.93981 & -1.77905 \\ \mathrm{H} & -2.67246 & -0.48089 & -0.95383 \\ \mathrm{H} & -3.11242 & -0.84358 & -2.40420 \\ \mathrm{O} & 4.13028 & -0.72315 & -1.02307 \\ \mathrm{H} & 3.17513 & -0.65744 & -0.77979 \\ \mathrm{H} & 4.48838 & 0.12793 & -0.74871 \\ \mathrm{O} & 1.36092 & 2.23227 & 0.92675 \\ \mathrm{H} & 1.55522 & 1.44498 & 0.38262 \\ \mathrm{H} & 0.48663 & 2.51737 & 0.59395 \\ \mathrm{O} & -1.20045 & 2.61913 & -0.35748 \\ \mathrm{H} & -0.78888 & 2.08115 & -1.07292 \\ \mathrm{H} & -1.78566 & 1.98108 & 0.08381 \\ \mathrm{O} & -1.30733 & -1.32759 & 1.98441 \\ \mathrm{H} & -0.96642 & -1.86522 & 1.23023 \\ \mathrm{H} & -0.48607 & -0.87422 & 2.29809 \\ \mathrm{O} & -0.00618 & 0.80650 & -2.12688 \\ \mathrm{H} & 0.63998 & 0.36989 & -1.51849 \\ \mathrm{H} & -0.74037 & 0.17420 & -2.18667\end{array}$

\begin{tabular}{llll}
\multicolumn{5}{l}{ System F-(H2O) 10} & \\
F & 0.89974 & 1.69341 & -0.43895 \\
$\mathrm{O}$ & -2.23799 & -0.93876 & -1.61635 \\
$\mathrm{H}$ & -2.64326 & -0.22321 & -1.08786 \\
$\mathrm{H}$ & -1.36712 & -0.58142 & -1.90452 \\
$\mathrm{O}$ & 3.70417 & 1.24642 & -0.51797 \\
$\mathrm{H}$ & 2.77244 & 1.53435 & -0.55133 \\
$\mathrm{H}$ & 3.65456 & 0.41744 & -0.00855 \\
$\mathrm{O}$ & -3.12554 & 0.91556 & 0.34703 \\
$\mathrm{H}$ & -2.61072 & 0.44663 & 1.06190 \\
$\mathrm{H}$ & -4.00249 & 1.07195 & 0.71167 \\
$\mathrm{O}$ & 2.99936 & -1.13575 & 1.15496 \\
$\mathrm{H}$ & 2.31785 & -0.56467 & 1.55219 \\
$\mathrm{H}$ & 2.49223 & -1.68332 & 0.52169 \\
$\mathrm{O}$ & 0.36479 & -0.12366 & -2.08778 \\
$\mathrm{H}$ & 0.75584 & 0.14958 & -2.92404 \\
$\mathrm{H}$ & 0.58621 & 0.62653 & -1.43563 \\
$\mathrm{O}$ & 0.83167 & 0.72433 & 1.89405 \\
$\mathrm{H}$ & 1.04737 & 1.44808 & 2.49188
\end{tabular}




$\begin{array}{lrrr}\mathrm{H} & 0.85269 & 1.13830 & 0.96831 \\ \mathrm{O} & -1.46525 & -2.68320 & 0.25189 \\ \mathrm{H} & -1.97968 & -3.49570 & 0.20740 \\ \mathrm{H} & -1.80815 & -2.09760 & -0.48348 \\ \mathrm{O} & -1.35455 & 3.30120 & -0.36724 \\ \mathrm{H} & -1.99050 & 2.60274 & -0.14959 \\ \mathrm{H} & -0.50407 & 2.81848 & -0.43022 \\ \mathrm{O} & -1.64456 & -0.47103 & 2.10277 \\ \mathrm{H} & -0.75110 & -0.05247 & 2.09803 \\ \mathrm{H} & -1.52908 & -1.32172 & 1.64027 \\ \mathrm{O} & 1.31012 & -2.47160 & -0.74950 \\ \mathrm{H} & 1.08992 & -1.68828 & -1.29306 \\ \mathrm{H} & 0.46174 & -2.69463 & -0.32933\end{array}$

System F-(H2O) 11

$\begin{array}{llll}\mathrm{F} & 0.76327 & 0.96733 & 0.78089 \\ \mathrm{O} & -2.73308 & -1.89965 & 0.87481 \\ \mathrm{H} & -2.66918 & -2.38135 & 0.02559 \\ \mathrm{H} & -1.80332 & -1.84554 & 1.18151 \\ \mathrm{O} & 0.05321 & -1.54619 & -1.55763 \\ \mathrm{H} & -0.25608 & -0.61697 & -1.68474 \\ \mathrm{H} & 0.08302 & -1.62711 & -0.57906 \\ \mathrm{O} & -1.20504 & 2.63660 & 1.71340 \\ \mathrm{H} & -1.92718 & 2.04257 & 1.45027 \\ \mathrm{H} & -0.40236 & 2.10970 & 1.51008 \\ \mathrm{O} & 2.74631 & -2.18596 & 1.74406 \\ \mathrm{H} & 2.86418 & -1.54367 & 1.00396 \\ \mathrm{H} & 3.15982 & -1.75859 & 2.50270 \\ \mathrm{O} & -2.20572 & -3.08459 & -1.69073 \\ \mathrm{H} & -1.37263 & -2.55358 & -1.77391 \\ \mathrm{H} & -1.91498 & -4.00311 & -1.70419 \\ \mathrm{O} & 2.70946 & -0.30471 & -0.30336 \\ \mathrm{H} & 2.01240 & 0.28508 & 0.11837 \\ \mathrm{H} & 2.22919 & -0.73532 & -1.02744 \\ \mathrm{O} & -1.31127 & 4.13553 & -0.78526 \\ \mathrm{H} & -1.27044 & 3.79478 & 0.13037 \\ \mathrm{H} & -1.06977 & 3.35367 & -1.30467 \\ \mathrm{O} & 5.06146 & 1.08710 & -1.04594 \\ \mathrm{H} & 4.74763 & 1.98924 & -1.17360 \\ \mathrm{H} & 4.25296 & 0.59139 & -0.79809 \\ \mathrm{O} & -0.75951 & 1.11102 & -1.39996 \\ \mathrm{H} & -0.15449 & 1.17907 & -0.61914 \\ \mathrm{H} & -1.63885 & 1.03006 & -0.98467 \\ \mathrm{O} & -0.00253 & -1.46872 & 1.28660 \\ \mathrm{H} & 0.23123 & -0.50124 & 1.21867 \\ \mathrm{H} & 0.80757 & -1.88486 & 1.63462 \\ \mathrm{O} & -3.07159 & 0.72932 & 0.31399 \\ \mathrm{H} & -3.99085 & 0.93132 & 0.11203\end{array}$




\begin{tabular}{|c|c|c|c|}
\hline & 3.04090 & .23959 & 0 \\
\hline \multicolumn{4}{|c|}{ System F-(H2O)12 } \\
\hline 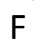 & -0.78491 & -0.91890 & -0.23315 \\
\hline & -4.37178 & -0.85306 & 1.33720 \\
\hline & -3.66089 & -0.17292 & 1.40735 \\
\hline & -4.02538 & -1.61318 & 1.81876 \\
\hline & 0.20723 & 2.68632 & 1.03923 \\
\hline & -0.55588 & 2.24731 & 1.44950 \\
\hline & 0.16252 & 2.34972 & 0.11783 \\
\hline 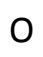 & 0.28932 & -1.58160 & 2.11417 \\
\hline & -0.07640 & -0.93089 & 2.72454 \\
\hline & -0.10798 & -1.34878 & 1.23837 \\
\hline 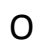 & 2.91682 & 826 & -1.65886 \\
\hline & 3.50510 & 1.28554 & -2.38604 \\
\hline & 3.07607 & 0.0 & -1.46730 \\
\hline 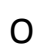 & 0.09049 & 1.3 & -1.44311 \\
\hline & 1.03284 & 194 & -1.69100 \\
\hline & -0.13827 & 0.45932 & -1.05825 \\
\hline b & 0.77469 & -2.73476 & -1.37912 \\
\hline & 0.14830 & -2.06937 & -0.99855 \\
\hline 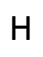 & 0.67520 & -3.50763 & -0.81199 \\
\hline כ & -2.80765 & 1.82093 & -1.46140 \\
\hline & -3.07264 & 0.89320 & -1.63959 \\
\hline$y$ & -1.87171 & 1.85285 & -1.72473 \\
\hline D & -3.29364 & -0.99891 & -1.43304 \\
\hline & -2.38154 & -1.12309 & -1.09760 \\
\hline 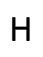 & -3.83501 & -1.06658 & -0.62613 \\
\hline ) & 3.06527 & -0.94184 & 1.73531 \\
\hline 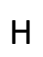 & 3.03255 & 0.03794 & 1.66755 \\
\hline $\mathrm{H}$ & 2.14177 & -1.19983 & 1.92934 \\
\hline 0 & -2.23008 & 0.92440 & 1.15618 \\
\hline $\mathrm{H}$ & -2.53596 & 1.43804 & 0.37440 \\
\hline $\mathrm{H}$ & -1.66807 & 0.24033 & 0.72546 \\
\hline 0 & 2.91164 & 1.82843 & 1.19091 \\
\hline $\mathrm{H}$ & 1.99077 & 2.14882 & 1.29472 \\
\hline $\mathrm{H}$ & 3.01622 & 1.73490 & 0.22755 \\
\hline O & 3.26147 & -1.47007 & -0.97491 \\
\hline $\mathrm{H}$ & 2.43506 & -1.96406 & -1.16538 \\
\hline & 3.26737 & -1.37457 & 0.00908 \\
\hline
\end{tabular}

System F-(H2O) 13

$\begin{array}{lrrr}\mathrm{F} & -1.43698 & -1.73786 & 0.94971 \\ \mathrm{O} & -2.26858 & 0.71604 & 1.62243 \\ \mathrm{H} & -1.99103 & -0.20755 & 1.39748 \\ \mathrm{H} & -3.17576 & 0.77715 & 1.26278 \\ \mathrm{O} & 0.58734 & 0.72001 & -1.21231 \\ \mathrm{H} & 0.26435 & -0.20011 & -1.41005\end{array}$




$\begin{array}{llll}\mathrm{H} & 0.57568 & 0.78454 & -0.23397 \\ \mathrm{O} & 2.02710 & -3.33645 & -1.53614 \\ \mathrm{H} & 2.57848 & -3.11959 & -2.29620 \\ \mathrm{H} & 2.46881 & -2.88655 & -0.76809 \\ \mathrm{O} & -1.86166 & 2.27789 & -0.82562 \\ \mathrm{H} & -1.18612 & 1.70924 & -1.23816 \\ \mathrm{H} & -1.92391 & 1.89579 & 0.07140 \\ \mathrm{O} & 3.08832 & -1.99272 & 0.57703 \\ \mathrm{H} & 2.33308 & -1.88997 & 1.18688 \\ \mathrm{H} & 3.29112 & -1.08316 & 0.26204 \\ \mathrm{O} & 0.74813 & -1.61815 & 2.21658 \\ \mathrm{H} & -0.13337 & -1.76087 & 1.74232 \\ \mathrm{H} & 0.65462 & -2.02740 & 3.08274 \\ \mathrm{O} & -0.35857 & -1.79604 & -1.50222 \\ \mathrm{H} & 0.40083 & -2.41351 & -1.55042 \\ \mathrm{H} & -0.71469 & -1.87507 & -0.58556 \\ \mathrm{O} & 3.44577 & 0.53184 & -0.59369 \\ \mathrm{H} & 2.58265 & 0.53388 & -1.04644 \\ \mathrm{H} & 3.39181 & 1.34420 & -0.04704 \\ \mathrm{O} & 2.65824 & 2.87230 & 0.86502 \\ \mathrm{H} & 1.96711 & 2.36972 & 1.33502 \\ \mathrm{H} & 2.15624 & 3.31625 & 0.15485 \\ \mathrm{O} & -3.54083 & -1.88858 & -0.69943 \\ \mathrm{H} & -3.03314 & -1.98267 & -1.51625 \\ \mathrm{H} & -2.84103 & -1.93308 & -0.00524 \\ \mathrm{O} & -4.45258 & 0.74918 & -0.23803 \\ \mathrm{H} & -4.22671 & -0.17549 & -0.48253 \\ \mathrm{H} & -3.84668 & 1.29054 & -0.76946 \\ \mathrm{O} & 0.54723 & 1.10289 & 1.65752 \\ \mathrm{H} & -0.40672 & 1.23683 & 1.80611 \\ \mathrm{H} & 0.71948 & 0.20123 & 2.00272 \\ \mathrm{O} & 0.89412 & 3.58901 & -1.35941 \\ \mathrm{H} & -0.02630 & 3.66800 & -1.06172 \\ \mathrm{H} & 0.94190 & 2.65075 & -1.61436\end{array}$

\begin{tabular}{|c|c|c|c|}
\hline \multicolumn{4}{|c|}{ System F-(H2O)14 } \\
\hline $\mathrm{F}$ & 0.15553 & 0.42251 & 0.32287 \\
\hline D & 3.83312 & -1.03382 & -1.52896 \\
\hline$H$ & 3.04729 & -1.64571 & -1.56263 \\
\hline 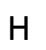 & 4.31162 & -1.17328 & -2.35243 \\
\hline ) & 0.20350 & 2.61208 & 2.07507 \\
\hline 4 & 0.28637 & 1.79316 & 1.54702 \\
\hline & -0.74184 & 2.63462 & 2.27645 \\
\hline 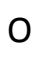 & -3.56662 & -1.65805 & 1.23922 \\
\hline $\mathrm{H}$ & -2.75844 & -2.23886 & 1.21524 \\
\hline & -4.21348 & -2.12377 & 1.77876 \\
\hline & -2.32559 & 0.93848 & 1.46157 \\
\hline & -1.46858 & 0.66184 & 1.07602 \\
\hline
\end{tabular}




$\begin{array}{llll}\mathrm{H} & -2.82728 & 0.10821 & 1.55125 \\ \mathrm{O} & -0.84624 & -1.50363 & -1.36967 \\ \mathrm{H} & -1.76083 & -1.24340 & -1.63616 \\ \mathrm{H} & -0.51049 & -0.74338 & -0.84800 \\ \mathrm{O} & 1.24233 & -1.84057 & 1.36152 \\ \mathrm{H} & 2.15757 & -1.60259 & 1.61462 \\ \mathrm{H} & 0.84062 & -0.98551 & 1.08346 \\ \mathrm{O} & -3.21552 & 1.96565 & -1.06957 \\ \mathrm{H} & -2.34213 & 2.26972 & -1.39717 \\ \mathrm{H} & -3.03106 & 1.76813 & -0.12944 \\ \mathrm{O} & -3.48726 & -0.74708 & -1.64830 \\ \mathrm{H} & -3.45514 & 0.23569 & -1.55544 \\ \mathrm{H} & -3.74448 & -1.06012 & -0.76511 \\ \mathrm{O} & 3.91555 & -0.80900 & 1.47639 \\ \mathrm{H} & 3.57497 & 0.06584 & 1.20019 \\ \mathrm{H} & 4.22781 & -1.16379 & 0.62862 \\ \mathrm{O} & 2.70212 & 1.24240 & -0.12828 \\ \mathrm{H} & 1.77569 & 0.92477 & -0.03288 \\ \mathrm{H} & 3.08075 & 0.67810 & -0.82606 \\ \mathrm{O} & -0.49265 & 2.39765 & -1.65366 \\ \mathrm{H} & 0.07900 & 3.10750 & -1.29928 \\ \mathrm{H} & -0.26227 & 1.64326 & -1.08182 \\ \mathrm{O} & 1.71922 & -2.66688 & -1.31133 \\ \mathrm{H} & 1.58085 & -2.54733 & -0.34594 \\ \mathrm{H} & 0.86742 & -2.36329 & -1.67789 \\ \mathrm{O} & 1.45386 & 3.92584 & -0.16707 \\ \mathrm{H} & 2.09566 & 3.19881 & -0.25772 \\ \mathrm{H} & 1.00933 & 3.71486 & 0.67713 \\ \mathrm{O} & -1.32891 & -3.09295 & 0.86929 \\ \mathrm{H} & -1.15317 & -2.67271 & -0.00498 \\ \mathrm{H} & -0.52082 & -2.88427 & 1.36873\end{array}$

\begin{tabular}{lccl}
\multicolumn{4}{l}{ System F-(H2O)15 } \\
F & 0.31160 & 0.76384 & 0.69550 \\
$\mathrm{O}$ & 0.77822 & -3.13317 & -1.06158 \\
$\mathrm{H}$ & 0.59232 & -2.81060 & -0.15561 \\
$\mathrm{H}$ & -0.07149 & -3.01247 & -1.51549 \\
$\mathrm{O}$ & 2.31523 & 1.22927 & -1.20359 \\
$\mathrm{H}$ & 1.67746 & 1.05026 & -0.48651 \\
$\mathrm{H}$ & 2.51130 & 0.32481 & -1.55017 \\
$\mathrm{O}$ & -1.12616 & 0.32233 & -1.67813 \\
$\mathrm{H}$ & -1.20508 & -0.62241 & -1.90096 \\
$\mathrm{H}$ & -0.50324 & 0.37856 & -0.92738 \\
$\mathrm{O}$ & -3.91717 & -1.68665 & -0.32420 \\
$\mathrm{H}$ & -3.99528 & -0.71768 & -0.40287 \\
$\mathrm{H}$ & -3.57295 & -1.82462 & 0.58063 \\
$\mathrm{O}$ & -2.71069 & 3.91174 & -0.54689 \\
$\mathrm{H}$ & -1.74544 & 3.70234 & -0.49197
\end{tabular}




$\begin{array}{rrrr}\mathrm{H} & -2.89688 & 4.38145 & 0.27393 \\ \mathrm{O} & 2.99569 & -1.39877 & -1.72641 \\ \mathrm{H} & 2.26439 & -2.02685 & -1.55892 \\ \mathrm{H} & 3.55879 & -1.42140 & -0.92441 \\ \mathrm{O} & 4.55050 & -0.91567 & 0.64051 \\ \mathrm{H} & 3.84896 & -0.75237 & 1.30232 \\ \mathrm{H} & 4.81940 & -0.01374 & 0.38094 \\ \mathrm{O} & 0.15298 & -1.88342 & 1.38569 \\ \mathrm{H} & -0.74695 & -2.03170 & 1.72659 \\ \mathrm{H} & 0.15241 & -0.95064 & 1.07587 \\ \mathrm{O} & -3.40574 & 1.16120 & -0.50908 \\ \mathrm{H} & -3.41155 & 2.13644 & -0.58793 \\ \mathrm{H} & -2.59894 & 0.88729 & -1.01902 \\ \mathrm{O} & -1.94174 & -2.40645 & -2.06804 \\ \mathrm{H} & -2.68932 & -2.21747 & -1.44380 \\ \mathrm{H} & -2.35276 & -2.64582 & -2.90527 \\ \mathrm{O} & -2.62450 & -1.74128 & 2.22285 \\ \mathrm{H} & -2.45650 & -0.74959 & 2.23018 \\ \mathrm{H} & -2.97047 & -1.96615 & 3.09282 \\ \mathrm{O} & -2.11101 & 0.82417 & 1.94908 \\ \mathrm{H} & -1.18502 & 0.87433 & 1.62125 \\ \mathrm{H} & -2.64316 & 1.01897 & 1.14425 \\ \mathrm{O} & 2.35580 & -0.28452 & 2.42237 \\ \mathrm{H} & 1.78478 & 0.31068 & 1.90142 \\ \mathrm{H} & 1.82065 & -1.09465 & 2.44039 \\ \mathrm{O} & -0.04752 & 3.27204 & -0.25669 \\ \mathrm{H} & 0.48785 & 3.16457 & -1.05478 \\ \mathrm{H} & 0.05998 & 2.40577 & 0.19886 \\ \mathrm{O} & 4.76105 & 1.87830 & -0.16567 \\ \mathrm{H} & 4.60030 & 2.42163 & 0.61406 \\ \mathrm{H} & 3.86259 & 1.73348 & -0.55976\end{array}$

\begin{tabular}{lccc}
\multicolumn{5}{l}{ System F-(H2O)16 } \\
F & 0.51073 & -1.38031 & -1.87002 \\
O & -0.05711 & -3.05136 & 0.10967 \\
H & 0.12447 & -2.40118 & -0.61765 \\
H & -1.02757 & -3.12645 & 0.12137 \\
O & -2.11686 & -0.54704 & 2.41972 \\
H & -2.84018 & -0.63848 & 3.04948 \\
H & -2.17543 & 0.38978 & 2.08712 \\
O & -0.57241 & 2.85356 & -1.68933 \\
H & -0.26773 & 3.11108 & -0.80045 \\
H & 0.11373 & 2.20550 & -2.01365 \\
O & -5.19152 & -0.42582 & 0.03445 \\
H & -4.60004 & 0.24165 & -0.33785 \\
H & -4.61695 & -1.21319 & 0.09413 \\
O & 3.73317 & 1.42522 & -0.91885 \\
H & 4.53766 & 1.75643 & -1.33104
\end{tabular}




$\begin{array}{lrrr}\mathrm{H} & 3.93974 & 0.48879 & -0.61769 \\ \mathrm{O} & 2.70939 & -2.72649 & -1.52738 \\ \mathrm{H} & 2.33651 & -3.46364 & -1.02734 \\ \mathrm{H} & 1.90136 & -2.19516 & -1.77331 \\ \mathrm{O} & -2.94824 & -2.30703 & 0.20057 \\ \mathrm{H} & -2.62205 & -1.71527 & 0.90345 \\ \mathrm{H} & -2.66071 & -1.88121 & -0.64821 \\ \mathrm{O} & 0.42339 & -1.81049 & 2.47954 \\ \mathrm{H} & -0.41443 & -1.31935 & 2.58028 \\ \mathrm{H} & 0.30581 & -2.30170 & 1.62477 \\ \mathrm{O} & 2.63113 & -0.41900 & 2.44141 \\ \mathrm{H} & 1.76006 & -0.92959 & 2.45532 \\ \mathrm{H} & 3.05769 & -0.62295 & 3.28045 \\ \mathrm{O} & -2.89948 & 1.69664 & -1.32223 \\ \mathrm{H} & -2.01467 & 2.14078 & -1.51741 \\ \mathrm{H} & -3.54472 & 2.17507 & -1.85485 \\ \mathrm{O} & 1.26910 & 1.07492 & -2.48017 \\ \mathrm{H} & 2.11618 & 1.20150 & -2.01978 \\ \mathrm{H} & 0.99366 & 0.14589 & -2.26568 \\ \mathrm{O} & 0.13649 & 3.32974 & 1.08054 \\ \mathrm{H} & 1.01643 & 2.88335 & 1.25163 \\ \mathrm{H} & 0.22264 & 4.21443 & 1.45086 \\ \mathrm{O} & -2.30903 & 1.95472 & 1.47961 \\ \mathrm{H} & -1.44938 & 2.41596 & 1.40785 \\ \mathrm{H} & -2.64043 & 1.90019 & 0.56228 \\ \mathrm{O} & 4.12422 & -0.99933 & 0.04047 \\ \mathrm{H} & 3.63049 & -0.93675 & 0.88042 \\ \mathrm{H} & 3.63687 & -1.66666 & -0.50693 \\ \mathrm{O} & 2.50852 & 2.22281 & 1.48899 \\ \mathrm{H} & 2.50423 & 1.33582 & 1.90689 \\ \mathrm{H} & 2.96887 & 2.07882 & 0.63628 \\ \mathrm{O} & -2.12002 & -1.08899 & -2.09444 \\ \mathrm{H} & -1.13309 & -1.15905 & -2.10702 \\ \mathrm{H} & -2.32150 & -0.14821 & -1.96405\end{array}$

\begin{tabular}{lccc}
\multicolumn{5}{l}{ System F-(H2O) 17} & \\
$\mathrm{~F}$ & 0.14016 & 1.35708 & 1.76386 \\
$\mathrm{O}$ & 4.12203 & -0.17935 & 0.49207 \\
$\mathrm{H}$ & 4.95343 & -0.65890 & 0.57132 \\
$\mathrm{H}$ & 3.68098 & -0.54275 & -0.32256 \\
$\mathrm{O}$ & -3.55404 & -2.17510 & -0.40319 \\
$\mathrm{H}$ & -3.68310 & -1.25336 & -0.69803 \\
$\mathrm{H}$ & -2.80707 & -2.47117 & -0.95578 \\
$\mathrm{O}$ & 2.84115 & -1.14995 & -1.66868 \\
$\mathrm{H}$ & 2.30771 & -1.92640 & -1.40878 \\
$\mathrm{H}$ & 2.21212 & -0.48245 & -2.00868 \\
$\mathrm{O}$ & 1.13574 & 0.95209 & -2.63699 \\
$\mathrm{H}$ & 0.23042 & 0.67497 & -2.94868
\end{tabular}




$\begin{array}{rrrr}\mathrm{H} & 1.54777 & 1.38576 & -3.39270 \\ \mathrm{O} & 4.26764 & 2.74639 & -0.18646 \\ \mathrm{H} & 4.33779 & 1.81462 & 0.07800 \\ \mathrm{H} & 3.30366 & 2.88663 & -0.22826 \\ \mathrm{O} & 1.38148 & -3.44388 & -0.76454 \\ \mathrm{H} & 1.16996 & -3.27553 & 0.20514 \\ \mathrm{H} & 1.83006 & -4.29532 & -0.79087 \\ \mathrm{O} & -0.96459 & -2.27956 & -1.85579 \\ \mathrm{H} & -0.75590 & -1.55301 & -1.22827 \\ \mathrm{H} & -0.23738 & -2.90916 & -1.69947 \\ \mathrm{O} & -1.27735 & 0.05662 & -3.45953 \\ \mathrm{H} & -1.18415 & -0.88017 & -3.20086 \\ \mathrm{H} & -1.98870 & 0.37730 & -2.86334 \\ \mathrm{O} & -1.17637 & 3.58159 & 0.87272 \\ \mathrm{H} & -0.46293 & 3.71446 & 0.22553 \\ \mathrm{H} & -0.83669 & 2.80327 & 1.36571 \\ \mathrm{O} & 2.00520 & -0.54569 & 2.41327 \\ \mathrm{H} & 1.39622 & 0.20087 & 2.22599 \\ \mathrm{H} & 2.77696 & -0.39156 & 1.83553 \\ \mathrm{O} & -3.05001 & 0.56417 & -1.31137 \\ \mathrm{H} & -3.35866 & 1.44555 & -1.00932 \\ \mathrm{H} & -2.27159 & 0.37058 & -0.73918 \\ \mathrm{O} & 0.80978 & -2.93900 & 1.76262 \\ \mathrm{H} & 1.24330 & -2.08807 & 2.02626 \\ \mathrm{H} & -0.14652 & -2.78164 & 1.87509 \\ \mathrm{O} & 1.34360 & 2.71786 & -0.34040 \\ \mathrm{H} & 1.08120 & 2.18968 & 0.44109 \\ \mathrm{H} & 1.22158 & 2.12016 & -1.10258 \\ \mathrm{O} & -3.61118 & 3.09439 & -0.23334 \\ \mathrm{H} & -4.21407 & 3.07917 & 0.51824 \\ \mathrm{H} & -2.72439 & 3.30178 & 0.16154 \\ \mathrm{O} & -1.62675 & 0.24297 & 3.41556 \\ \mathrm{H} & -1.15366 & 0.08245 & 4.23973 \\ \mathrm{H} & -0.96842 & 0.71313 & 2.84201 \\ \mathrm{O} & -0.86069 & -0.33252 & 0.06856 \\ \mathrm{H} & -0.38778 & 0.34800 & 0.61442 \\ \mathrm{H} & -1.17431 & -0.97745 & 0.73439 \\ \mathrm{H} & -1.96279 & -2.12686 & 1.95030 \\ \mathrm{H} & -2.07617 & -1.34612 & 2.53288 \\ & & -2.16247 & 1.31138\end{array}$

System F-(H2O) 18

$\begin{array}{lrrr}\text { F } & -0.57710 & 0.26010 & -0.15109 \\ \mathrm{O} & 0.01539 & -3.38463 & 0.80523 \\ \mathrm{H} & -0.74143 & -3.13008 & 0.23423 \\ \mathrm{H} & 0.80588 & -3.19978 & 0.26870 \\ \mathrm{O} & 0.14689 & 2.70261 & -2.50067 \\ \mathrm{H} & -0.75406 & 2.32691 & -2.48901\end{array}$




\begin{tabular}{|c|c|c|c|}
\hline $\mathrm{H}$ & 0.73295 & 1.92659 & -2.48484 \\
\hline $\mathrm{O}$ & 2.63423 & -0.82153 & 2.11518 \\
\hline $\mathrm{H}$ & 3.00393 & -0.99549 & 2.98708 \\
\hline $\mathrm{H}$ & 1.64080 & -0.78827 & 2.23479 \\
\hline O & 3.63813 & 1.15437 & 0.32254 \\
\hline $\mathrm{H}$ & 4.50393 & 0.82217 & 0.00619 \\
\hline $\mathrm{H}$ & 3.38036 & 0.55690 & 1.05046 \\
\hline $\mathrm{O}$ & -2.37791 & 1.46419 & -1.70390 \\
\hline $\mathrm{H}$ & -2.83901 & 2.02992 & -1.06304 \\
\hline $\mathrm{H}$ & -1.74983 & 0.96308 & -1.12879 \\
\hline 0 & 2.54602 & -2.24469 & -0.37992 \\
\hline $\mathrm{H}$ & 2.21783 & -1.47625 & -0.90921 \\
\hline $\mathrm{H}$ & 2.66959 & -1.86678 & 0.51345 \\
\hline $\mathrm{O}$ & -2.69275 & 2.80484 & 0.94258 \\
\hline $\mathrm{H}$ & -3.05954 & 3.57663 & 1.38696 \\
\hline $\mathrm{H}$ & -1.73773 & 3.01059 & 0.77724 \\
\hline $\mathrm{O}$ & -2.07548 & -2.07516 & -0.56049 \\
\hline $\mathrm{H}$ & -2.78933 & -2.00354 & 0.12525 \\
\hline $\mathrm{H}$ & -1.55762 & -1.25124 & -0.45506 \\
\hline $\mathrm{O}$ & -5.97940 & -0.89610 & -0.74054 \\
\hline $\mathrm{H}$ & -6.55050 & -1.62202 & -1.01467 \\
\hline $\mathrm{H}$ & -5.26265 & -0.86217 & -1.42582 \\
\hline 0 & 2.60546 & 3.69725 & 1.41912 \\
\hline $\mathrm{H}$ & 1.69061 & 3.50969 & 1.14874 \\
\hline $\mathrm{H}$ & 3.10393 & 2.95358 & 1.04110 \\
\hline $\mathrm{O}$ & 6.01628 & 0.01645 & -0.73521 \\
\hline $\mathrm{H}$ & 6.18429 & 0.40512 & -1.60132 \\
\hline $\mathrm{H}$ & 5.75518 & -0.91526 & -0.92312 \\
\hline $\mathrm{O}$ & -2.76326 & 0.35816 & 2.65422 \\
\hline $\mathrm{H}$ & -2.70132 & 1.15183 & 2.09140 \\
\hline $\mathrm{H}$ & -1.86893 & -0.02525 & 2.65919 \\
\hline $\mathrm{O}$ & 5.10494 & -2.53383 & -1.24790 \\
\hline $\mathrm{H}$ & 4.15491 & -2.50800 & -0.96239 \\
\hline $\mathrm{H}$ & 5.48059 & -3.31357 & -0.82553 \\
\hline $\mathrm{O}$ & -3.85795 & -0.85691 & -2.45433 \\
\hline $\mathrm{H}$ & -3.23630 & -1.43639 & -1.96729 \\
\hline $\mathrm{H}$ & -3.44551 & 0.02474 & -2.35120 \\
\hline $\mathrm{O}$ & -4.09390 & -1.70750 & 1.25451 \\
\hline $\mathrm{H}$ & -4.82711 & -1.36555 & 0.70600 \\
\hline $\mathrm{H}$ & -3.76508 & -0.95147 & 1.78578 \\
\hline $\mathrm{O}$ & -0.05183 & 2.88970 & 0.32498 \\
\hline $\mathrm{H}$ & 0.08689 & 3.08784 & -0.62657 \\
\hline $\mathrm{H}$ & -0.11781 & 1.90977 & 0.30980 \\
\hline 0 & 1.70242 & 0.16210 & -1.51816 \\
\hline $\mathrm{H}$ & 2.34492 & 0.64357 & -0.95400 \\
\hline $\mathrm{H}$ & 0.85055 & 0.18595 & -1.02001 \\
\hline $\mathrm{O}$ & -0.02833 & -0.90891 & 2.13779 \\
\hline $\mathrm{H}$ & -0.09650 & -1.85186 & 1.85981 \\
\hline
\end{tabular}




\begin{tabular}{|c|c|c|c|}
\hline & -0.22454 & -0.42611 & 1.295 \\
\hline \multicolumn{4}{|c|}{ System F-(H2O)19 } \\
\hline 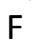 & -2.09543 & 0.84773 & 1.24615 \\
\hline & 0.27505 & 0.91717 & -2.11816 \\
\hline y & -0.64650 & 1.24942 & -2.28679 \\
\hline & 0.27846 & 0.66407 & -1.15671 \\
\hline P & -2.10531 & 3.15468 & 0.16526 \\
\hline y & -2.70415 & 3.74616 & 0.63188 \\
\hline & -2.12631 & 2.28068 & 0.66680 \\
\hline 9 & 0.46191 & 3.73818 & -0.71809 \\
\hline H & 0.60511 & 2.96118 & -1.28198 \\
\hline 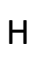 & -0.44774 & 3.61484 & -0.36643 \\
\hline 0 & 2.31122 & 1.27822 & 1.99326 \\
\hline & 2.30877 & 2.25548 & 1.89363 \\
\hline & 3.03588 & 0.96661 & 1.41415 \\
\hline 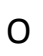 & 3.54281 & -2.54271 & 0893 \\
\hline y & 3.88179 & -1.63957 & 0.12452 \\
\hline H & 2.73046 & -2.56611 & 0.53862 \\
\hline 0 & 4.16694 & 0.32825 & 0.06409 \\
\hline$y$ & 5.07675 & 0.64021 & 0.01311 \\
\hline $\mathrm{H}$ & 3.78052 & 0.47316 & -0.84858 \\
\hline $\mathrm{O}$ & -0.01387 & -1.72699 & -2.87796 \\
\hline y & 0.07884 & -0.76110 & -2.67894 \\
\hline 1 & -0.39590 & -1.76727 & -3.76248 \\
\hline 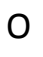 & 1.1 & -0.80997 & 3.76462 \\
\hline-1 & 0.16519 & -0.6 & 3.71208 \\
\hline H & 1.52136 & 439 & 3.39039 \\
\hline 0 & 0.27800 & 0.18749 & 0.46234 \\
\hline | & 0.92311 & 0.68134 & 1.01750 \\
\hline $\mathrm{H}$ & -0.62990 & 0.42805 & 0.80214 \\
\hline 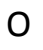 & -3.49576 & -0.24282 & -0.76355 \\
\hline H & -3.03988 & 0.10661 & 0.03954 \\
\hline H & -3.09846 & -1.13137 & -0.91184 \\
\hline 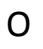 & 3.14437 & 0.51772 & -2.39015 \\
\hline $\mathrm{H}$ & 3.08923 & -0.44486 & -2.61446 \\
\hline $\mathrm{H}$ & 2.22010 & 0.82252 & -2.42739 \\
\hline 0 & 1.12196 & -2.25407 & 1.43632 \\
\hline $\mathrm{H}$ & 0.80 & -1.45443 & 0.95413 \\
\hline $\mathrm{H}$ & 1.25804 & -1.88399 & 2.34506 \\
\hline $\mathrm{O}$ & -2.17904 & -2.66145 & -1.13859 \\
\hline $\mathrm{H}$ & -1.37493 & -2.43234 & -1.63939 \\
\hline $\mathrm{H}$ & -1.87876 & -2.94731 & -0.25423 \\
\hline ? & 2.85342 & -2.20281 & -2.70112 \\
\hline $\mathrm{H}$ & 3.07105 & -2.45881 & -1.77574 \\
\hline $\mathrm{H}$ & 1.88200 & -2.24676 & -2.75891 \\
\hline $\mathrm{O}$ & 2.13549 & 3.98635 & 1.39817 \\
\hline 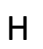 & 2.90487 & 4.48394 & 1.10167 \\
\hline
\end{tabular}




$\begin{array}{llll}\mathrm{H} & 1.54252 & 3.92373 & 0.60553 \\ \mathrm{O} & -1.64542 & -0.83784 & 3.31833 \\ \mathrm{H} & -1.89432 & -0.17117 & 2.63852 \\ \mathrm{H} & -1.74832 & -1.68705 & 2.85936 \\ \mathrm{O} & -6.29719 & -0.21843 & -0.42897 \\ \mathrm{H} & -5.32664 & -0.22877 & -0.57824 \\ \mathrm{H} & -6.39493 & -0.56253 & 0.46554 \\ \mathrm{O} & -1.33983 & -3.33111 & 1.53656 \\ \mathrm{H} & -1.35409 & -4.25829 & 1.79749 \\ \mathrm{H} & -0.38252 & -3.06374 & 1.52468 \\ \mathrm{O} & -2.31453 & 1.72931 & -2.34507 \\ \mathrm{H} & -2.38298 & 2.41887 & -1.66027 \\ \mathrm{H} & -2.80844 & 0.98076 & -1.93617\end{array}$

System F-(H2O)20

$\begin{array}{llll}\mathrm{O} & -2.92995 & -3.69563 & -1.22765 \\ \mathrm{H} & -1.96784 & -3.56875 & -1.24806 \\ \mathrm{H} & -3.28024 & -2.88563 & -0.82354 \\ \mathrm{O} & -0.00437 & -2.88580 & -1.16235 \\ \mathrm{H} & 0.00044 & -2.54455 & -0.23738 \\ \mathrm{H} & 0.93751 & -3.04268 & -1.35327 \\ \mathrm{O} & 1.05373 & -0.01599 & 3.23463 \\ \mathrm{H} & 2.03390 & -0.05031 & 3.16960 \\ \mathrm{H} & 0.76801 & -0.67609 & 2.56573 \\ \mathrm{O} & -4.28157 & -1.14167 & -0.23250 \\ \mathrm{H} & -4.12494 & -0.73436 & 0.67314 \\ \mathrm{H} & -5.22937 & -1.29814 & -0.29440 \\ \mathrm{O} & -2.64024 & 0.35425 & -1.81777 \\ \mathrm{H} & -2.75343 & 1.31378 & -1.68768 \\ \mathrm{H} & -3.34617 & -0.11321 & -1.31829 \\ \mathrm{O} & -2.74756 & 2.63480 & 1.63263 \\ \mathrm{H} & -2.69394 & 2.83863 & 0.68224 \\ \mathrm{H} & -1.81033 & 2.54314 & 1.89495 \\ \mathrm{O} & -0.27858 & 0.38784 & -0.12699 \\ \mathrm{H} & -1.09892 & 0.28616 & -0.64330 \\ \mathrm{H} & -0.13402 & -0.46631 & 0.34789 \\ \mathrm{O} & 3.37850 & -0.17299 & -2.04208 \\ \mathrm{H} & 3.52957 & 0.37173 & -1.24660 \\ \mathrm{H} & 2.59680 & 0.22748 & -2.47859 \\ \mathrm{O} & -2.33671 & 3.11383 & -1.27260 \\ \mathrm{H} & -2.75625 & 3.85261 & -1.72591 \\ \mathrm{H} & -1.36836 & 3.17398 & -1.50738 \\ \mathrm{O} & 1.85485 & 3.39290 & 0.48877 \\ \mathrm{H} & 1.18053 & 2.93661 & 1.09361 \\ \mathrm{H} & 1.99957 & 4.26625 & 0.86877 \\ \mathrm{O} & 2.74506 & -2.66961 & 1.42693 \\ \mathrm{H} & 1.78802 & -2.44442 & 1.44350 \\ \mathrm{H} & 2.93124 & -2.79669 & 0.48039\end{array}$




$\begin{array}{llll}\mathrm{O} & -1.74550 & -1.51511 & 3.17236 \\ \mathrm{H} & -1.12207 & -1.75092 & 2.44417 \\ \mathrm{H} & -1.17848 & -1.05646 & 3.80611 \\ \mathrm{O} & 1.11208 & 0.90302 & -3.31558 \\ \mathrm{H} & 1.23787 & 1.03039 & -4.26251 \\ \mathrm{H} & 0.44062 & 0.15058 & -3.23896 \\ \mathrm{O} & -3.78719 & 0.00748 & 2.08567 \\ \mathrm{H} & -3.44509 & 0.91699 & 1.95295 \\ \mathrm{H} & -3.06122 & -0.49655 & 2.52181 \\ \mathrm{O} & 0.05423 & 2.09601 & 1.84003 \\ \mathrm{H} & 0.42929 & 1.45375 & 2.49602 \\ \mathrm{H} & -0.15889 & 1.48869 & 1.07098 \\ \mathrm{O} & -0.65185 & -1.02704 & -3.17805 \\ \mathrm{H} & -0.43156 & -1.69260 & -2.48670 \\ \mathrm{H} & -1.45563 & -0.58780 & -2.83331 \\ \mathrm{O} & 3.88808 & 1.53616 & 0.23417 \\ \mathrm{H} & 4.72057 & 2.01005 & 0.13131 \\ \mathrm{H} & 3.19702 & 2.23322 & 0.33200 \\ \mathrm{O} & 0.26408 & 3.24315 & -1.94561 \\ \mathrm{H} & 0.54326 & 2.41683 & -2.39490 \\ \mathrm{H} & 0.83655 & 3.30914 & -1.15823 \\ \mathrm{O} & 3.76288 & -0.34522 & 2.54994 \\ \mathrm{H} & 3.52227 & -1.21057 & 2.14125 \\ \mathrm{H} & 3.84710 & 0.26170 & 1.79578 \\ \mathrm{O} & 2.89251 & -2.76029 & -1.47430 \\ \mathrm{H} & 3.07546 & -1.80670 & -1.69454 \\ \mathrm{H} & 3.49674 & -3.27487 & -2.01921 \\ \mathrm{~F} & 0.16494 & -1.78940 & 1.30927\end{array}$

(f) $\mathrm{Li}^{+}\left(\mathrm{H}_{2} \mathrm{O}\right)_{\mathrm{n}}(\mathrm{n}=1-20)$ systems, M06-2X/6-311+G(d,p) System $\mathrm{Li}+(\mathrm{H} 2 \mathrm{O}) 1$

Li $\quad-0.00000 \quad-0.00000 \quad-1.49623$

$\begin{array}{llll}\text { O } & 0.00000 & 0.00000 & 0.33405\end{array}$

$\begin{array}{llll}\mathrm{H} & 0.00000 & 0.77567 & 0.90814\end{array}$

$\begin{array}{llll}H & -0.00000 & -0.77567 & 0.90814\end{array}$

System $\mathrm{Li}+(\mathrm{H} 2 \mathrm{O}) 2$

Li $\quad 0.00000 \quad-0.00022 \quad-0.00026$

$\begin{array}{llll}\mathrm{O} & -1.85940 & 0.00002 & 0.00002\end{array}$

$\begin{array}{llll}\mathrm{H} & -2.43223 & 0.54663 & -0.54970\end{array}$

$\begin{array}{llll}\text { H } & -2.43220 & -0.54647 & 0.54990\end{array}$

$\begin{array}{llll}O & 1.85940 & 0.00002 & 0.00002\end{array}$

$\begin{array}{llll}\mathrm{H} & 2.43204 & 0.54992 & 0.54665\end{array}$

$\begin{array}{llll}H & 2.43238 & -0.54971 & -0.54642\end{array}$

System $\mathrm{Li}+(\mathrm{H} 2 \mathrm{O}) 3$

Li $\quad-0.00143 \quad-0.00140 \quad-0.00036$

$\begin{array}{llll}0 & -1.82754 & -0.45000 & 0.00004\end{array}$ 


$\begin{array}{rrrr}\mathrm{H} & -2.23541 & -1.18062 & 0.47618 \\ \mathrm{H} & -2.52727 & 0.00866 & -0.47628 \\ \mathrm{O} & 1.30460 & -1.35425 & -0.00010 \\ \mathrm{H} & 1.25469 & -2.19458 & -0.46734 \\ \mathrm{H} & 2.14593 & -1.33522 & 0.46758 \\ \mathrm{O} & 0.52319 & 1.80447 & 0.00011 \\ \mathrm{H} & 1.27696 & 2.17958 & -0.46674 \\ \mathrm{H} & 0.08737 & 2.52463 & 0.46731\end{array}$

\begin{tabular}{lccc}
\multicolumn{5}{l}{ System $\mathrm{Li}+(\mathrm{H} 2 \mathrm{O}) 4$} & \\
$\mathrm{Li}$ & -0.00210 & -0.19039 & -0.06861 \\
$\mathrm{O}$ & 1.70117 & -1.03543 & -0.22136 \\
$\mathrm{H}$ & 2.36134 & -0.93415 & 0.47106 \\
$\mathrm{H}$ & 2.02702 & -1.69776 & -0.83787 \\
$\mathrm{O}$ & -1.71162 & -1.02327 & -0.21539 \\
$\mathrm{H}$ & -2.04416 & -1.68058 & -0.83372 \\
$\mathrm{H}$ & -2.36904 & -0.92014 & 0.47935 \\
$\mathrm{O}$ & 0.00739 & 0.70917 & 1.61988 \\
$\mathrm{H}$ & 0.00693 & 0.46002 & 2.54849 \\
$\mathrm{H}$ & 0.01145 & 1.67037 & 1.56914 \\
$\mathrm{O}$ & 0.00353 & 1.37726 & -1.17597 \\
$\mathrm{H}$ & 0.78130 & 1.72080 & -1.62621 \\
$\mathrm{H}$ & -0.77234 & 1.73082 & -1.62170
\end{tabular}

System $\mathrm{Li}+(\mathrm{H} 2 \mathrm{O}) 5$

$\begin{array}{llll}\mathrm{Li} & 0.82294 & -0.00000 & 0.00000 \\ \mathrm{O} & 1.87712 & 1.41755 & 0.69952 \\ \mathrm{H} & 2.70662 & 1.44928 & 1.18363 \\ \mathrm{H} & 1.51580 & 2.30816 & 0.66584 \\ \mathrm{O} & -0.46495 & 1.03122 & -0.98401 \\ \mathrm{H} & -0.38119 & 1.45248 & -1.84325 \\ \mathrm{H} & -1.40187 & 0.83872 & -0.82315 \\ \mathrm{O} & -2.87975 & -0.00000 & -0.00000 \\ \mathrm{H} & -3.45215 & -0.55632 & -0.53999 \\ \mathrm{H} & -3.45215 & 0.55632 & 0.53999 \\ \mathrm{O} & -0.46495 & -1.03122 & 0.98401 \\ \mathrm{H} & -0.38119 & -1.45248 & 1.84325 \\ \mathrm{H} & -1.40187 & -0.83872 & 0.82315 \\ \mathrm{O} & 1.87712 & -1.41755 & -0.69952 \\ \mathrm{H} & 2.70662 & -1.44928 & -1.18363 \\ \mathrm{H} & 1.51580 & -2.30816 & -0.66585\end{array}$

\begin{tabular}{lccc}
\multicolumn{5}{l}{ System $\mathrm{Li}+(\mathrm{H} 2 \mathrm{O}) 6$} & & \\
$\mathrm{Li}$ & -0.00000 & 0.00001 & 0.00000 \\
$\mathrm{O}$ & 1.31250 & 0.98240 & -0.98342 \\
$\mathrm{H}$ & 2.25602 & 0.82798 & -0.82873 \\
$\mathrm{H}$ & 1.20896 & 1.65656 & -1.65847 \\
$\mathrm{O}$ & -1.31249 & -0.98345 & -0.98236
\end{tabular}




$\begin{array}{lrrr}\mathrm{H} & -1.20894 & -1.65853 & -1.65651 \\ \mathrm{H} & -2.25601 & -0.82875 & -0.82798 \\ \mathrm{O} & -3.74859 & -0.00001 & -0.00000 \\ \mathrm{H} & -4.31996 & -0.54816 & 0.54875 \\ \mathrm{H} & -4.31998 & 0.54813 & -0.54874 \\ \mathrm{O} & 1.31249 & -0.98238 & 0.98344 \\ \mathrm{H} & 2.25601 & -0.82797 & 0.82875 \\ \mathrm{H} & 1.20895 & -1.65653 & 1.65850 \\ \mathrm{O} & -1.31250 & 0.98346 & 0.98236 \\ \mathrm{H} & -2.25602 & 0.82876 & 0.82796 \\ \mathrm{H} & -1.20896 & 1.65855 & 1.65649 \\ \mathrm{O} & 3.74859 & -0.00001 & -0.00001 \\ \mathrm{H} & 4.31995 & -0.54876 & -0.54816 \\ \mathrm{H} & 4.31998 & 0.54872 & 0.54814\end{array}$

System Li+(H2O)7

$\begin{array}{cccc}\mathrm{Li} & 1.52757 & 0.29152 & -0.30195 \\ \mathrm{O} & 3.36098 & -0.16428 & -0.07745 \\ \mathrm{H} & 3.49352 & -0.63633 & 0.75056 \\ \mathrm{H} & 4.21415 & -0.03933 & -0.50027 \\ \mathrm{O} & -1.99073 & 1.38601 & 0.50265 \\ \mathrm{H} & -2.60640 & 2.03688 & 0.85351 \\ \mathrm{H} & -1.94883 & 0.63076 & 1.11644 \\ \mathrm{O} & -2.10477 & -0.62182 & -1.36521 \\ \mathrm{H} & -2.75566 & -0.83871 & -2.03935 \\ \mathrm{H} & -2.27419 & 0.27894 & -1.04020 \\ \mathrm{O} & 1.03795 & -0.27310 & 1.48143 \\ \mathrm{H} & 1.03848 & 0.27480 & 2.27064 \\ \mathrm{H} & 0.21295 & -0.78824 & 1.47472 \\ \mathrm{O} & 0.53424 & 1.88838 & -0.51808 \\ \mathrm{H} & -0.35621 & 1.90089 & -0.12731 \\ \mathrm{H} & 0.65914 & 2.68361 & -1.04056 \\ \mathrm{O} & 0.60480 & -1.05073 & -1.27039 \\ \mathrm{H} & 0.97654 & -1.84190 & -1.66682 \\ \mathrm{H} & -0.31579 & -0.94663 & -1.56197 \\ \mathrm{O} & -1.58087 & -1.20499 & 1.27347 \\ \mathrm{H} & -1.81139 & -1.41746 & 0.35225 \\ \mathrm{H} & -2.00178 & -1.84768 & 1.85274\end{array}$

\begin{tabular}{lccl}
\multicolumn{5}{l}{ System $\mathrm{Li}+(\mathrm{H} 2 \mathrm{O}) 8$} \\
$\mathrm{Li}$ & -1.82502 & 0.24550 & 0.06123 \\
$\mathrm{O}$ & -1.17212 & 1.98792 & -0.27482 \\
$\mathrm{H}$ & -0.21196 & 2.10298 & -0.37488 \\
$\mathrm{H}$ & -1.60394 & 2.83956 & -0.36928 \\
$\mathrm{O}$ & -3.34128 & -0.58482 & -0.72079 \\
$\mathrm{H}$ & -4.29788 & -0.56352 & -0.79545 \\
$\mathrm{H}$ & -2.99221 & -1.24277 & -1.33117 \\
$\mathrm{O}$ & 1.58582 & 1.79346 & -0.35570
\end{tabular}




$\begin{array}{llll}\mathrm{H} & 2.18472 & 2.53761 & -0.47246 \\ \mathrm{H} & 1.82894 & 1.09731 & -1.00483 \\ \mathrm{O} & -0.61669 & -0.86177 & -0.95513 \\ \mathrm{H} & -0.02409 & -0.57886 & -1.66327 \\ \mathrm{H} & -0.09122 & -1.46667 & -0.40842 \\ \mathrm{O} & -1.30363 & -0.37420 & 1.78514 \\ \mathrm{H} & -0.42156 & -0.11283 & 2.09709 \\ \mathrm{H} & -1.81760 & -0.71147 & 2.52192 \\ \mathrm{O} & 2.02296 & -0.40910 & -1.83642 \\ \mathrm{H} & 2.16555 & -1.11301 & -1.17627 \\ \mathrm{H} & 2.58710 & -0.56374 & -2.59929 \\ \mathrm{O} & 1.61815 & -1.91832 & 0.37423 \\ \mathrm{H} & 1.65442 & -1.29024 & 1.11972 \\ \mathrm{H} & 1.87659 & -2.79095 & 0.68418 \\ \mathrm{O} & 1.36676 & 0.31073 & 1.94029 \\ \mathrm{H} & 1.48654 & 0.97099 & 1.22727 \\ \mathrm{H} & 1.87194 & 0.59792 & 2.70704\end{array}$

System $\mathrm{Li}+(\mathrm{H} 2 \mathrm{O}) 9$

$\begin{array}{llll}\mathrm{Li} & 1.81063 & -0.17593 & -0.51467 \\ \mathrm{O} & -2.41697 & 1.20970 & 0.60109 \\ \mathrm{H} & -1.95717 & 0.62948 & 1.24541 \\ \mathrm{H} & -3.24595 & 1.50107 & 0.99372 \\ \mathrm{O} & -1.25120 & -2.43009 & -0.00186 \\ \mathrm{H} & -1.73395 & -3.26083 & 0.04406 \\ \mathrm{H} & -1.67023 & -1.86395 & -0.68323 \\ \mathrm{O} & 1.45244 & -1.97724 & -0.08558 \\ \mathrm{H} & 2.12000 & -2.64952 & 0.06857 \\ \mathrm{H} & 0.57659 & -2.39143 & -0.13631 \\ \mathrm{O} & -2.19622 & -0.44514 & -1.58252 \\ \mathrm{H} & -2.46645 & 0.22942 & -0.92936 \\ \mathrm{H} & -2.82941 & -0.45602 & -2.30647 \\ \mathrm{O} & 3.65851 & 0.17335 & -0.20202 \\ \mathrm{H} & 3.67452 & 0.65771 & 0.63081 \\ \mathrm{H} & 4.52797 & 0.20588 & -0.60691 \\ \mathrm{O} & 1.39453 & 0.85517 & 1.09979 \\ \mathrm{H} & 0.75173 & 0.32990 & 1.59997 \\ \mathrm{H} & 0.94438 & 1.67950 & 0.85613 \\ \mathrm{O} & -0.26839 & 2.73677 & -0.17313 \\ \mathrm{H} & -0.23908 & 3.69106 & -0.28603 \\ \mathrm{H} & -1.16157 & 2.47315 & 0.10879 \\ \mathrm{O} & -0.91824 & -0.57182 & 1.99694 \\ \mathrm{H} & -0.98916 & -1.36760 & 1.43311 \\ \mathrm{H} & -1.01350 & -0.83934 & 2.91635 \\ \mathrm{O} & 0.47038 & 0.51007 & -1.66167 \\ \mathrm{H} & 0.22320 & 1.41267 & -1.40479 \\ \mathrm{H} & -0.34250 & 0.06054 & -1.92819\end{array}$




\begin{tabular}{lccc}
\multicolumn{4}{l}{ System Li+(H2O) 10} \\
Li & -0.77425 & 1.50249 & 0.19834 \\
$\mathrm{O}$ & 2.13359 & -0.27338 & 1.71103 \\
$\mathrm{H}$ & 2.53358 & 0.29577 & 1.02389 \\
$\mathrm{H}$ & 2.71654 & -0.28739 & 2.47607 \\
$\mathrm{O}$ & -2.63175 & 1.84814 & -0.03463 \\
$\mathrm{H}$ & -3.21978 & 2.36475 & 0.52048 \\
$\mathrm{H}$ & -3.12041 & 1.08455 & -0.38634 \\
$\mathrm{O}$ & 2.91800 & 1.01684 & -0.57849 \\
$\mathrm{H}$ & 2.64277 & 0.34100 & -1.23268 \\
$\mathrm{H}$ & 3.80646 & 1.31265 & -0.79887 \\
$\mathrm{O}$ & -3.22530 & -0.64564 & -0.96216 \\
$\mathrm{H}$ & -3.97529 & -1.05595 & -1.40017 \\
$\mathrm{H}$ & -2.88449 & -1.24090 & -0.26747 \\
$\mathrm{O}$ & -0.58874 & 0.55776 & 1.83895 \\
$\mathrm{H}$ & -1.09535 & -0.26385 & 1.76097 \\
$\mathrm{H}$ & 0.32963 & 0.32199 & 2.03008 \\
$\mathrm{O}$ & -0.61130 & 0.06007 & -1.09466 \\
$\mathrm{H}$ & 0.10878 & -0.12590 & -1.71213 \\
$\mathrm{H}$ & -1.45735 & -0.18121 & -1.50122 \\
$\mathrm{O}$ & 1.08536 & -2.33148 & 0.28003 \\
$\mathrm{H}$ & 1.40642 & -3.20875 & 0.51225 \\
$\mathrm{H}$ & 1.49053 & -1.68768 & 0.90024 \\
$\mathrm{O}$ & 0.73661 & 2.62692 & -0.03614 \\
$\mathrm{H}$ & 1.59067 & 2.25551 & -0.30931 \\
$\mathrm{H}$ & 0.79027 & 3.58494 & -0.04964 \\
$\mathrm{O}$ & 1.78752 & -0.99423 & -1.99045 \\
$\mathrm{H}$ & 2.06117 & -1.42124 & -2.80715 \\
$\mathrm{H}$ & 1.57502 & -1.67475 & -1.32048 \\
$\mathrm{O}$ & -1.65061 & -1.89474 & 0.87760 \\
$\mathrm{H}$ & -0.77975 & -2.01477 & 0.45515 \\
$\mathrm{H}$ & -1.82371 & -2.66827 & 1.42269
\end{tabular}

System $\mathrm{Li}+(\mathrm{H} 2 \mathrm{O}) 11$

$\begin{array}{cccc}\mathrm{Li} & 0.66273 & 1.65523 & -0.12095 \\ \mathrm{O} & 0.58544 & -2.31131 & -0.98703 \\ \mathrm{H} & -0.33176 & -2.57487 & -0.78320 \\ \mathrm{H} & 0.85283 & -2.74029 & -1.80563 \\ \mathrm{O} & -1.35863 & -0.74296 & 1.86204 \\ \mathrm{H} & -1.55886 & -1.14094 & 2.71480 \\ \mathrm{H} & -1.54070 & -1.40087 & 1.16357 \\ \mathrm{O} & 4.14802 & 0.19422 & -0.31785 \\ \mathrm{H} & 3.79485 & -0.61805 & 0.08020 \\ \mathrm{H} & 5.04255 & 0.33920 & -0.00186 \\ \mathrm{O} & -3.17243 & -0.12694 & -1.44023 \\ \mathrm{H} & -3.27452 & 0.51026 & -0.70524 \\ \mathrm{H} & -3.88115 & -0.00004 & -2.07674 \\ \mathrm{O} & -2.06562 & -2.32969 & -0.30787\end{array}$




$\begin{array}{rrrr}\mathrm{H} & -2.66430 & -3.07784 & -0.21966 \\ \mathrm{H} & -2.53141 & -1.62526 & -0.81117 \\ \mathrm{O} & -2.80843 & 1.34598 & 0.81507 \\ \mathrm{H} & -2.24151 & 0.67964 & 1.25833 \\ \mathrm{H} & -3.48781 & 1.61959 & 1.43919 \\ \mathrm{O} & 2.24302 & 1.90820 & -1.09337 \\ \mathrm{H} & 3.02467 & 1.37170 & -0.84820 \\ \mathrm{H} & 2.44078 & 2.39708 & -1.89392 \\ \mathrm{O} & -0.67975 & 2.97724 & 0.10036 \\ \mathrm{H} & -0.62732 & 3.88696 & 0.40070 \\ \mathrm{H} & -1.55160 & 2.61344 & 0.32545 \\ \mathrm{O} & 1.11941 & 0.55184 & 1.37151 \\ \mathrm{H} & 0.31848 & 0.07798 & 1.64386 \\ \mathrm{H} & 1.84621 & -0.08475 & 1.39840 \\ \mathrm{O} & -0.51866 & 0.27391 & -0.83049 \\ \mathrm{H} & 0.02925 & -0.50326 & -1.02339 \\ \mathrm{H} & -1.22564 & 0.31367 & -1.48682 \\ \mathrm{O} & 2.60384 & -1.78794 & 0.82858 \\ \mathrm{H} & 2.90271 & -2.52715 & 1.36495 \\ \mathrm{H} & 1.90639 & -2.10228 & 0.22355\end{array}$

System $\mathrm{Li}+(\mathrm{H} 2 \mathrm{O}) 12$

$\begin{array}{cccc}\mathrm{Li} & 0.09881 & 1.48357 & 0.08875 \\ \mathrm{O} & 2.43989 & -1.06673 & 1.81953 \\ \mathrm{H} & 2.80579 & -1.61755 & 2.51669 \\ \mathrm{H} & 1.98636 & -1.64036 & 1.14167 \\ \mathrm{O} & -0.14280 & 0.20164 & -1.31246 \\ \mathrm{H} & 0.66461 & 0.10645 & -1.84282 \\ \mathrm{H} & -0.92890 & 0.12161 & -1.86966 \\ \mathrm{O} & 1.21492 & -2.20883 & -0.16630 \\ \mathrm{H} & 1.62395 & -1.80159 & -0.94622 \\ \mathrm{H} & 0.26532 & -2.01396 & -0.21881 \\ \mathrm{O} & -3.57985 & 0.88175 & 0.32724 \\ \mathrm{H} & -4.48440 & 1.10789 & 0.56341 \\ \mathrm{H} & -3.21329 & 0.28689 & 1.01649 \\ \mathrm{O} & -1.43245 & 2.62602 & 0.13922 \\ \mathrm{H} & -2.30323 & 2.19646 & 0.16469 \\ \mathrm{H} & -1.45199 & 3.37662 & 0.73813 \\ \mathrm{O} & 0.21290 & 0.59652 & 1.75437 \\ \mathrm{H} & -0.57523 & 0.08712 & 1.99329 \\ \mathrm{H} & 0.99847 & 0.08774 & 2.01023 \\ \mathrm{O} & 1.74532 & 2.33356 & -0.31399 \\ \mathrm{H} & 2.59829 & 1.91896 & -0.09980 \\ \mathrm{H} & 1.90138 & 3.16120 & -0.77318 \\ \mathrm{O} & 3.76615 & 0.55923 & 0.14834 \\ \mathrm{H} & 3.42474 & -0.02039 & 0.86727 \\ \mathrm{H} & 4.70833 & 0.69313 & 0.28465 \\ \mathrm{O} & -1.62927 & -2.32284 & -0.30170\end{array}$




$\begin{array}{llll}\mathrm{H} & -2.02559 & -1.79384 & -1.02098 \\ \mathrm{H} & -1.76726 & -3.25582 & -0.49055 \\ \mathrm{O} & 2.43527 & -0.49622 & -2.03217 \\ \mathrm{H} & 2.91370 & -0.69982 & -2.84043 \\ \mathrm{H} & 3.04988 & -0.06997 & -1.40550 \\ \mathrm{O} & -2.75348 & -0.42054 & -1.93245 \\ \mathrm{H} & -3.29783 & -0.47523 & -2.72278 \\ \mathrm{H} & -3.21861 & 0.11858 & -1.26331 \\ \mathrm{O} & -2.20711 & -0.86759 & 1.87657 \\ \mathrm{H} & -2.52296 & -1.29829 & 2.67651 \\ \mathrm{H} & -2.00390 & -1.55416 & 1.20491\end{array}$

System $\mathrm{Li}+(\mathrm{H} 2 \mathrm{O}) 13$

$\begin{array}{cccc}\mathrm{Li} & 0.05615 & 2.04967 & -0.58212 \\ \mathrm{O} & 1.72005 & -1.72043 & -1.05746 \\ \mathrm{H} & 1.73520 & -2.00181 & -0.12975 \\ \mathrm{H} & 2.21578 & -0.88530 & -1.10860 \\ \mathrm{O} & -2.57063 & 1.07178 & 1.89963 \\ \mathrm{H} & -3.08547 & 1.21555 & 2.69947 \\ \mathrm{H} & -3.17991 & 0.79570 & 1.18441 \\ \mathrm{O} & 0.96114 & 0.78919 & 0.51053 \\ \mathrm{H} & 0.34512 & 0.06689 & 0.71764 \\ \mathrm{H} & 1.64521 & 0.81960 & 1.19112 \\ \mathrm{O} & -1.21881 & 0.78481 & -1.23357 \\ \mathrm{H} & -2.17431 & 0.81310 & -1.09262 \\ \mathrm{H} & -1.01571 & 0.01998 & -1.79578 \\ \mathrm{O} & 3.29009 & -0.00968 & 1.77185 \\ \mathrm{H} & 2.85319 & -0.87066 & 1.91794 \\ \mathrm{H} & 3.88330 & 0.17139 & 2.50569 \\ \mathrm{O} & 1.59192 & -2.15160 & 1.76248 \\ \mathrm{H} & 1.70356 & -3.00814 & 2.18477 \\ \mathrm{H} & 0.66404 & -1.85972 & 1.88371 \\ \mathrm{O} & 3.47648 & 0.50367 & -0.92744 \\ \mathrm{H} & 4.33338 & 0.35730 & -1.33857 \\ \mathrm{H} & 3.58222 & 0.40958 & 0.03839 \\ \mathrm{O} & 1.58803 & 2.42333 & -1.63498 \\ \mathrm{H} & 2.30774 & 1.79797 & -1.43208 \\ \mathrm{H} & 1.85901 & 2.99269 & -2.35697 \\ \mathrm{O} & -1.11950 & 3.03453 & 0.55414 \\ \mathrm{H} & -1.27449 & 3.95742 & 0.76303 \\ \mathrm{H} & -1.61461 & 2.47587 & 1.17734 \\ \mathrm{O} & -2.42597 & -2.24322 & -0.52197 \\ \mathrm{H} & -1.79527 & -2.18395 & -1.27865 \\ \mathrm{H} & -2.73500 & -3.15068 & -0.44715 \\ \mathrm{O} & -0.55481 & -1.69548 & -2.30997 \\ \mathrm{H} & -0.46877 & -1.98121 & -3.22340 \\ \mathrm{H} & 0.33996 & -1.75529 & -1.86978 \\ \mathrm{O} & -0.85704 & -1.09223 & 1.48549\end{array}$




$\begin{array}{llll}\mathrm{H} & -1.40423 & -0.37712 & 1.85625 \\ \mathrm{H} & -1.40542 & -1.55371 & 0.82602 \\ \mathrm{O} & -3.79167 & 0.08440 & -0.30496 \\ \mathrm{H} & -4.67805 & 0.20124 & -0.65784 \\ \mathrm{H} & -3.51910 & -0.84820 & -0.41842\end{array}$

System $\mathrm{Li}+(\mathrm{H} 2 \mathrm{O}) 14$

\begin{tabular}{llll}
$\mathrm{Li}$ & -0.02635 & -0.62604 & -0.23442 \\
$\mathrm{O}$ & 2.83580 & 1.71110 & -1.31184 \\
$\mathrm{H}$ & 3.24401 & 1.41571 & -0.47226 \\
$\mathrm{H}$ & 3.53447 & 2.02351 & -1.89402 \\
$\mathrm{O}$ & 0.37521 & 2.92290 & -1.24792 \\
$\mathrm{H}$ & 1.25515 & 2.49232 & -1.19003 \\
$\mathrm{H}$ & 0.42454 & 3.60195 & -1.92694 \\
$\mathrm{O}$ & -2.75079 & 1.25902 & 1.69203 \\
$\mathrm{H}$ & -2.09008 & 1.96254 & 1.51711 \\
$\mathrm{H}$ & -3.20205 & 1.46789 & 2.51527 \\
$\mathrm{O}$ & -1.35806 & -1.15322 & 1.21716 \\
$\mathrm{H}$ & -1.79340 & -0.36990 & 1.58629 \\
$\mathrm{H}$ & -2.05024 & -1.69562 & 0.80557 \\
$\mathrm{O}$ & -0.75575 & 3.03255 & 1.20177 \\
$\mathrm{H}$ & -0.39663 & 3.13714 & 0.29466 \\
$\mathrm{H}$ & -0.66264 & 3.86945 & 1.66509 \\
$\mathrm{O}$ & 3.62202 & 0.54461 & 1.01258 \\
$\mathrm{H}$ & 3.68441 & -0.43299 & 0.90460 \\
$\mathrm{H}$ & 4.25584 & 0.82357 & 1.67882 \\
$\mathrm{O}$ & -1.10189 & 0.54737 & -1.31347 \\
$\mathrm{H}$ & -0.74957 & 1.43844 & -1.45429 \\
$\mathrm{H}$ & -2.06902 & 0.57715 & -1.32637 \\
$\mathrm{O}$ & 1.63329 & -0.85795 & -1.37168 \\
$\mathrm{H}$ & 2.36957 & -1.35215 & -0.97896 \\
$\mathrm{H}$ & 1.98358 & 0.00662 & -1.63143 \\
$\mathrm{O}$ & -3.20473 & -2.23162 & -0.59351 \\
$\mathrm{H}$ & -4.58826 & 0.88671 & -1.01905 \\
$\mathrm{H}$ & -3.62992 & -1.36779 & -0.74777 \\
$\mathrm{H}$ & -3.88049 & -2.91477 & -0.57738 \\
$\mathrm{O}$ & 0.90629 & -2.72967 & 1.15638 \\
$\mathrm{H}$ & 0.43295 & -2.97444 & 0.34413 \\
$\mathrm{H}$ & 0.26758 & -2.21825 & 1.67334 \\
$\mathrm{O}$ & 0.85521 & 0.77182 & 0.87031 \\
$\mathrm{H}$ & 0.39771 & 1.46753 & 1.36023 \\
\hline & 1.77980 & 0.73984 & 1.15997 \\
\hline & -0.53396 & -2.40651 & -1.16190 \\
$\mathrm{H}$ & -1.48975 & -2.48323 & -1.30635 \\
$\mathrm{H}$ & -2.04477 & 0.51576
\end{tabular}




$\begin{array}{llll}\mathrm{H} & 2.48382 & -2.36360 & 0.89769 \\ \mathrm{H} & 3.97766 & -2.76505 & 0.53059\end{array}$

System $\mathrm{Li}+(\mathrm{H} 2 \mathrm{O}) 15$

\begin{tabular}{|c|c|c|c|}
\hline & 2.00027 & 1.75347 & -0.05091 \\
\hline & -0.28143 & 1.34858 & 0.74476 \\
\hline & -0.33383 & 0.46734 & 1.15894 \\
\hline & 0.30982 & 1.90922 & 1.27395 \\
\hline & 3.27798 & -0.61649 & -1.59167 \\
\hline & 3.09315 & -1.54666 & -1.36484 \\
\hline & 4.08911 & -0.58138 & -2.10634 \\
\hline & -2.06006 & 0.39114 & -1.37470 \\
\hline & -2.54466 & -0.43048 & -1.53296 \\
\hline & -1.28789 & 0.41263 & -1.95684 \\
\hline & 1.28074 & 3.28280 & -0.86885 \\
\hline & 1.87036 & 3.78134 & -1.43996 \\
\hline & 1.64613 & 3.28523 & 0.03577 \\
\hline & -1.43331 & 3.35142 & -0.87443 \\
\hline & -0.46084 & 3.43267 & -0.94302 \\
\hline & -1.84350 & 3.95965 & -1.49168 \\
\hline & -3.41871 & -1.58075 & 1.18522 \\
\hline & -4.04108 & -2.12716 & 1.67380 \\
\hline & -3.33924 & -1.94086 & 0.27810 \\
\hline & -0.65485 & -1.29202 & 1.43718 \\
\hline & -1.58921 & -1.39448 & 1.68224 \\
\hline & -0.57076 & -1.63756 & 0.52214 \\
\hline 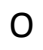 & -3.37673 & 1.18896 & 1.13681 \\
\hline & -3.97203 & 1.66190 & 1.72154 \\
\hline & -3.54996 & 0.23449 & 1.20915 \\
\hline ) & -2.76229 & -2.31486 & -1.35143 \\
\hline & -3.20760 & -2.88083 & -1.98764 \\
\hline & -1.79486 & -2.47334 & -1.39303 \\
\hline & -0.11 & -2.10172 & -1.08628 \\
\hline & 0.66916 & -2.66682 & -1.05232 \\
\hline & 0.18654 & -1.20368 & -1.37235 \\
\hline & 1.82199 & -2.07601 & 2.02813 \\
\hline & 0.86307 & -1.86316 & 2.05474 \\
\hline$y$ & 2.08974 & -2.42276 & 2.88389 \\
\hline & 1.93561 & 2.67172 & 1.67649 \\
\hline & 2.44806 & 1.83874 & 1.54590 \\
\hline $\mathrm{H}$ & 2.28042 & 3.15618 & 2.43009 \\
\hline & 2.43134 & -3.00455 & -0.46501 \\
\hline . & 2.31786 & -2.80853 & 0.48815 \\
\hline $\mathrm{H}$ & 2.86274 & -3.85966 & -0.55222 \\
\hline & 0.66730 & 0.36028 & -1.66900 \\
\hline 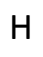 & 1.60517 & 0.33633 & -1.90771 \\
\hline$\Pi$ & 0.56885 & 0.94223 & -0.8911 \\
\hline & 92307 & 0.33123 & 1.02 \\
\hline
\end{tabular}




$\begin{array}{llll}\mathrm{H} & 2.61837 & -0.48936 & 1.44316 \\ \mathrm{H} & 3.16814 & 0.13053 & 0.10985\end{array}$

System $\mathrm{Li}+(\mathrm{H} 2 \mathrm{O}) 16$

\begin{tabular}{|c|c|c|c|}
\hline Li & 0.15607 & -0.04767 & -1.79818 \\
\hline $\mathrm{O}$ & -1.84491 & 2.71232 & -1.25457 \\
\hline $\mathrm{H}$ & -0.90552 & 2.97481 & -1.18076 \\
\hline H & -2.32919 & 3.44097 & -1.65351 \\
\hline 0 & 0.74977 & 2.69212 & 1.97329 \\
\hline $\mathrm{H}$ & 0.85137 & 3.30150 & 2.71003 \\
\hline & 1.47273 & 2.03498 & 2.00180 \\
\hline & 2.92567 & -2.50600 & -0.15930 \\
\hline 7 & 2.57256 & -2.42573 & 0.75062 \\
\hline $\mathrm{H}$ & 3.38877 & -3.34528 & -0.23732 \\
\hline & 1.63589 & 1.11926 & -2.15849 \\
\hline $\boldsymbol{H}$ & 2.50574 & 0.79245 & -1.88540 \\
\hline $\mathrm{H}$ & 1.47734 & 1.96343 & -1.70797 \\
\hline 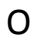 & -1.66705 & -3.29646 & -0.72833 \\
\hline $\mathrm{H}$ & -1.88725 & -4.22457 & -0.84647 \\
\hline $\mathrm{H}$ & -2.47596 & -2.76172 & -0.85594 \\
\hline O & -1.70088 & 0.12866 & -2.21855 \\
\hline $\mathrm{H}$ & -1.91675 & 1.04407 & -1.97117 \\
\hline $\mathrm{H}$ & -2.36532 & -0.43963 & -1.80479 \\
\hline 0 & 0.75763 & 3.36290 & -0.67629 \\
\hline $\mathrm{H}$ & 1.11834 & 4.24154 & -0.82886 \\
\hline $\mathrm{H}$ & 0.75105 & 3.20039 & 0.29070 \\
\hline 0 & -2.05420 & 2.00233 & 1.4510 \\
\hline $\mathrm{H}$ & -1.10621 & 2.04645 & 1.65348 \\
\hline $\mathrm{H}$ & -2.12440 & 2.23768 & 0.50920 \\
\hline $\mathrm{O}$ & -3.10400 & -0.33702 & 1.81510 \\
\hline $\mathrm{H}$ & -2.72705 & 0.59032 & 1.72409 \\
\hline $\mathrm{H}$ & -3.75553 & -0.32727 & 2.5221 \\
\hline 0 & 3.87499 & -0.01951 & -0.8375 \\
\hline $\mathrm{H}$ & 3.68942 & -0.97298 & -0.7387 \\
\hline $\mathrm{H}$ & 4.78705 & 0.08876 & $-1.1224 c$ \\
\hline $\mathrm{O}$ & 0.58771 & -1.88224 & -1.6120 \\
\hline $\mathrm{H}$ & -0.12977 & -2.51945 & -1.4797 \\
\hline $\mathrm{H}$ & 1.40120 & -2.23167 & -1.2215 \\
\hline $\mathrm{O}$ & 1.71032 & -1.89697 & 2.2015 \\
\hline $\mathrm{H}$ & 0.73879 & -1.88865 & 2.0297 \\
\hline $\mathrm{H}$ & 1.86156 & -2.30445 & 3.0593 \\
\hline $\mathrm{O}$ & 2.44294 & 0.66810 & 1.4176 \\
\hline $\mathrm{H}$ & 2.36759 & -0.19910 & 1.8511 \\
\hline $\mathrm{H}$ & 3.13424 & 0.60595 & 0.73510 \\
\hline $\mathrm{O}$ & -3.56282 & -1.37465 & -0.6568 \\
\hline $\mathrm{H}$ & -3.52499 & -0.97672 & 0.2406 \\
\hline $\mathrm{H}$ & -4.47682 & -1.36629 & -0.9555 \\
\hline $\mathrm{O}$ & -0.83719 & -1.78668 & 1.4218 \\
\hline
\end{tabular}




$\begin{array}{lrrr}\mathrm{H} & -1.66390 & -1.43486 & 1.80328 \\ \mathrm{H} & -1.07555 & -2.46114 & 0.75977 \\ \mathrm{O} & 0.00715 & 0.54610 & 0.06016 \\ \mathrm{H} & -0.39091 & -0.22317 & 0.50137 \\ \mathrm{H} & 0.90096 & 0.62424 & 0.43245\end{array}$

\begin{tabular}{|c|c|c|c|}
\hline \multicolumn{4}{|c|}{ System Li+(H2O)17 } \\
\hline $\mathrm{Li}$ & -0.56659 & -1.87817 & .14338 \\
\hline $\mathrm{O}$ & -2.56894 & -1.58441 & -2.16390 \\
\hline H & -2.90858 & -1.87419 & -3.01497 \\
\hline $\mathrm{H}$ & -1.87650 & -0.90150 & -2.31361 \\
\hline $\mathrm{O}$ & 0.94081 & 1.79074 & 1.78232 \\
\hline$H$ & 1.86176 & 1.53484 & 1.96259 \\
\hline H & 0.94666 & 2.55609 & 1.17937 \\
\hline $\mathrm{O}$ & -0.60561 & 0.23786 & -2.19178 \\
\hline $\mathrm{H}$ & -0.86174 & 1.17568 & -2.09334 \\
\hline $\mathrm{H}$ & 0.23397 & 0.19333 & -2.68617 \\
\hline $\mathrm{O}$ & 2.00416 & -0.03573 & -2.87368 \\
\hline $\mathrm{H}$ & 2.34394 & 0.60579 & -2.18799 \\
\hline $\mathrm{H}$ & 2.49787 & 0.10220 & -3.68678 \\
\hline 0 & -0.77047 & -2.90048 & -0.45030 \\
\hline $\mathrm{H}$ & -1.51406 & -2.71109 & -1.04047 \\
\hline $\mathrm{H}$ & 0.04512 & -2.8 & -0.97094 \\
\hline 0 & -1.39481 & 2.77648 & -1.49486 \\
\hline $\mathrm{H}$ & -1.97995 & 2.45232 & -0.74835 \\
\hline $\mathrm{H}$ & -1.91873 & 3.34638 & -2.06510 \\
\hline 0 & 0.88578 & 3.58702 & -0.30823 \\
\hline $\mathrm{H}$ & 0.04431 & 3.42043 & -0.78779 \\
\hline $\mathrm{H}$ & 1.05184 & 4.53366 & -0.29520 \\
\hline - & 2.74555 & 1.64074 & -1.03443 \\
\hline $\mathrm{H}$ & 2.16953 & 2.40417 & -0.86512 \\
\hline $\mathrm{H}$ & 3.06809 & 1.31006 & -0.17952 \\
\hline 0 & 2.85122 & -1.97989 & 0.93187 \\
\hline $\mathrm{H}$ & 3.53096 & -2.62887 & 0.72769 \\
\hline $\mathrm{H}$ & 2.26776 & -1.92015 & 0.14911 \\
\hline $\mathrm{O}$ & -4.03627 & -0.65958 & -0.06524 \\
\hline $\mathrm{H}$ & -3.65036 & -1.05549 & -0.87292 \\
\hline $\mathrm{H}$ & -4.98574 & -0.80794 & -0.06830 \\
\hline $\mathrm{O}$ & 3.40 & & 1.4 \\
\hline $\mathrm{H}$ & 4.26111 & 0.76613 & 1.90971 \\
\hline $\mathrm{H}$ & 3.26889 & -0.33700 & 1.37995 \\
\hline $\mathrm{O}$ & -2.78346 & 1.77603 & 0.42771 \\
\hline $\mathrm{H}$ & -3.30319 & 0.97320 & 0.25287 \\
\hline $\mathrm{H}$ & -2.40508 & 1.71432 & 1.32150 \\
\hline 0 & 1.73476 & -2.43396 & -1.61960 \\
\hline $\mathrm{H}$ & 1.86122 & -1.61453 & -2.14356 \\
\hline $\mathrm{H}$ & 2.13884 & -3.15919 & -2.10525 \\
\hline , & 0.65907 & -2.27918 & 2.5401 \\
\hline
\end{tabular}




$\begin{array}{llll}\mathrm{H} & 0.54846 & -2.86329 & 3.29329 \\ \mathrm{H} & 1.57817 & -2.31186 & 2.23027 \\ \mathrm{O} & -2.13306 & -1.18725 & 1.89757 \\ \mathrm{H} & -2.93658 & -1.09602 & 1.36396 \\ \mathrm{H} & -1.99911 & -0.36946 & 2.40217 \\ \mathrm{O} & 0.53684 & -0.51610 & 0.27083 \\ \mathrm{H} & 0.10088 & -0.27481 & -0.56613 \\ \mathrm{H} & 0.64434 & 0.30828 & 0.77777 \\ \mathrm{O} & -1.49191 & 1.35326 & 2.85125 \\ \mathrm{H} & -1.70293 & 1.75892 & 3.69641 \\ \mathrm{H} & -0.54777 & 1.51907 & 2.65036\end{array}$

System $\mathrm{Li}+(\mathrm{H} 2 \mathrm{O}) 18$

$\begin{array}{lrrr}\mathrm{Li} & -0.33170 & 0.31003 & -1.45657 \\ \mathrm{O} & -0.10881 & -2.55891 & 0.53459 \\ \mathrm{H} & 0.17128 & -1.98777 & -0.20697 \\ \mathrm{H} & -0.18312 & -1.97199 & 1.30827 \\ \mathrm{O} & -0.10224 & -1.14234 & 2.99969 \\ \mathrm{H} & 0.78126 & -0.72686 & 2.92295 \\ \mathrm{H} & -0.09284 & -1.70345 & 3.78082 \\ \mathrm{O} & 3.67970 & 0.85214 & -1.68293 \\ \mathrm{H} & 3.72660 & -0.12821 & -1.69541 \\ \mathrm{H} & 4.45129 & 1.20147 & -2.13713 \\ \mathrm{O} & 2.47153 & -0.19943 & 2.66138 \\ \mathrm{H} & 2.60083 & 0.56491 & 2.05782 \\ \mathrm{H} & 3.10186 & -0.12044 & 3.38336 \\ \mathrm{O} & 0.96873 & 1.60689 & -2.06126 \\ \mathrm{H} & 1.91335 & 1.40055 & -2.11293 \\ \mathrm{H} & 0.87220 & 2.44233 & -1.58320 \\ \mathrm{O} & 2.68011 & -2.41605 & 0.90430 \\ \mathrm{H} & 1.78115 & -2.77959 & 0.86489 \\ \mathrm{H} & 2.66830 & -1.69062 & 1.55037 \\ \mathrm{O} & 0.92799 & 3.65658 & -0.00566 \\ \mathrm{H} & 1.09589 & 4.59129 & -0.15652 \\ \mathrm{H} & 0.11762 & 3.57984 & 0.53676 \\ \mathrm{O} & 3.44489 & -1.82479 & -1.52080 \\ \mathrm{H} & 4.03035 & -2.48034 & -1.90959 \\ \mathrm{H} & 3.25844 & -2.08526 & -0.57741 \\ \mathrm{O} & -1.42168 & 3.24016 & 1.34095 \\ \mathrm{H} & -1.89206 & 3.93668 & 1.80658 \\ \mathrm{H} & -1.64163 & 2.38099 & 1.76203 \\ \mathrm{O} & 2.61748 & 1.63134 & 0.71793 \\ \mathrm{H} & 3.14791 & 1.41597 & -0.07242 \\ \mathrm{H} & 2.20527 & 2.50180 & 0.57169 \\ \mathrm{O} & -3.89003 & -0.83442 & 0.71160 \\ \mathrm{H} & -3.46988 & -1.66418 & 0.39178 \\ \mathrm{O} & -4.60079 & -1.07757 & 1.31289 \\ & -1.70963 & 1.71667 & -1.00957\end{array}$




$\begin{array}{llll}\mathrm{H} & -2.62441 & 1.55546 & -1.28734 \\ \mathrm{H} & -1.70661 & 2.48965 & -0.43083 \\ \mathrm{O} & -1.81536 & -0.89926 & -2.11931 \\ \mathrm{H} & -2.63475 & -0.41823 & -2.30905 \\ \mathrm{H} & -2.08560 & -1.70074 & -1.64331 \\ \mathrm{O} & -4.13169 & 0.56680 & -1.63736 \\ \mathrm{H} & -4.96707 & 0.80223 & -2.04855 \\ \mathrm{H} & -4.29674 & 0.11598 & -0.78881 \\ \mathrm{O} & -2.54714 & -2.94385 & -0.30903 \\ \mathrm{H} & -1.63453 & -2.99701 & 0.07807 \\ \mathrm{H} & -2.87039 & -3.82929 & -0.49544 \\ \mathrm{O} & 0.26896 & 0.15317 & 0.42124 \\ \mathrm{H} & 1.06878 & 0.70365 & 0.47069 \\ \mathrm{H} & -0.45224 & 0.58195 & 0.90944 \\ \mathrm{O} & -1.92025 & 0.70196 & 2.03768 \\ \mathrm{H} & -2.61850 & 0.23272 & 1.54671 \\ \mathrm{H} & -1.47755 & 0.06377 & 2.62345 \\ \mathrm{O} & 0.74857 & -1.40887 & -1.90356 \\ \mathrm{H} & 0.26560 & -1.71413 & -2.67873 \\ \mathrm{H} & 1.69674 & -1.59794 & -2.01427\end{array}$

System $\mathrm{Li}+(\mathrm{H} 2 \mathrm{O}) 19$

$\begin{array}{cccc}\mathrm{Li} & 0.01852 & 0.10467 & -1.80162 \\ \mathrm{O} & -0.02743 & 2.44782 & 1.12635 \\ \mathrm{H} & 0.43534 & 3.14654 & 0.62768 \\ \mathrm{H} & 0.04888 & 1.62722 & 0.58773 \\ \mathrm{O} & -0.34931 & 2.05396 & -1.81028 \\ \mathrm{H} & -1.23559 & 2.42740 & -1.91463 \\ \mathrm{H} & 0.29009 & 2.77570 & -1.73556 \\ \mathrm{O} & -2.70628 & 2.40674 & 1.11447 \\ \mathrm{H} & -3.13068 & 2.97055 & 1.76748 \\ \mathrm{H} & -1.73035 & 2.55350 & 1.16192 \\ \mathrm{O} & -1.98051 & -0.15161 & -1.87616 \\ \mathrm{H} & -2.53633 & 0.64162 & -1.91975 \\ \mathrm{H} & -2.56662 & -0.91836 & -1.84399 \\ \mathrm{O} & -1.45846 & -3.68881 & -0.71543 \\ \mathrm{H} & -2.33224 & -3.24277 & -0.71228 \\ \mathrm{H} & -1.59386 & -4.62290 & -0.89635 \\ \mathrm{O} & -3.61946 & -2.09154 & -0.65390 \\ \mathrm{H} & -4.55136 & -2.31645 & -0.71205 \\ \mathrm{H} & -3.48032 & -1.47229 & 0.09771 \\ \mathrm{O} & 0.81505 & 0.97516 & 3.17548 \\ \mathrm{H} & 0.52899 & 1.67736 & 2.54600 \\ \mathrm{H} & 1.14557 & 1.39338 & 3.97571 \\ \mathrm{O} & 0.33707 & -1.86875 & -1.89052 \\ \mathrm{H} & 1.22046 & -2.22358 & -2.06687 \\ \mathrm{H} & -0.27536 & -2.60215 & -1.74394 \\ \mathrm{O} & -0.14383 & -0.04086 & 0.25939\end{array}$




$\begin{array}{lrrr}\mathrm{H} & -1.09254 & -0.24747 & 0.20669 \\ \mathrm{H} & 0.30928 & -0.89002 & 0.38236 \\ \mathrm{O} & 3.44686 & 2.04894 & -0.36611 \\ \mathrm{H} & 3.27431 & 1.38956 & 0.35468 \\ \mathrm{H} & 4.39544 & 2.18921 & -0.42436 \\ \mathrm{O} & -2.80171 & -0.39162 & 1.21582 \\ \mathrm{H} & -2.30553 & -0.64860 & 2.01249 \\ \mathrm{H} & -2.93036 & 0.57261 & 1.23441 \\ \mathrm{O} & 0.26955 & -2.66579 & 1.18610 \\ \mathrm{H} & -0.21085 & -2.34449 & 1.96801 \\ \mathrm{H} & -0.35125 & -3.11828 & 0.58520 \\ \mathrm{O} & 2.91483 & 0.21636 & 1.44062 \\ \mathrm{H} & 2.20380 & 0.33725 & 2.08859 \\ \mathrm{H} & 2.97709 & -0.72512 & 1.21056 \\ \mathrm{O} & -0.94211 & -1.12248 & 3.15474 \\ \mathrm{H} & -1.14739 & -1.38775 & 4.05607 \\ \mathrm{H} & -0.31053 & -0.37188 & 3.19748 \\ \mathrm{O} & -3.09561 & 2.38346 & -1.59284 \\ \mathrm{H} & -3.79217 & 2.87985 & -2.02957 \\ \mathrm{H} & -3.15859 & 2.51229 & -0.62814 \\ \mathrm{O} & 3.07645 & -2.20673 & -1.86673 \\ \mathrm{H} & 3.19806 & -2.41262 & -0.92045 \\ \mathrm{H} & 3.75262 & -2.65464 & -2.38081 \\ \mathrm{O} & 1.94759 & 0.36117 & -2.06066 \\ \mathrm{H} & 2.49290 & 1.05644 & -1.66736 \\ \mathrm{H} & 2.48906 & -0.44116 & -2.09435 \\ \mathrm{O} & 2.89126 & -2.52969 & 0.83718 \\ \mathrm{H} & 3.41967 & -3.10036 & 1.40207 \\ \mathrm{H} & 1.93885 & -2.72154 & 1.00123 \\ \mathrm{O} & 1.49123 & 3.85135 & -0.66979 \\ \mathrm{H} & 1.71418 & 4.76704 & -0.85545 \\ \mathrm{H} & 2.32046 & 3.33438 & -0.57519\end{array}$

\begin{tabular}{llll}
\multicolumn{5}{l}{ System $\mathrm{Li}+(\mathrm{H} 2 \mathrm{O}) 20$} \\
$\mathrm{O}$ & -1.40132 & 1.64413 & -2.24857 \\
$\mathrm{H}$ & -1.01340 & 2.52107 & -2.11096 \\
$\mathrm{H}$ & -2.34088 & 1.64759 & -2.01104 \\
$\mathrm{O}$ & 0.28804 & 3.78800 & -1.69190 \\
$\mathrm{H}$ & 1.16586 & 3.38682 & -1.85070 \\
$\mathrm{H}$ & 0.27283 & 4.66214 & -2.09125 \\
$\mathrm{O}$ & 0.56950 & -3.58534 & -1.85018 \\
$\mathrm{H}$ & 0.60211 & -4.48517 & -2.18604 \\
$\mathrm{H}$ & 1.45640 & -3.18754 & -1.93659 \\
$\mathrm{O}$ & -3.91309 & 1.11408 & -1.16170 \\
$\mathrm{H}$ & -4.01945 & 0.13900 & -1.21161 \\
$\mathrm{H}$ & -4.76395 & 1.52622 & -1.33306 \\
$\mathrm{O}$ & -2.19865 & 1.39713 & 0.96944 \\
$\mathrm{H}$ & -2.47953 & 0.96886 & 1.79823
\end{tabular}




\begin{tabular}{|c|c|c|c|}
\hline & -2.92379 & 1.36875 & 0.32022 \\
\hline J & -2.74289 & -2.07196 & 1.28598 \\
\hline & -2.72374 & -1.45053 & 2.03367 \\
\hline & -1.82944 & -2.36828 & 1.13343 \\
\hline & -0.01655 & 0.13161 & -0.06928 \\
\hline & -0.76601 & 0.58722 & 0.36895 \\
\hline & 0.81162 & 0.57159 & 0.19197 \\
\hline & 2.55423 & 1.08386 & 0.52395 \\
\hline & 3.04899 & 0.25876 & 0.66769 \\
\hline & 2.46749 & 1.56771 & 1.37022 \\
\hline & -2.30857 & -0.11891 & 3.21499 \\
\hline & -2.71582 & -0.03579 & 4.08076 \\
\hline & -1.32740 & -0.07103 & 3.31644 \\
\hline & 1.73090 & -2.29101 & 2.45657 \\
\hline & 1.01307 & -2.51158 & 1.80431 \\
\hline & 1.75650 & -2.96130 & 3.14504 \\
\hline & 1.41215 & 0.03973 & -2.66429 \\
\hline & 2.00388 & -0.69402 & -2.44406 \\
\hline & 1.92007 & 0.86372 & -2.62053 \\
\hline & -1.30780 & -1.52904 & -2.17586 \\
\hline & -2.23295 & -1.67543 & -1.91816 \\
\hline & -0.85166 & -2.37496 & -2.27868 \\
\hline & 1.64448 & 2.43909 & 2.64434 \\
\hline & 1.96681 & 3.06569 & 3.29684 \\
\hline & 0.95081 & 2.87561 & 2.07884 \\
\hline & -3.82649 & -1.57475 & -1.02034 \\
\hline & -3.50033 & -1.76511 & -0.09602 \\
\hline & -4.54530 & -2.18046 & -1.22044 \\
\hline ) & -0.07588 & -2.44950 & 0.59210 \\
\hline & 0.05002 & -3.01469 & -0.18921 \\
\hline$y$ & -0.07 & -1.5 & 0.27747 \\
\hline o & -0.18557 & 3.24059 & 0.99936 \\
\hline & -0.03663 & 3.54608 & 0.08997 \\
\hline H & -1.01640 & 2.73286 & 1.02193 \\
\hline 0 & 3.65455 & -1.46790 & 0.81323 \\
\hline & 4.55992 & -1.59145 & 1.11373 \\
\hline $\mathrm{H}$ & 3.04999 & -1.84912 & 1.49391 \\
\hline 0 & 0.32420 & 0.05919 & 3.17697 \\
\hline 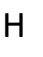 & 0.82102 & 0.89328 & 3.12184 \\
\hline $\mathrm{H}$ & 0.92372 & -0.68544 & 3.02091 \\
\hline 0 & 2.94426 & -2.16913 & -1.76330 \\
\hline $\mathrm{H}$ & 3.72176 & -2.42298 & -2.2696 \\
\hline $\mathrm{H}$ & 3.23077 & -1.97683 & -0.84693 \\
\hline 0 & 2.63715 & 2.39291 & -1.80133 \\
\hline $\mathrm{H}$ & 2.72275 & 1.98634 & -0.90856 \\
\hline $\mathrm{H}$ & 3.47857 & 2.78854 & -2.04452 \\
\hline 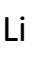 & -0.36598 & 0.09109 & -2.00657 \\
\hline
\end{tabular}




\section{REFERENCES}

(1) Frisch, M. J.; Trucks, G. W.; Schlegel, H. B.; Scuseria, G. E.; Robb, M. A.; Cheeseman, J. R.; Scalmani, G.; Barone, V.; Mennucci, B.; Petersson, G. A.; Nakatsuji, H.; Caricato, M.; Li, X.; Hratchian, H. P.; Izmaylov, A. F.; Bloino, J.; Zheng, G.; Sonnenberg, J. L.; Hada, M.; Ehara, M.; Toyota, K.; Fukuda, R.; Hasegawa, J.; Ishida, M.; Nakajima, T.; Honda, Y.; Kitao, O.; Nakai, H.; Vreven, T.; Montgomery, Jr., J. A.; Peralta, J. E.; Ogliaro, F.; Bearpark, M. J.; Heyd, J.; Brothers, E. N.; Kudin, K. N.; Staroverov, V. N.; Kobayashi, R.; Normand, J.; Raghavachari, K.; Rendell, A. P.; Burant, J. C.; Iyengar, S. S.; Tomasi, J.; Cossi, M.; Rega, N.; Millam, N. J.; Klene, M.; Knox, J. E.; Cross, J. B.; Bakken, V.; Adamo, C.; Jaramillo, J.; Gomperts, R.; Stratmann, R. E.; Yazyev, O.; Austin, A. J.; Cammi, R.; Pomelli, C.; Ochterski, J. W.; Martin, R. L.; Morokuma, K.; Zakrzewski, V. G.; Voth, G. A.; Salvador, P.; Dannenberg, J. J.; Dapprich, S.; Daniels, A. D.; Farkas, Ö.; Foresman, J. B.; Ortiz, J. V.; Cioslowski, J.; Fox, D. J. Gaussian, Inc.: Wallingford, CT, 2013.

(2) Becke, A. D. Density-functional exchange-energy approximation with correct asymptotic behavior. Phys. Rev. A 1988, 38, 3098-3100.

(3) Lee, C.; Yang, W.; Parr, R. G. Development of the Colle-Salvetti correlation-energy formula into a functional of the electron density. Phys. Rev. B 1988, 37, 785-789.

(4) (a) McLean, A. D.; Chandler, G. S. Contracted Gaussian-basis sets for molecular calculations. 1. 2nd row atoms, Z=11-18. J. Chem. Phys. 1980, 72, 5639-5648. (b) Raghavachari, K.; Binkley, J. S.; Seeger, R.; Pople, J. A. Self-Consistent Molecular Orbital Methods. 20. Basis set for correlated wave-functions. J. Chem. Phys. 1980, 72, 650-654.

(5) Zhao, Y.; Truhlar, D. G. The M06 suite of density functionals for main group thermochemistry, thermochemical kinetics, noncovalent interactions, excited states, and transition elements: two new functionals and systematic testing of four M06-class functionals and 12 other functionals. Theor. Chem. Acc. 2008, 120, 215-241.

(6) Lu, T.; Chen, F. Multiwfn: A Multifunctional Wavefunction Analyzer. J. Comput. Chem. 2012, 33, 580592.

(7) Hirshfeld F. L. Bonded-atom fragments for describing molecular charge densities. Theor. Chim. Acta 1977, 44, 129-138. 